Continuum Damping of Low- $n$

Toroidicity-Induced Shear Alfvén Eigenmodes

H.L. Berk, J.W. VAN DAM, Z. GUO, AND D.M. LINDBERG Institute for Fusion Studies

The University of Texas at Austin

Austin, Texas 78712

September 1991

\title{
THE UNIVERSITY OF TEXAS
}




\title{
Continuum Damping of Low- $n$ Toroidicity-Induced Shear Alfvén Eigenmodes
}

\author{
H.L. Berk, J.W. Van Dam, Z. Guo, and D.M. Lindberg \\ Institute for Fusion Studies \\ The University of Texas at Austin \\ Austin, Texas 78712
}

\begin{abstract}
The effect of resonant continuum damping is investigated for the low-mode-number, toroidicity-induced, global shear Alfvén eigenmodes, which can be self-excited by energetic circulating alpha particles in an ignited tokamak plasma. Resonant interaction with the shear Alfvén continuum is possible for these eigenmodes, especially near the plasma periphery, leading to significant dissipation, which is typically larger than direct bulk plasma dissipation rates. Two perturbation methods are developed for obtaining the Alfven resonance damping rate from the ideal fluid zeroth-order shear Alfvén eigenvalue and eigenfunction. In both methods the real part of the frequency is estimated to zeroth order, and the imaginary part, which includes the damping rate, is then obtained by perturbation theory. One method, which is applicable when the eigenfunction is nearly real, can readily be incorporated into general MHD codes. In the second method, the zeroth-order eigenfunctions may be complex; however, the application of this method to general MHD codes needs more detailed development. Also, an analytical estimate is found for the next-order real frequency shift of the fluid global Alfven mode. Analytical and numerical studies of this continuum damping effect in-
\end{abstract}


dicate that it can substantially reduce the alpha particle-induced growth rate. Thus, either it is possible to prevent instability or, if unstable, to use the Alfven resonance damping to estimate the saturation amplitude level predicted from quasilinear theory.

\section{DISCLAIMER}

This report was prepared as an account of work sponsored by an agency of the United States Government. Neither the United States Government nor any agency thereof, nor any of their Government. Neither the United States Governmes, or implied, or assumes any legal liability or responsiemployees, makecuracy, completeness, or usefulness of any information. apparatus, product, or bility for the accuracy, completeness, or usefulness of an wisclosed, or represents that its use would not infringe privately owned rights. Referprocess disclosed, or represents that if ence herein to any specific comrnercial product, process, or service by trade name, trademare manufacturer, or otherwise does not necessarily constitute or imply its endors mendation, or favoring by the United States Government of and opinions of authors expressed herein do 


\section{Introduction}

Of considerable attention in the last few years has been the study of the effect of highenergy alpha particles on the stability of shear Alfvén waves in ignited tokamak plasmas. In particular, it has been shown that a global-type low-mode-number shear Alfvén mode that is created by toroidicity ${ }^{1}$ can be strongly destabilized by fusion-product alpha particles. ${ }^{2,3}$ (For short, we shall refer to this toroidicity-induced shear Alfvén eigenmode as the TAE mode.) Marginal stability thresholds in the alpha particle beta value (with bulk plasma Landau damping taken into account) and growth rates above threshold have been calculated ${ }^{4-9}$ for typical reactor parameters. with the threshold beta values found to be fairly low. Moreover, guiding-center simulation ${ }^{10}$ have indicated that finite-amplitude TAE waves may cause loss of containment of fast alpha particles, which would be detrimental to the self-sustainment of a burning plasma by means of alpha particle heating. Apropos of this issue, the saturation amplitude level for the TAE mode has been studied ${ }^{11}$ with a quasilinear profile broadening theory based on wave trapping. Experimentally, it may be possible to simulate the TAE mode with the use of highly energetic circulating ions injected by tangentially-directed neutral beams, and some recently reported results ${ }^{12-14}$ seem to indicate that this mode has been observed.

In the present paper. we extend the linear stability studies of the low-mode-number TAE mode by investigating an additional physical mechanism. namely. the stabilizing effect of resonance wave damping that is caused by interaction of the TAE mode with the shear Alfvén continuum. Such continuum resonance is likely to occur near the edge of the plasma where the plasma density decreases and the safety factor increases. We find that the continuum resonance leads to dissipation that can be more important than the bulk Landau damping from the toroidal plasma ion and electron species. Furthermore, we find that this continuum damping may be able to reduce the alpha particle-induced growth rate significantly and thus have an ameliorating effect on the TAE instability. 
Our theoretical procedures for obtaining the continuum damping rate are as follows. In one method, the variational nature of the governing equations is used to obtain the dissipative contributions. We find that the continuum damping rate can be predicted from the ideal MHD equations if we use the prescription of causality; this is the same prescription that is used in the derivation of Landau damping to handle wave-particle resonance in velocity space, although here we apply it to the shear Alfvén resonance in coordinate space. The application of causality to analyze spatial continuum resonance in the ideal MHD equations has been employed before, for specific equilibrium profiles, to derive the power absorption rate due to wave heating. ${ }^{15-21}$ In our TAE problem, we exploit the fact that the continuum dissipation rate is generally small compared to the oscillation frequency of the basic TAE mode. Then, as long as it can be treated as a perturbation. the continuum damping rate can be obtained in terms of the zeroth-order TAE eigenfunction and its real eigenfrequency. The variational method depends on the assumption that the eigenfunction is nearly real. However, if continuum resonances occur at several spatial points, this assumption may fail. To treat this latter case we have developed an alternate perturbation method to estimate damping, where the zeroth-order eigenfunction may be complex. Recently, a related theory has been developed for continuum damping of the high-mode-number version of the TAE mode. ${ }^{22}$

In Sec. II, we briefly review the physics of the TAE mode and how it is destabilized by alpha particles. The TAE coupled wave equation will be derived in the large-aspect-ratio limit in Sec. III. A general formalism for obtaining the damping rate due to continuum resonance will be described in Sec. IV. Explicit formulae for the case of two-mode coupling and for the case of three-mode coupling are given in Sec. V, along with an analytic estimate for the frequency shift of the MHD global Alfven wave. Numerical solutions are presented in Sec. VI and compared with the analytical estimates. Section VII contains comments and conclusions concerning this work. Several appendices are provided, including a derivation for 
the kinetic response of the alpha particles and an analytical estimate for the TAE growth rate induced by the alpha particles, as well as a description of an alternate method of obtaining the damping rate when several continuum singularities exist for real frequency.

\section{Review of the TAE Instability}

In a tokamak plasma there are two types of shear Alfvén waves that are global in nature, with extended radial structure. Both have low mode numbers, and the derivative along a magnetic field line of their respective wave functions does not vanish on any characteristic flux surface. The first type, called the "global Alfvén eigenmode," is a regular wave (due to the inclusion of the Hall effect) with a discrete frequency that lies just below the minimum of the Alfvén continuum. ${ }^{23-26}$ Early theoretical analysis of this mode in cylindrical geometry showed that transit wave-particle resonant interaction with super-Alfvénic alpha particles could destabilize it, although somewhat weakly. ${ }^{27}$ Subsequent analysis in toroidal geometry found that finite toroidicity causes coupling among modes with different poloidal mode numbers, which. when Landau damping is accounted for, has the tendency to lead to complete stabilization of this mode. ${ }^{5.28}$

The other type of global shear Alfvén wave exists only in toroidal geometry. Its discrete frequency lies within "gaps" in the shear Alfvén continuum that are created by toroidal coupling effects. ${ }^{29-32}$ The existence of this wave. the TAE mode, was originally shown in the ideal MHD limit, without any alpha particles. ${ }^{1}$ In a burning tokamak plasma, this TAE mode can be strongly destabilized by circulating, highly energetic alpha particles through inverse Landau wave-particle resonant interaction. ${ }^{2-9}$ The high-mode-number version of this mode has also been examined. ${ }^{33,34}$ In the present work, we confine our attention to the low-modenumber eigenmode, whose radially global mode structure can be diagnosed experimentally.

The "gaps" in the toroidal Alfvén continuum are caused by toroidicity-induced mode coupling, as illustrated in Fig. 1(a). This figure shows the toroidal shear Alfvén continuum 
(solid curves), as well as the cylindrical continua (dashed curves) for the poloidal harmonics with mode numbers $m=1$ and $m=2$, where the toroidal mode number is taken to be $n=1$. The perturbed wavefunction is assumed to vary as $\exp [i(-m \theta+n \zeta-\omega t)]$. For simplicity, in Fig. 1(a) the density profile is taken to be constant, and the safety factor profile is quadratic; i.e., $q(r)=1+(r / a)^{2}$, with $q=2$ at the edge of the plasma $r=a$. The cylindrical continua are given by the frequencies $\omega=\omega(r)$ that satisfy the shear Alfven condition $\omega^{2}=k_{\| m, n}^{2}(r) v_{A}^{2}(r)$. Here $k_{\| m, n}=[-m / q(r)+n] / R$ is the parallel wavenumber, with $q(r)$ the safety factor, $n$ and $m$ the toroidal and poloidal mode numbers, $R$ the major radius, and $v_{A}(r)=B\left(4 \pi N_{i} M_{i}\right)^{-1 / 2}$ the Alfvén speed. Due to the tokamak axisymmetry, the toroidal mode number $n$ is a good "quantum" number, and we will henceforth suppress this index on $k_{\|}$and on the wavefunction. However, the poloidal mode numbers are no longer good "quantum" numbers in tokamak geometry, due to toroidicity-induced coupling. In particular, at the radial location $r=r_{g}$ where two cylindrical continua cross; i.e., $k_{\| m}=-k_{\| m+1}$, the small toroidicity effects resolve the degeneracy between the two cylindrical continua, and a "gap" appears. For the case shown in Fig. 1(a), viz., $n=1$ and $m=1$ and 2 , this crossing point occurs where $q=1.5$. In the general case, if the cylindrical-geometry Alfvén continua corresponding to modes $m$ and $m+1$ (for toroidal mode number $n$ ) intersect, this occurs where $q=(m+1 / 2) / n$. The magnitude of the width of the gap, $\Delta \omega$. is on the order of the inverse aspect ratio $a / R$.

The continuum Alfvén modes correspond to the excitation of shear Alfvén waves on a given flux surface where the mode frequency is resonant, viz., where $\omega^{2}=k_{\| m}^{2}(r) v_{A}^{2}(r)$. From causality arguments one can show that such resonance leads to wave absorption. However, frequencies excited within the spectral gaps, are not resonant with the continuum and hence will not damp in the gap region. This allows a discrete eigenfrequency of the toroidicityinduced Alfvén eigenmode (TAE) to be established.

The TAE mode structure that corresponds to the situation illustrated in Fig. 1(a) is 
shown in Fig. 1(b) for $a / R=0.25$. Its eigenfrequency $\omega_{0}=0.93\left|k_{\|} v_{A}\right|_{q=1.5}$ lies within the continuum gap of Fig. 1(a). Note that the mode structure has a sharp transition for the $m=1$ and $m=2$ components at the gap location.

The destabilizing effects of alpha particles on the TAE modes have been studied perturbatively. To a good approximation since the beta value of the alpha particles of small $\left(\beta_{\alpha} \ll \varepsilon\right)$, one can assume that the imaginary part of the frequency is small compared to the real part when the inverse Landau transit resonance contribution of the fast alpha particles is included. The growth rate can then be obtained from the quadratic form for the coupled equations, evaluated with the MHD eigenfunction and eigenfrequency for the TAE mode. More detailed discussions of this procedure and results for the alpha particle-induced growth rate may be found in Refs. 2-9 and in Appendix A of the present paper. This appendix includes. with more general derivation, an additional kinetic response due to the finite alpha particle banana width in the equilibrium distribution function, as was also described in Ref. 11.

Actually, the features of the shear Alfvén spectrum in toroidal geometry are somewhat more complicated than what has been described in Fig. 1. Much of the initial work on TAE instability with alpha particles was limited to simple cases for which the value of the safety factor at the plasma edge was specified to be $q(a) \approx 2$, as in Fig. 1, and for which a constant density profile was assumed. For a radially decreasing density profile, the Alfvén speed is increased, especially near the plasma edge. It is then possible for the TAE eigenmode frequency $\omega_{0}$ to intersect with the shear Alfvén continuum at the radial location $r_{s}$ where the continuum resonance condition $\omega_{0}^{2}=k_{\| m}^{2}\left(r_{s}\right) v_{A}^{2}\left(r_{s}\right)$ is satisfied. Figure 2 illustrates such a case. In this figure, $q(a)=2.3$ and the density is constant out to $r / a=0.9$ and then decreases linearly to zero at the edge. Continuum resonance occurs near the plasma periphery. It is known from earlier theoretical studies ${ }^{16-20}$ of Alfvén wave heating that continuum resonance leads to wave damping. Resonance can also occur due to the radial variation of the safety 
factor within the plasma.

Furthermore, as the value of $q(a)$ increases, more than a single gap can occur in the toroidal spectrum. Figure 3 shows the case for which $q(a)=3$. Coupling between the $m=1$ and $m=2$ modes occurs in the region where $q=1.5$, and coupling between the $m=2$ and $m=3$ modes occurs in the region where $q=2.5$. For the case shown in Fig. 3, the gaps at $q=1.5$ and 2.5 approximately "line up," and the eigenmode structure and resonant dissipation are more complicated. When the density and safety factor profiles lead to multiple gaps that do not line up, the TAE mode that is established in the lowest-order gap (i.e., with the lowest frequency and located nearest the magnetic axis) can interact with the Alfvén continuum at interior radial locations which also leads to significant damping.

The details concerning the nature of the TAE mode and the dissipative effect of resonant interaction with the toroidal shear Alfvén continuum will be explained in the following sections of this paper. In the next several sections, the appropriate system of coupled eigenmode equations will be derived, and the wave damping that is caused by the resonance of the TAE mode with the shear Alfvén continuum will be calculated. The casual reader may wish to skip directly to the description of the numerical results (Sec. VI) and the concluding discussion (Sec. VII).

\section{Eigenmode Equations in the Large Aspect Ratio Limit}

The governing equations for shear Alfvén waves have been previously derived in cylindrical geometry. ${ }^{35-37}$ Later work included the kinetic effects of alpha particles on shear Alfvén wave stability. ${ }^{27,38.39}$ The corresponding equations have also been derived in toroidal geometry, both in the large-aspect-ratio limit for use with model profiles ${ }^{2,3}$ and also in general geometry for use with numerically generated equilibria. ${ }^{1}$ Since the limit of large aspect ratio toroidal geometry preserves all the essential features of the TAE mode, while at the same time 
facilitates a clear understanding of the important physics mechanisms, we will adopt that approach in the present work. The previous large-aspect ratio derivation of the eigenmode equations $^{2,3}$ was restricted to the case of concentric circular flux surfaces and retained only the toroidal coupling that enters through the frequency-dependent ion polarization inertial term. Here we allow non-concentric flux surfaces and include also the toroidal coupling in the magnetic field line-bending term.

\section{A. Low-Beta Shear Alfvén Wave Equation}

The basic equations from which we begin our derivation of the shear Alfvén eigenmode equations are the following: (i) the condition for charge neutrality,

$$
\nabla \cdot \mathbf{J}=0
$$

where $\mathbf{J}$ is the current, given by Ampére's law (with the neglect of the displacement current) as $4 \pi \mathrm{J} / c=\nabla \times \mathrm{B}$; (ii) the momentum balance equation,

$$
N_{i} M_{i} \frac{d \mathbf{v}}{d t}=\frac{1}{c}(\mathbf{J} \times \mathbf{B})-\nabla \cdot \mathbf{P}
$$

where $\mathrm{v}$ is the macroscopic plasma velocity and where the total pressure $\mathrm{P}=p_{c} \mid+\mathrm{P}_{\alpha}$ results from the isotropic core plasma pressure $p_{c}$ and the possibly anisotropic alpha-particle pressure $P_{\alpha}$ : and (iii) the drift kinetic equation,

$$
\frac{\partial f}{\partial t}+\left(v_{\|} \hat{\mathbf{b}}+\mathbf{v}_{E}+\mathbf{v}_{D}\right) \cdot \nabla f+\frac{e}{M}\left(v_{\|} E_{\|}+\mathbf{v}_{D} \cdot \mathbf{E}\right) \frac{\partial f}{\partial \mathcal{E}}=0
$$

which describes the kinetic behavior of the highly energetic alpha particle population, as well as the Landau damping due to the plasma ions and electrons. In Eq. (3), $f$ is the distribution function for the respective species; $\epsilon$ and $M$ are the particle charge and mass: $\mathbf{v}_{E}=c(\mathbf{E} \times \mathbf{B}) / B^{2}$ is the electric field drift velocity; and $\mathbf{v}_{D}=\hat{\mathrm{b}} \times\left(\mu \nabla B+v_{\|}^{2} \boldsymbol{\kappa}\right) / \omega_{c}+v_{\|}^{2} \hat{\mathrm{b}} \hat{\mathrm{b}}$. $\nabla \hat{\mathrm{b}} / \omega_{c}$ is the magnetic field gradient and curvature drift velocity, with $\hat{\mathrm{b}}=\mathbf{B} / B$ a unit vector along the magnetic field direction, $\mu=v_{\perp}^{2} / 2 B$ the magnetic moment per unit mass, 
$\mathcal{E}=v_{\|}^{2} / 2+\mu B$ the particle energy per unit mass, $\boldsymbol{\kappa}=(\hat{\mathbf{b}} \cdot \nabla) \hat{\mathbf{b}}$ the field line curvature, and $\omega_{c}=e B / M c$ the gyrofrequency.

We now linearize the field variables as $\mathbf{J}=\mathbf{J}_{0}+\delta \mathbf{J}, \mathbf{B}=\mathbf{B}_{0}+\delta \mathbf{B}, \mathbf{E}=\delta \mathbf{E}$, and $\mathbf{v}=\delta \mathbf{v}$, where the subscript designates the steady-state equilibrium quantities, with small perturbations $|\delta \mathbf{B}| /\left|\mathbf{B}_{0}\right| \ll 1$, etc. The density perturbation may be ignored since we employ charge neutrality, and equilibrium electrostatic potential effects are neglected. Perturbed quantities will be taken to vary in time as $\exp (-i \omega t)$. The electric field and the magnetic field may be represented in terms of the scalar and vector potentials $\Phi$ and $\mathbf{A}$ as

$$
\delta \mathbf{E}=-\nabla \delta \Phi-\frac{1}{c} \frac{\partial}{\partial t} \delta \mathbf{A}, \quad \delta \mathbf{B}=\nabla \times \delta \mathbf{A}
$$

For the shear Alfvén problem, the low-beta approximation is valid, and in this limit we may take $\delta \mathbf{A} \cong \delta A_{\|} \widehat{\mathbf{b}}$, set $\delta B_{\|} \cong 0$ since $\delta B_{\|}$is related to the high-frequency compressional Alfvén branch, and drop terms involving the equilibrium pressure. Also, since the TAE mode is essentially an MHD wave, we may set $\delta E_{\|}=0$ and thus obtain the relationship

$$
i \omega \delta A_{\|}=c \hat{\mathrm{b}} \cdot \nabla \delta \Phi
$$

We also assume that the fluid velocity $\delta \mathbf{v}$ is mainly given by the perturbed $\mathbf{E} \times \mathbf{B}$ motion:

$$
\delta \mathbf{v} \cong \delta \mathbf{v}_{E}=\frac{c(\delta \mathbf{E} \times \mathbf{B})}{B^{2}}
$$

Henceforth, except where required to avoid confusion, the subscript on equilibrium quantities will be dropped.

Using Eq. (2) to obtain the perpendicular perturbed current $\delta \mathbf{J}_{\perp}$ and setting $\mathbf{B} \cdot \delta \mathbf{B}=0$, we can rewrite Eq. (1) in the following form:

$(\mathbf{B} \cdot \nabla)\left(\frac{\mathbf{B} \cdot \delta \mathbf{J}}{B^{2}}\right)-i \omega c \nabla \cdot\left(\frac{N_{i} M_{i} \mathbf{B} \times \delta \mathbf{v}}{B^{2}}\right)+\left(\delta \mathbf{B}_{\perp} \cdot \nabla\right)\left(\frac{\mathbf{B} \cdot \mathbf{J}}{B^{2}}\right)+c \nabla \cdot\left[\frac{\mathbf{B}}{B^{2}} \times(\nabla \cdot \delta \mathbf{P})\right]=0$.

With the use of the expression $\mathbf{v}_{D}=\left(v_{\|} / \omega_{c}\right) \nabla \times\left(\widehat{\mathbf{b}} v_{\|}\right)$, where the spatial gradient $\nabla$ is here understood to operate with $\mathcal{E}$ and $\mu$ held fixed, we find the following equation, valid in the 
limit of low beta and large aspect ratio (see Appendix A):

$$
c \nabla \cdot\left[\frac{\mathbf{B}}{B^{2}} \times(\nabla \cdot \delta \mathrm{P})\right] \cong \sum_{s} e \int d^{3} v \mathbf{v}_{D} \cdot \nabla \delta f,
$$

where $\sum_{s}$ indicates a sum over species. Equation (8) permits the evaluation of the last term in Eq. ( 7 ) by means of the solution of the drift kinetic equation, Eq. (3). Now, with the use of Eqs. (6) and (8), we can rewrite Eq. (7) as

$$
(\mathbf{B} \cdot \nabla)\left(\frac{\mathbf{B} \cdot \delta \mathbf{J}}{B^{2}}\right)+\left(\delta \mathbf{B}_{\perp} \cdot \nabla\right)\left(\frac{\mathbf{B} \cdot \mathbf{J}}{B^{2}}\right)+\frac{i \omega c^{2}}{4 \pi} \nabla \cdot\left(\frac{1}{v_{A}^{2}} \nabla_{\perp} \delta \Phi\right)+\sum_{s} e \int d^{3} v \mathbf{v}_{D} \cdot \nabla \delta f=0
$$

The form of Eq. (9) is nearly identical to that of the shear Alfven equation derived by Rosenbluth and Rutherford ${ }^{27}$ in their study of fast particle destabilization of kinetic Alfvén waves, apart from terms involving gradients acting on the equilibrium magnetic field strength $B$. which were ignored in their cylindrical treatment.

Now examine the various individual terms in Eq. (9). For $\delta \mathbf{A}=\delta A_{\|} \hat{\mathrm{b}}$, we can express the parallel perturbed current, which appears in the first term of Eq. (9), as

$$
\frac{4 \pi}{c}(\mathbf{B} \cdot \delta \mathbf{J})=-\nabla \cdot\left[B^{2} \nabla_{\perp}\left(\frac{\delta A_{\|}}{B}\right)\right]+\frac{\delta A_{\|}}{B}\left\{4 \pi \nabla \nabla \cdot \mathrm{P}+\left[\frac{4 \pi \mathbf{B} \times(\nabla \cdot \mathrm{P})}{B^{2}}\right]+\left(\frac{4 \pi}{c} J_{\|}\right)^{2}\right\} .
$$

Note that in Eq. (10) all the derivatives acting on the perturbed field quantity are contained in the first term. Also, the second and third terms are is negligible in the low-beta limit, and the fourth term is dropped, being of order of the square of the inverse aspect ratio. Next we find that the perpendicular perturbed magnetic field. which appears in the second term of Eq. (9), can be expressed as

$$
\delta \mathbf{B}_{\perp}=-\mathbf{B} \times \nabla\left(\frac{\delta A_{\|}}{B}\right)+4 \pi\left(\frac{\mathbf{B} \times \nabla \cdot \mathbf{P}}{B^{2}}\right)\left(\frac{\delta A_{\|}}{B}\right)
$$

where the second term is negligible in the low-beta limit. Next, recall that the equilibrium parallel current (also known as the Pfirsch-Schlüter, or return, current) in an axisymmetric toroidal system, which appears in the third term in Eq. (9), is given by

$$
\left(\frac{4 \pi}{c}\right) J_{\|} \cong \frac{B}{I}\left[R^{2} \nabla \cdot\left(\frac{1}{R^{2}} \nabla \psi\right)\right]
$$


where $I=R B_{T}$ is a function only of the poloidal flux $\Psi$ in the low-beta limit, with $B_{T}$ the toroidal magnetic field and $R$ the major radius (measured from the axis of toroidal symmetry). The approximation in Eq. (12) again follows froin the assumption of low beta. The procedure for obtaining the perturbed distribution function, which appears in the last term of Eq. (9), from the drift kinetic equation, Eq. (3), is described in Appendix A.

Introducing Eqs. (10)-(12) into Eq. (9), we obtain the eigenmode equation

$$
\begin{aligned}
-(\mathbf{\mathbf { B }} \cdot \nabla) & \left\{\frac{1}{B^{2}} \nabla \cdot\left[B^{2} \nabla_{\perp}\left(\frac{1}{B^{2}}(\mathbf{B} \cdot \nabla) \delta \Phi\right)\right]\right\}-\left[\mathbf{B} \times \nabla\left(\frac{1}{B^{2}}(\mathbf{B} \cdot \nabla) \delta \Phi\right)\right] \\
\cdot & \nabla\left[\frac{R^{2}}{I} \nabla \cdot\left(\frac{1}{R^{2}} \nabla \Psi\right)\right] \\
& -\nabla \cdot\left(\frac{\omega^{2}}{v_{A}^{2}} \nabla_{\perp} \delta \Phi\right)+\left(\frac{4 \pi i \omega}{c^{2}}\right) \sum_{s} e \int d^{3} v \mathbf{v}_{D} \cdot \nabla \delta f=0 .
\end{aligned}
$$

Except for the approximation of low beta. Eq. (13) describes shear Alfvén waves in general axisymmetric toroidal geometry.

It is convenient to construct a quadratic form from Eq. (13), as follows. Multiply Eq. (13) by $\left(c^{2} / 8 \pi \omega\right) \delta \Phi^{*}$ and integrate over all space, with the assumption of no contribution from the surface terms. In this way, we obtain the equation

$$
Q(\omega, \delta \Phi)=-\frac{i}{2} \sum_{s} \epsilon_{s} \int d^{3} r \int d^{3} v \delta f \mathbf{v}_{D} \cdot \delta \mathbf{F}_{\perp}^{*}
$$

with the functional $Q(\omega, \delta \Phi)$ defined by

$$
Q(\omega, \delta \Phi)=-\omega(\delta W-\delta I)
$$

where $\delta W$ is the ideal MHD potential energy in the zero-beta limit and $\delta I$ is the inertial energy of the perturbation:

$$
\begin{gathered}
\delta W=\frac{c^{2}}{8 \pi \omega^{2}} \int d^{3} r\left\{\left|B \nabla_{\perp}\left(\frac{\mathbf{B} \cdot \nabla \delta \Phi}{B^{2}}\right)\right|^{2}+\delta \Phi^{*}\left[\mathbf{B} \times \nabla_{\perp}\left(\frac{\mathbf{B} \cdot \nabla \delta \Phi}{B^{2}}\right)\right] \cdot \nabla\left[\frac{R^{2}}{I} \nabla \cdot\left(\frac{\nabla \psi}{R^{2}}\right)\right]\right\} \\
\delta I=\frac{c^{2}}{8 \pi} \int d^{3} r \frac{1}{v_{A}^{2}}\left|\nabla_{\perp} \delta \Phi\right|^{2}
\end{gathered}
$$


The term on the right-hand side of Eq. (21a), obtained by integration by parts, is proportional to the power transfer from the alpha particles to the electromagnetic field:

$$
\sum_{s} e_{s} \int d^{3} r \int d^{3} v \delta f \mathbf{v}_{D} \cdot \delta \mathbf{E}_{\perp}^{*}=\int d^{3} r\left(\mathbf{J} \cdot \delta \mathbf{E}_{\perp}^{*}\right)
$$

The inertial energy $\delta I$ is clearly positive definite, and if we assume ideal MHD stability, then $\delta W$ is also positive definite for any bounded perturbation $\delta \Phi$.

If we take the kinetic term on the right-hand side of Eq. (14) to be small, then to lowest order the eigensolution is $\delta \Phi_{0}$, with frequency $\omega_{0}$, and it satisfies $Q\left(\omega_{0}, \delta \Phi_{0}\right)=0$. To next order, the shift in the eigenvalue, $\delta \omega=\omega-\omega_{0}$, due to the kinetic term treated as a perturbation is given by the equation

$$
i \delta \omega \frac{\partial Q\left(\omega_{0}, \delta \Phi_{0}\right)}{\partial \omega_{0}}=\frac{1}{2} \sum_{s} e_{s} \int d^{3} r \int d^{3} v \delta f v_{D} \cdot \delta E_{\perp}^{*}
$$

Equation (19) can be exactly interpreted as the time rate of change of the wave energy (lefthand side) being equal to the power transfer of alpha particles to the electromagnetic field (right-hand side). Furthermore, the wave energy can be easily seen to be positive:

$$
\frac{\partial Q}{\partial \omega}=-\frac{\partial}{\partial \omega}\left[\frac{1}{\omega}\left(\omega^{2} \delta W\right)-\delta I\right]=\delta W+\delta I>0
$$

by virtue of the assumed MHD stability. (Later we will point out that this conclusion depends on the perturbation $\delta \dot{\varphi}$ not being singular in the main domain of integration.)

\section{B. Large-Aspect-Ratio Eigenmode Equation}

For the sake of simplicity, we now reduce the general equation for the TAE mode to its form in the limit of large aspect ratio. Assume that the inverse aspect ratio $\varepsilon=a / R_{0}$ is small relative to unity, where $a$ is the minor radius at the plasma edge and $R_{0}$ is the major radius as measured to the location of the magnetic axis. Then, we introduce the flux-type large-aspect-ratio coordinates $(r, \theta, \zeta)$, where $r$ is the minor radius coordinate (centered on the magnetic axis), $\theta$ is a poloidal angle coordinate, and $\zeta$ is the toroidal angle coordinate. 
These coordinates ${ }^{40,41}$ are related to the cylindrical coordinates $(R, \phi, Z)$ centered on the toroidal axis of symmetry as follows:

$$
\begin{aligned}
& R=R_{0}+r \cos \theta-\Delta(r)+r \eta(r)(\cos 2 \theta-1) \\
& \phi=-\zeta \\
& Z=r \sin \theta+r \eta(r) \sin 2 \theta .
\end{aligned}
$$

The Shafranov shift of the flux surfaces is given by $\Delta(r)$; here the convention is $\Delta>0$, since the inward nature of the shift for flux surfaces with increasing minor radius has been accounted for with an explicit negative sign in Eq. (21a). The quantity $\eta(r)=\left(r / R_{0}+\Delta^{\prime}\right) / 2$, with $\Delta^{\prime}=d(\Delta) / d r$, is small, of $\mathcal{O}(\varepsilon)$. Solving the Grad-Shafranov equilibrium equation by means of an expansion in powers of $\epsilon$ yields $\Delta^{\prime}=\left(r / R_{0}\right)\left(\beta_{p}+\ell_{i} / 2\right)$, with $\beta_{p}=2(\langle p\rangle-p) / B_{p}^{2}$ a measure of the poloidal beta value and $\ell_{i}=\left\langle B_{p}^{2}\right\rangle / B_{p}^{2}$ the internal inductance, where the brackets $\langle\cdots\rangle$ indicate a volume average. Note that the shift $\Delta$ is $\mathcal{O}\left(\varepsilon^{2}\right)$, whereas its radial derivative, $\Delta^{\prime}$, is $\mathcal{O}(\varepsilon)$.

In the absence of the terms that involve the quantity $\eta(r)$, the coordinates $(r, \theta, \zeta)$ in Eq. (21) would be identical to the well-known Shafranov coordinates ${ }^{42}$ (which were used in Ref. 1). However, in the Shafranov coordinates representation, the safety factor $q=$ B $\cdot \nabla \zeta / \mathbf{B} \cdot \nabla \theta$ would contain $\theta$-variation to $\mathcal{O}(\varepsilon)$. Since our intention is to investigate the effects of toroidicity-induced poloidal coupling to $\mathcal{O}(\varepsilon)$, it is more convenient for our purpose to employ the flux-type large-aspect-ratio coordinates defined in Eq. (21), for which $q=$ $q(r)+\mathcal{O}\left(\varepsilon^{2}\right)$, where $r$ is itself a flux coordinate to $\mathcal{O}\left(\varepsilon^{2}\right)$. With these flux-type coordinates, the derivative operator along a field line, $\mathbf{B} \cdot \nabla$, involves poloidal dependence only in the overall multiplicative factor $\mathrm{B} \cdot \nabla \theta$, which is related to the Jacobian for these coordinates; i.e., $\mathbf{B} \cdot \nabla \delta \Phi=(\mathbf{B} \cdot \nabla \theta)[\partial / \partial \theta+q(r) \partial / \partial \zeta] \delta \Phi(r, \theta, \zeta)$. 
With these large-aspect-ratio coordinates, we have the following representations:

$$
\begin{aligned}
& \mathbf{B}=\frac{r B_{0}}{q(r)} \nabla r \times \nabla(q \theta-\zeta) \\
& \mathbf{B} \cdot \nabla \theta=\left(\frac{r B_{0}}{q(r)}\right) \frac{1}{J} \\
& J=r R_{0}\left(1+\frac{2 r}{R_{0}} \cos \theta\right)+\mathcal{O}\left(\varepsilon^{2}\right) . \\
& g^{r r}=\nabla r \cdot \nabla r=1+2 \Delta^{\prime} \cos \theta, \quad g^{\theta \theta}=\nabla \theta \cdot \nabla \theta=\frac{1}{r^{2}}\left[1-2\left(\frac{r}{R_{0}}+\Delta^{\prime}\right) \cos \theta\right] \\
& \nabla r \cdot \nabla \theta=-\frac{1}{r} \sin \theta\left[\frac{r}{R_{0}}+\left(r \Delta^{\prime}\right)^{\prime}\right], \quad \nabla \zeta \cdot \nabla \zeta=\frac{1}{R_{0}^{2}}\left[1-2\left(\frac{r}{R_{0}}\right) \cos \theta\right]
\end{aligned}
$$

with $\nabla r \cdot \nabla \zeta=\nabla \theta \cdot \nabla \zeta=0$. The various other metric coefficients for this coordinate system can be worked out straightforwardly. The quantity $J=[\nabla r \cdot(\nabla \theta \times \nabla \zeta)]^{-1}$ is the Jacobian.

We now examine the form of the individual terms in Eq. (13) in this large-aspect-ratio tokamak equilibrium. Noting that

$\nabla \cdot\left(B^{2} \nabla_{\perp} \Psi\right)=-\nabla \cdot[\mathbf{B} \times(\mathbf{B} \times \nabla \Psi)]=\frac{1}{J} \frac{\partial}{\partial r}\left(B^{2} J g^{r r} \frac{\partial \Psi}{\partial r}\right)+\frac{1}{J} \frac{\partial}{\partial \theta}\left(B^{2} J g^{\theta \theta} \frac{\partial \Psi}{\partial \theta}\right)+\mathcal{O}\left(\varepsilon^{2}\right)$

we can write the Laplacian-like first term in Eq. (13) exactly, to $\mathcal{O}\left(\varepsilon^{2}\right)$, as follows:

$$
\begin{gathered}
(\mathbf{B} \cdot \nabla)\left\{\frac{1}{B^{2}} \nabla \cdot\left[B^{2} \nabla_{\perp}\left(\frac{1}{B^{2}}(\mathbf{B} \cdot \nabla) \delta \Phi\right)\right]\right\}=(\mathbf{B} \cdot \nabla)\left\{\frac{1}{J B^{2}} \frac{\partial}{\partial r}\left[J B^{2} g^{r r} \frac{\partial}{\partial r}\left(\frac{1}{B^{2}}(\mathbf{B} \cdot \nabla) \delta \Phi\right)\right]\right. \\
\left.+\frac{1}{J B^{2}} \frac{\partial}{\partial r}\left[J B^{2} g^{\theta \theta} \frac{\partial}{\partial \theta}\left(\frac{1}{B^{2}}(\mathbf{B} \cdot \nabla) \delta \Phi\right)\right]\right\} .
\end{gathered}
$$

For the most part, the $\mathcal{O}(\varepsilon)$ terms that arise from toroidicity-induced coupling contribute only small modifications to the Alfvén wave behavior. However, the $\mathcal{O}(\varepsilon)$ toroidicity contributions become quite significant in those terms of the eigenmode equation that involve a double derivative of the wave function $\delta \Phi$ with respect to radius, because the coefficient of $\partial^{2} \delta \Phi / \partial r^{2}$ almost vanishes when the mode frequency $\omega$ is nearly resonant with the shear 
Alfvén continuum. In particular, the $\mathcal{O}(\varepsilon)$ terms are able to resolve the degeneracy that occurs when the cylindrical spectra for two different poloidal harmonics cross each other: i.e., when $k_{\| m}^{2} v_{A}^{2}=k_{\| m^{\prime}}^{2} v_{A}^{2}$ occurs, for modes with poloidal mode numbers $m$ and $m^{\prime}$, at some radial location $r_{m, m^{\prime}}$. When this happens, the $\mathcal{O}(\varepsilon)$ terms give rise to a "gap" in the toroidal spectrum of singular frequencies, whose gap width is $\mathcal{O}(\varepsilon)$. The TAE mode exists within such gaps. With this discussion in mind, we will retain the toroidal $\mathcal{O}(\varepsilon)$ coupling effects only in those terms that involve a second-order radial derivative of the wave functicn.

We now proceed to multiply each term in Eq. (13) by the factor $J / r R_{0}$, where $J$ is the Jacobian given in Eq. (22c); to Fourier transform the wavefunction as

$$
\delta \Phi(r, \theta, \zeta)=\sum_{m} \Phi_{m}(r) \exp (-i m \theta+i n \zeta)
$$

where the toroidal mode number $n$ is conserved: to define the local quantity

$$
k_{\| m}=\left(-\frac{m}{q(r)}+n\right) \frac{1}{R_{0}}
$$

which is obviously the parallel wavelength in the cylindrical limit; to introduce the new wave function

$$
E_{m}(r)=\frac{1}{r} \Phi_{m}(r)
$$

and to move the parallel gradient operators in between the two radial derivative operators in the first term of Eq. (13). Incidentally, note the useful identity

$$
\frac{1}{r} \frac{d}{d r}\left[r g(r) \frac{d \Phi_{m}}{d r}\right]=\frac{1}{r^{2}} \frac{d}{d r}\left[r^{2} g(r) \frac{d E_{m}}{d r}\right]+\left(\frac{d g}{d r}\right) E_{m}+\frac{1}{r} g(r) E_{m}
$$

for an arbitrary radial function $g(r)$. In this way we manipulate the first term of Eq. (13) to find that

$$
\begin{aligned}
& -\left(\frac{J}{r R_{0}}\right)(\mathbf{B} \cdot \nabla)\left\{\frac{1}{B^{2}} \nabla \cdot\left[B^{2} \nabla_{\perp}\left(\frac{1}{B^{2}}(\mathbf{B} \cdot \nabla) \delta \Phi\right)\right]\right\} \\
& \quad \Rightarrow \frac{1}{r^{2}} \frac{d}{d r}\left[r^{3} k_{\| m}^{2} \frac{d E_{m}}{d r}\right]+\frac{k_{\| m} E_{m}}{r^{2}} \frac{d}{d r}\left(r^{3} \frac{d k_{\| m}}{d r}\right)-\left(\frac{m^{2}-1}{r}\right) k_{\| m}^{2} E_{m} \\
& \quad+\frac{1}{r^{2}} \frac{d}{d r}\left[r^{3} \Delta^{\prime} k_{\| m}\left(k_{\| m+1} \frac{d E_{m+1}}{d r}+k_{\| m-1} \frac{d E_{m-1}}{d r}\right)\right]
\end{aligned}
$$


Next, consider the second term of Eq. (13). Since it does not entail a double radial derivative of the wave function, we evaluate it without toroidicity corrections as follows:

$$
-\left(\frac{J}{r R_{0}}\right)\left[\mathbf{B} \times \nabla\left(\frac{1}{B^{2}}(\mathbf{B} \cdot \nabla) \delta \Phi\right)\right] \cdot \dot{\nabla}\left[\frac{R^{2}}{I(\Psi)} \nabla \cdot\left(\frac{1}{R^{2}} \nabla \psi\right)\right] \Rightarrow-\frac{k_{\| m} E_{m}}{r^{2}} \frac{d}{d r}\left(r^{2} \frac{d k_{\| m}}{d r}\right) \text {. }
$$

This second term exactly cancels part of the first term of Eq. (13).

Next, evaluate the third term of Eq. (13). Consistent with our earlier argument, we retain the toroidal corrections to $\mathcal{O}(\varepsilon)$ in the portion of this term that involves a second-order radial derivative of the wave function and thus obtain

$$
\begin{aligned}
& -\left(\frac{J}{r R_{0}}\right) \nabla \cdot\left(\frac{\omega^{2}}{v_{A}^{2}} \nabla_{\perp} \delta \Phi\right) \Rightarrow-\frac{1}{r^{2}} \frac{d}{d r}\left[r^{3}\left(\frac{\omega}{v_{A}}\right)^{2} \frac{d E_{m}}{d r}\right]-E_{m} \frac{d}{d r}\left(\frac{\omega}{v_{A}}\right)^{2} \\
& +\left(\frac{m^{2}-1}{r}\right)\left(\frac{\omega}{v_{A}}\right)^{2} E_{m}-\frac{1}{r^{2}} \frac{d}{d r}\left[r^{3}\left(\frac{\omega}{v_{A}}\right)^{2}\left(\Delta^{\prime}+\frac{2 r}{R_{0}}\right)\left(\frac{d E_{m+1}}{d r}+\frac{d E_{m-1}}{d r}\right)\right]
\end{aligned}
$$

where $v_{A 0}=B_{0} /\left[N_{i}(r) M_{i}\right]^{1 / 2}$ contains radial variation only through the density $N_{i}(r)$, the constant $B_{0}$ being the magnetic field strength evaluated at the magnetic axis.

Since the fourth term of Eq. (13), viz., the kinetic effects for alpha particle-induced destabilization, etc., can be treated as a perturbation, we neglect toroidal corrections to this term. Moreover, since it does not essentially influence the continuum resonance problem. we will simply represent this term in the following symbolic fashion:

$$
\frac{-4 \pi i \omega r^{2}}{c^{2}} \sum_{s} e \int d^{3} v \mathbf{v}_{D} \cdot \nabla \delta f \Longrightarrow i L_{k}(\omega) E_{m}
$$

Here $L_{k}(\omega)$ is a kinetic operator, perturbative in nature, that represents alpha-particle instability drive, various plasma dissipation effects, and possibly other non-ideal MHD effects.

Finally, collecting the results given in Eqs. (29)-(32), we obtain the TAE wave equation, including toroidicity effects to lowest order:

$$
\frac{d}{d r}\left[r^{3}\left(\frac{\omega^{2}}{v_{A}^{2}}-k_{\| m}^{2}\right) \frac{d E_{m}}{d r}\right]+r^{2} E_{m} \frac{d}{d r}\left(\frac{\omega}{v_{A}}\right)^{2}-\left(m^{2}-1\right)\left(\frac{\omega^{2}}{v_{A}^{2}}-k_{\| m}^{2}\right) r E_{m}+i L_{k}(\omega) E_{m}
$$


$+\frac{d}{d r}\left[r^{3}\left(\frac{\omega}{v_{A}^{2}}\right)^{2}\left(\Delta^{\prime}+\frac{2 r}{R_{0}}\right)\left(\frac{d E_{m+1}}{d r}+\frac{d E_{m-1}}{d r}\right)-r^{3} \Delta^{\prime} k_{\| m}\left(k_{\| m+1} \frac{d E_{m+1}}{d r}+k_{\| m-1} \frac{d E_{m-1}}{d r}\right)\right]=0$

The last terms of Eq. (33), which involve $r / R_{0}$ and $\Delta^{\prime}$, are $\mathcal{O}(\varepsilon)$ and comprise the toroidal coupling. Because the poloidal mode number may assume any integer values, Eq. (33) actually constitutes a system of equations that couple the mode amplitudes $E_{m}$.

Before ending this section, we introduce two simplifications of Eq. (33). First, we observe that the terms involving $\dot{r} / R_{0}$ and $\Delta^{\prime}$ are only small corrections except in the gap regions where there occurs a degeneracy of the form $k_{\| m} \cong-k_{\| m \pm 1}$, with $k_{\| m}=\omega / v_{A}$. Since the toroidal coupling is important only in the vicinity of such gaps, we may make the simplification:

$$
\left[\left(\frac{v^{2}}{v_{A}^{2}}\right)\left(\Delta^{\prime}+\frac{2 r}{R_{0}}\right)-\Delta^{\prime}\left(k_{\| m} k_{\| m \pm 1}\right)\right]\left(\frac{d E_{m \pm 1}}{d r}\right) \cong 2\left(\Delta^{\prime}+\frac{r}{R_{0}}\right)\left(\frac{\omega^{2}}{v_{A}^{2}}\right)\left(\frac{d E_{m \pm 1}}{d r}\right) .
$$

Next, we will approximate the quantity $\Delta^{\prime}=\left(r / R_{0}\right)\left(\beta_{p}+\ell_{i} / 2\right)$ by the constant value it assumes for a plasma with zero beta and constant current profile, namely, $\Delta^{\prime}=r / 4 R_{0}$. This approximation is justified to the extent that the validity of our large aspect ratio reducedMHD treatment requires $\Delta^{\prime}$ to remain small.

Using Eq. (34) and the approximated form of $\Delta^{\prime}$, we can now simplify Eq. (33) to the following form:

$$
\begin{gathered}
\frac{d}{d r}\left[r^{3}\left(\frac{\omega^{2}}{v_{A}^{2}}-k_{\| m}^{2}\right) \frac{d E_{m}}{d r}\right]+\frac{d}{d r}\left(\frac{\omega}{v_{A}}\right)^{2} r^{2} E_{m}-\left(m^{2}-1\right)\left(\frac{\omega^{2}}{v_{A}^{2}}-k_{\| m}^{2}\right) r E_{m} \\
+\frac{d}{d r}\left[r^{3} \widehat{\varepsilon}(r)\left(\frac{\omega^{2}}{v_{A}^{2}}\right)\left(\frac{d E_{m+1}}{d r}+\frac{d E_{m-1}}{d r}\right)\right]=-i L_{k}(\omega) E_{m}
\end{gathered}
$$

where we have defined the coupling quantity $\widehat{\varepsilon}(r)=5 r / 2 R_{0}$. Equation (35) is identical to the eigenmode equation given in Refs. 2 and 3, except that the coupling strength of $5 r / 2 R_{0}$ obtained in the present work is more accurate, compared to $3 r / 2 R_{0}$ in the earlier work. The reasons for the difference are that Refs. 2 and 3 assumed concentric circular equilibrium flux surfaces and also neglected the coupling from the field line bending. 
In the remainder of this paper, we will solve the coupled system of equations represented in Eq. (35), both numerically and analytically.

\section{Vriational Formalism for Shear Alfvén Continuum Damping Rate}

In this section we develop a perturbation method for obtaining the Alfvén damping rate within the ideal fluid equation context from the zeroth-order TAE eigenfunction and its real eigenvalue, by applying an analytic continuation technique based on the prescription of causality. We shall first use the cylindrical approximation to describe damping due to the shear Alfvén continuum resonance and then treat the general coupled-mode problem in toroidal geometry. This method is accurate if the eigenfunction is nearly real.

\section{A. Formalism in Cylindrical Geometry}

In cylindrical geometry, the poloidal modes are uncoupled and, from Eq. (35), the reduced MHD equation for the amplitude of the $m^{\text {th }}$ harmonic takes the form

$\frac{d}{d r}\left[r^{3}\left(\frac{\omega^{2}}{v_{A}^{2}}-k_{\| j m}^{2}\right) \frac{d E_{m}}{d r}\right]+\omega^{2} r^{2} E_{m} \frac{d}{d r}\left(\frac{1}{v_{A}^{2}}\right)-\left(m^{2}-1\right)\left(\frac{\omega^{2}}{v_{A}^{2}}-k_{\| m}^{2}\right) r E_{m}=-i L_{k}(\omega) E_{m}$.

In Eq. (36), $L_{k}(\omega)$ is a perturbation operator that primarily represents the kinetic instability drive due to high-energy alpha particles, as well as the bulk plasma Landau damping and perhaps other non-ideal MHD dissipation. For definiteness, we solve Eq. (36) with the boundary condition $E_{m}(a)=0$ at the plasma boundary $r=a$.

From Eq. (36) we can construct a quadratic form by multiplying by $E_{m}(r)$ and integrating between two arbitrary radial points $r_{1}$ and $r_{2}$, taking care to retain the endpoint contributions:

$$
\int_{r_{1}}^{r_{2}} d r r\left\{\left[r^{2}\left(\frac{d E_{m}}{d r}\right)^{2}+\left(m^{2}-1\right) E_{m}^{2}\right]\left(\frac{\omega^{2}}{v_{A}^{2}}-k_{\| m}^{2}\right)-\omega^{2} r E_{m}^{2} \frac{d}{d r}\left(\frac{1}{v_{A}^{2}}\right)\right\}
$$




$$
=i \int_{r_{1}}^{r_{2}} d r E_{m} L_{k}(\omega) E_{m}+\left.\left(E_{m} C_{m}\right)\right|_{r_{1}} ^{r_{2}}
$$

In Eq. (3i), the "flux" $C_{m}(r)$ is given by

$$
C_{m}(r)=\left(\frac{\omega^{2}}{v_{A}^{2}}-k_{\| m}^{2}\right) r^{3} \frac{d E_{m}(r)}{d r}
$$

and it is assumed that the quantity $\left[\left(\omega / v_{A}\right)^{2}-k_{\| m}^{2}\right]$ does not vanish anywhere in the interval from $r_{1}$ to $r_{2}$. Should there exist within this interval a point $r_{s}$ or points $r_{s, j}$ where the equality $\left(\omega / v_{A}\right)^{2}-k_{\| m}^{2}=0$ holds, then Eq. (37) is modified to the following exact relationship:

$$
\begin{aligned}
& \mathcal{P} \int_{r_{1}}^{r_{2}} r d r\left\{\left[r^{2}\left(\frac{d E_{m}}{d r}\right)^{2}+\left(m^{2}-1\right) E_{m}^{2}\right]\left(\frac{\omega^{2}}{v_{A}^{2}}-k_{\| m}^{2}\right)-\omega^{2} r E_{m}^{2} \frac{d}{d r}\left(\frac{1}{v_{A}^{2}}\right)\right\} \\
= & i \int_{r_{1}}^{r_{2}} d r E_{m} L_{\alpha}(\omega) E_{m}+\left.\left(E_{m} C_{m}\right)\right|_{r_{1}} ^{r_{2}}+\sum_{j}\left[E_{m}\left(r_{s, j}^{-}\right) C_{m}\left(r_{s, j}^{-}\right)-E_{m}\left(r_{s, j}^{+}\right) C_{m}\left(r_{s, j}^{+}\right)\right]
\end{aligned}
$$

where

$$
\mathcal{P} \int_{r_{1}}^{r_{2}}=\lim _{\delta \rightarrow 0}\left(\int_{r_{1}}^{r_{5}^{-}}+\int_{r_{s}^{+}}^{r_{2}}\right)
$$

denotes the principal value integral, with $r_{s}^{ \pm}=r_{s} \pm \delta$, and we use the appropriate generalization to the principal value integral when multiple singular points $r_{s, j}$ occur. We shall take $C_{m}(r)$ to be continuous at the singular points $r_{s, j}$. Letting $r_{1} \rightarrow 0$ and $r_{2} \rightarrow a$, we finally. obtain the equation

$$
G\left(\omega, E_{m}\right)=-\sum_{j}\left[E_{m}\left(r_{s, j}^{+}\right)-E_{m}\left(r_{s, j}^{-}\right)\right] C_{m}\left(r_{s, j}\right)+i \int_{0}^{a} d r E_{m} L_{k}(\omega) E_{m}
$$

where the quantity $G\left(\omega, E_{m}\right)$ is defined by

$$
\begin{aligned}
G\left(\omega, E_{m}\right) \equiv & \lim _{\rho \rightarrow 0} \mathcal{P} \int_{\rho}^{a} d r r\left\{\left[r^{2}\left(\frac{d E_{m}}{d r}\right)^{2}+\left(m^{2}-1\right) E_{m}^{2}\right]\left(\frac{\omega^{2}}{v_{A}^{2}}-k_{\| m}^{2}\right)-\omega^{2} r E_{m}^{2} \frac{d}{d r}\left(\frac{1}{v_{A}^{2}}\right)\right\} \\
& +\delta_{m, 0}\left(\frac{\omega^{2}}{v_{A}^{2}(0)}-k_{\| m}^{2}(0)\right) \lim _{\rho \rightarrow 0} \rho^{3} E_{m}(\rho) \frac{d E_{m}(\rho)}{d \rho} .
\end{aligned}
$$

The principle value in Eq. (42) is necessary since $d E_{m} / d r \propto\left(\omega^{2} / v_{A}^{2}-k_{\| m}^{2}\right)^{-1}$ as $r \rightarrow r_{s, j}$. Note that the quantity $G\left(\omega, E_{m}\right)$ defined in Eq. (42) is related to the quadratic form $Q(\omega, \delta \Phi)$ 
of Eq. (14) as $G\left(\omega, E_{m}\right)=\omega Q(\omega, \delta \Phi)$. Also, for simplicity, we will ignore from now on the endpoint contribution at the origin for $m=0$.

We wish to treat the two terms on the right-hand side of Eq. (41), namely, the $L_{k}$ kinetic term and the singular wave function behavior at $r_{s, j}$, in a perturbative manner. Thus, to lowest order, we will pose the problem such that $L_{k}$ is neglected and the wavefunction is taken to be continuous at $r_{s, j}$ : i.e., $E_{m}\left(r_{s, j}^{+}\right)=E_{m}\left(r_{s, j}^{-}\right)$. The lowest-order solution $E_{m}^{(0)}$, with both $C_{m}^{(0)}(r)$ and $E_{m}^{(0)}(r)$ continuous everywhere, satisfies $G\left(\omega_{0}, E_{m}^{(0)}\right)=0$, which will allow for a real eigenfrequency $\omega_{0}$. Then, to next order, we shall find an expression for the complex shift in the eigenfrequency. The discontinuity of the next-order wavefunction at the points $r_{s, 3}$ can be obtained by means of the application of the Landau causality procedure to a logarithmically divergent structure, as will be shown in the discussion to follow.

Our formal perturbation treatment assumes that the terms on the right-hand side of Eq. (41) are small and expands the eigenfunction and eigenfrequency as

$$
E_{m}(r)=E_{m}^{(0)}(r)+\delta E_{m}(r), \quad \omega=\omega_{0}+\delta \omega
$$

It can be shown that Eq. (41) without the $L_{\alpha}$ term, solved with regular boundary conditions at the origin $r=0$ and the boundary condition $E_{m}(a)=0$ at the edge and with $E_{m}(r)$ and $C_{m}(r)$ continuous across the points $r_{s, j}$, admits solutions with real eigenvalues $\omega_{0}$ and real eigenfunctions $E_{m}^{(0)}(r)$, which satisfy $G\left(\omega_{0}, E_{m}^{(0)}\right)=0$. This constitutes the lowest-order solution.

Now consider the effect of the perturbation, which enters in next order. At the origin and at the plasma edge, we impose the same boundary conditions on $\delta E_{m}(r)$ as apply to $E_{m}^{(0)}(r)$. Substituting Eq. (43) into Eq. (41) and expanding to first order in $\delta E_{m}$ and $\delta \omega$, we obtain

$$
G\left(\omega_{0}, E_{m}^{(0)}+\delta E_{m}\right)+\delta \omega \frac{\partial G\left(\omega_{0}, E_{m}^{(0)}\right)}{\partial \omega_{0}}
$$




$$
=-\sum_{j}\left[\delta E_{m}\left(r_{s, j}^{+}\right)-\delta E_{m}\left(r_{s, j}^{-}\right)\right] C_{m}^{(0)}\left(r_{s, j}\right)+i \int_{0}^{a} d r E_{m}^{(0)} L_{k}\left(\omega_{0}\right) E_{m}^{(0)}
$$

where we evaluate

$$
\begin{aligned}
G\left(\omega_{0}, E_{m}^{(0)}+\delta E_{m}\right)= & 2 \mathcal{P} \int_{0}^{a} d r r\left\{\left[r^{2}\left(\frac{d E_{m}^{(0)}}{d r}\right)\left(\frac{d \delta E_{m}}{d r}\right)+\left(m^{2}-1\right) E_{m} \delta E_{m}\right]\left(\frac{\omega_{0}^{2}}{v_{A}^{2}}-k_{\| m}^{2}\right)\right. \\
& \left.-\omega_{0}^{2} r E_{m}^{(0)} \delta E_{m} \frac{d}{d r}\left(\frac{1}{v_{A}^{2}}\right)\right\}
\end{aligned}
$$

Integrating by parts in Eq. (45) and recognizing that $E_{m}^{(0)}$ satisfies Eq. (36) with its right-hand side set equal to zero, we see that

$$
G\left(\omega_{0}, E_{m}^{(0)}+\delta E_{m}\right)=-2 \sum_{j}\left[\delta E_{m}\left(r_{s, j}^{+}\right)-\delta E_{m}\left(r_{s, j}^{-}\right)\right] C_{m}^{(0)}\left(r_{s, j}\right)
$$

Therefore. we can rewrite Eq. (44) as

$$
\delta \omega \frac{\partial G\left(\omega_{0}, E_{m}^{(0)}\right)}{\partial \omega_{0}}=\sum_{j}\left[\delta E_{m}\left(r_{s, j}^{+}\right)-\delta E_{m}\left(r_{s, j}^{-}\right)\right] C_{m}^{(0)}\left(r_{s, j}\right)+i \int_{r_{1}}^{r_{2}} d r E_{m}^{(0)} L_{k}\left(\omega_{0}\right) E_{m}^{(0)}
$$

Comparing the terms on the right-hand sides of Eqs. (44) and (4T), we see that the term containing the discontinuity in $\delta E_{m}$ has changed signs relative to the $L_{k}$ term.

In order to obtain the discontinuity in $\delta E_{m}$ at the singular points $r_{s, j}$, we note that in the vicinity of such a point. Eq. (36) can be written as

$$
\frac{d}{d r}\left[r^{3}\left(\frac{\omega^{2}}{v_{A}^{2}}-k_{\| m}^{2}\right) \frac{d E_{m}}{d r}\right] \equiv 0
$$

from which we obtain

$$
\frac{d E_{m}(r)}{d r} \cong \frac{C_{m}^{(0)}\left(r_{s}\right)}{r^{3}\left[\omega^{2} / v_{A}^{2}(r)-k_{\| m}^{2}(r)\right]}
$$

For $\omega=\omega_{0}$, the result in Eq. (49) matches the lowest-order solution $E_{m}^{(0)}$. We now apply the causality condition that the frequency is formally in the upper half-plane; i.e., $\omega=\omega_{0}+i \eta$, with $0<\eta \ll\left|\omega_{0}\right|$. This allows us to integrate Eq. (49) around the singular point $r_{s}$. 
Treating the discontinuity in the wave function as a first-order perturbation then yields

$$
\begin{aligned}
\delta E_{m}\left(r_{s, j}^{+}\right)-\delta E_{m}\left(r_{s, j}^{-}\right) & =\frac{C_{m}^{(0)}\left(r_{s, j}\right)}{r_{s, j}^{3}} \lim _{\eta \rightarrow 0} \int_{r_{s, j}^{-}}^{r_{s, j}^{+}} d r \frac{1}{\left[\frac{\left(\omega_{0}+i \eta\right)^{2}}{v_{4}^{2}(r)}-k_{\| m}^{2}(r)\right]} \\
& =\frac{-i \pi}{r_{s, j}^{3}} \frac{\operatorname{sgn}\left(\omega_{0}\right) C_{m}^{(0)}\left(r_{s, j}\right)}{\left|\frac{\partial}{\partial r}\left[\omega_{0}^{2} / v_{A}^{2}\left(r_{s, j}\right)-k_{\| m}^{2}\left(r_{s, j}\right)\right]\right|}
\end{aligned}
$$

where $\operatorname{sgn}\left(\omega_{0}\right)$ is the sign of $\omega_{0}$. Substituting Eq. (49) into Eq. (46), we finally obtain

$$
\frac{\delta \omega}{\omega_{0}}\left[\omega_{0} \frac{\partial G\left(\omega_{0}, E_{m}^{(0)}\right)}{\partial \omega_{0}}\right]=-i \pi \sum_{j} \frac{\operatorname{sgn}\left(\omega_{0}\right)\left[C_{m}^{(0)}\left(r_{s, j}\right)\right]^{2}}{r_{s, j}^{3}\left|\frac{\partial}{\partial r}\left(\omega_{0}^{2} / v_{A}^{2}-k_{\| m}^{2}\right)\right|_{r=r_{s, j}}}+i \int_{0}^{a} d r E_{m}^{(0)} L_{k}\left(\omega_{0}\right) E_{m}^{(0)} .
$$

Therefore, as long as the inequality $\omega_{0} \partial G\left(\omega_{0}, E_{m}^{(0)}\right) / \partial \omega_{0}=\partial Q\left(\omega_{0}, \delta \Phi_{0}\right) / \partial \omega_{0}>0$ holds, and treating the resonant mode structure as a perturbation, we find that the cuntinuum resonance gives rises to a damping contribution; i.e.. $\operatorname{Im}(\delta \omega)<0$ from the first term in Eq. (51). This dissipation counteracts possible destabilizing effects from the $L_{k}$ second term. Marginal stability occurs when the right-hand side of Eq. (51) vanishes.

Note that, for a nonsingular perturbation. the inequality $\omega_{0}\left(\partial G / \partial \omega_{0}\right)>0$ is indeed valid [cf. Eq. (20)]. However, with a singular perturbation $\delta E_{m}$, we need to invoke a basic "perturbation assumption," namely, that the contribution to $\partial G / \partial \omega$ coming from near the singular region, as obtained by analytic continuation, is small compared to the bulk contribution. In the kinetic integral of Eq. (51) the "perturbation assumption" also allows us to neglect integration regions near the resonances $r_{s j}$, where a singular response is otherwise possible.

\section{B. Formalism in Toroidal Geometry}

Now we proceed to treat the general coupled-mode problem in toroidal geometry. We consider the system of coupled equations for the TAE mode as given in Eq. (35), with the same boundary conditions as in the preceding discussion, namely, $E_{m}(a)=0$ at the plasma edge and regularity of $E_{m}(r)$ at the magnetic axis as $r \rightarrow 0$. 
As before, ws define the "flux" quantities $C_{m}(r)$, as given by

$$
C_{m}(r)=r^{3} \sum_{n} D_{m n}(r, \omega) \frac{d E_{n}}{d r}
$$

with

$$
D_{m n}(r, \omega)=\left(\frac{\omega^{2}}{v_{A}^{2}}-k_{\| m}^{2}\right) \delta_{m, n}+\hat{\varepsilon}(r)\left(\frac{\omega^{2}}{v_{A}^{2}}\right)\left(\delta_{m+1, n}+\delta_{m-1, n}\right)
$$

Equation (52) can be formally inverted as

$$
\frac{d E_{m}}{d r}=\frac{1}{r^{3}} \sum_{n} \frac{D^{m n}(r, \omega) C_{n}(r)}{\|D(r, \omega)\|}
$$

where $\|D(r, \omega)\|$ represents the determinant of the $m \times n$ matrix whose elements are $D_{m n}$, and $D^{m n}$ is the cofactor of the element $D_{m n}$; i.e.,

$$
\sum_{q} D^{m q} D_{q n}=\delta_{m, n}\|D\|
$$

The second-order differential eigenmode equation (35) can now be rewritten as two first-order equations. one being Eq. (54) and the other being

$$
\frac{d C_{m}(r)}{d r}=\left(m^{2}-1\right)\left(\frac{\omega^{2}}{v_{A}^{2}}-k_{\| m}^{2}\right) r E_{m}-r^{2} E_{m} \frac{d}{d r}\left(\frac{\omega}{v_{A}}\right)^{2}-i L_{k}(\omega) E_{m} .
$$

In terms of this formalism, if the kinetic operator $L_{k}$ is neglected temporarily, we see that the toroidal shear. Alfven continuum corresponds to those points for a given frequency. $\omega$ where the determinant of the coefficients of the highest-order radial derivative terms in the eigenmode equations vanishes. In other words. the eigenmode equation is singular when the coefficients of the terms involving second-order radial derivatives of the wave function components $E_{m}$ lead to a singular matrix equation. This happens when the determinant vanishes: $\|D(r, \omega)\|=0$. For frequencies in the continuum. therefore. the inversion from $C_{m}$ back to $E_{m}$ fails, unless analytic continuation is employed. Thus, for a given frequency $\omega$, continuum resonance interactions occur at $r=r_{s, j}$ where the condition $\left\|D\left(\omega, r_{s, j}\right)\right\|=0$ holds. If Eq. (54) is integrated in the vicinity of such a singular point $r_{s, j}$, it is clear that 
the wave function $E_{m}(r)$ is logarithmically divergent at $r=r_{s, j}$ if $\left(\partial / \partial r_{s, j}\right)\left\|D\left(\omega, r_{s, j}\right)\right\| \neq 0$. However, from Eq. (56), we see that the flux $C_{m}(r)$ is continuous at the singular points.

To derive an expression for the continuum damping rate, we follow the same procedure as has been described for the cylindrical case, but appropriately generalized to take into account the toroidal coupling of modes. Multiply Eq. (35) by $E_{m}(r)$, sum over mode numbers $m$, and integrate from $r=0$ out to $r=a$ with the exclusion of the small intervals $\left(r_{s, j}-\delta, r_{s, j}+\delta\right)$ centered at the singular points $r_{s, j}$ to obtain the exact relationship

$$
G\left(\omega, E_{m}\right)=-\sum_{m, j}\left[E_{m}\left(r_{s, j}^{+}\right)-E_{m}\left(r_{s, j}^{-}\right)\right] C_{m}\left(r_{s, j}\right)+i \sum_{m} \int_{0}^{a} d r E_{m} L_{k}(\omega) E_{m}
$$

with the definition of the quadratic form as

$$
\begin{gathered}
G\left(\omega \cdot E_{m}\right) \equiv \sum_{m} \mathcal{P} \int_{0}^{a} d r r\left\{\left[r^{2}\left(\frac{d E_{m}}{d r}\right)^{2}+\left(m^{2}-1\right) E_{m}^{2}\right]\left(\frac{\omega^{2}}{v_{A}^{2}}-k_{\| m}^{2}\right)-r E_{m}^{2} \frac{d}{d r}\left(\frac{\omega^{2}}{v_{A}^{2}}\right)\right. \\
\left.+r^{2} \hat{\xi}(r)\left(\frac{\omega^{2}}{v_{A}^{2}}\right) \frac{d E_{m}}{d r}\left(\frac{d E_{m+1}}{d r}+\frac{d E_{m-1}}{d r}\right)\right\} .
\end{gathered}
$$

Equations (5T) and (58) are the obvious toroidal generalizations of Eqs. (41) and (42), with $\mathcal{P}$ designating principal value with respect to the singular points, as before.

Note, incidentally, that Eq. (58) can be rewritten as

$$
\begin{aligned}
\frac{1}{\omega} G\left(\omega, E_{m}\right) & =\omega \mathcal{P} \int_{0}^{a} d r r \int_{0}^{2 \pi} \frac{d \theta}{2 \pi}\left[\sum_{m} \frac{d E_{m}(r)}{d r} e^{-i m \theta}\right]^{2}[1+2 \hat{\Xi}(r) \cos \theta]\left(\frac{r}{v_{A}}\right)^{2} \\
& -\sum_{m} \mathcal{P} \int_{0}^{a} d r r\left\{\frac{r^{2} k_{\| m}^{2}}{\omega}\left(\frac{d E_{m}}{d r}\right)^{2}\right. \\
& \left.-\left(\frac{\omega}{v_{A}^{2}}-\frac{k_{\| m}^{2}}{\omega}\right)\left(m^{2}-1\right) E_{m}^{2}+\omega r E_{m}^{2} \frac{d}{d r}\left(\frac{1}{v_{A}^{2}}\right)\right\}
\end{aligned}
$$

with $E_{m}$ real and $E_{-m}=E_{m}$. For $\hat{\varepsilon}(r)<1 / 2$, Eq. (59) shows explicitly that for our eigenmode equation, the inequality

$$
\frac{\partial}{\partial \omega}\left[\frac{G\left(\omega, E_{m}\right)}{\omega}\right]>0
$$


holds for an arbitrary non-singular function $E_{m}$ if the density has a radially non-increasing profile, $\frac{d}{d r}\left(\frac{1}{v_{A}^{2}}\right) \leqq 0$. (Strictly speaking, since $E_{m}$ is singular at ihe resonance points, the positive definiteness in Eq. (60) requires that our perturbation assumption, mentioned earlier, also be satisfied.) Then, if $G\left(\omega, E_{m}\right)=0$, from Eq. (60) we have $\omega^{-1} \partial G\left(\omega, E_{m}\right) / \partial \omega>0$, which is equivalent to positive wave energy.

Next, introduce the perturbation expansion of Eq. (43). The zeroth-order problem, with $L_{k}$ assumed to be negligible and with $E_{m}^{(0)}(r)$, as well _.. ${ }^{\eta)}(r)$, taken to be continuous at the singular points $r_{s, j}$, yields a real eigenfunction $E_{m}^{(0)}$ and a real eigenfrequency $\omega_{0}$ that satisfy $G\left(\omega_{0}, E_{m}^{(0)}\right)=0$. Then, expand Eq. (57) to first order and use the same type of manipulations as were used in obtaining Eq. (45) to find the toroidal generalization of Eq. (47):

$$
\delta \omega^{\prime} \frac{\partial G\left(\omega_{0}, E_{m}^{(0)}\right)}{\partial \omega_{0}}=\sum_{m, j}\left[\delta E_{m}\left(r_{s, j}^{+}\right)-\delta E_{m}\left(r_{s, j}^{-}\right)\right] C_{m}^{(0)}\left(r_{s, j}\right)+i \sum_{m} \int_{0}^{a} d r E_{m}^{(0)} L_{k}\left(\omega_{0}\right) E_{m}^{(0)}
$$

Again, we obtain the discontinuity in $\delta E_{m}$ by invoking causality to integrate Eq. (54) around a Landau-type contour at the singular points:

$$
\delta E_{m}\left(r_{s, j}^{+}\right)-\delta E_{m}\left(r_{s, j}^{-}\right)=\frac{-i \pi}{r_{s, j}^{3}} \sum_{n} \frac{\operatorname{sgn}\left(\frac{\partial\|D\|}{\partial \omega_{0}}\right) D^{m n}\left(\omega_{0}, r_{s, j}\right) C_{n}^{(0)}\left(r_{s, j}\right)}{\left|\frac{\partial}{\partial r_{s, j}}\left\|D\left(\omega_{0}, r_{s, j}\right)\right\|\right|} .
$$

Finally. substituting into Eq. (61) yields the complex frequency shift $\delta \omega$ as follows:

$$
\begin{aligned}
\delta \omega \frac{\partial G\left(\omega_{0}, E_{m}^{(0)}\right)}{\partial \omega_{0}}=-2 \pi \sum_{j} & {\left[\frac{\operatorname{sgn}\left(\frac{\partial\left\|D\left(\omega_{0}, r_{s, j}\right)\right\|}{\partial \omega_{0}}\right) \sum_{m, n} D^{m n}\left(\omega_{0}, r_{s, j}\right) C_{n}^{(0)}\left(r_{s, j}\right) C_{m}^{(0)}\left(r_{s, j}\right)}{r_{s, j}^{3}\left|\frac{\partial}{\partial r_{s, j}}\left\|D\left(\omega_{0}, r_{s, j}\right)\right\|\right|}\right] } \\
& +i \sum_{m} \int_{0}^{a} d r E_{m}^{(0)} L_{k}\left(\omega_{0}\right) E_{m}^{(0)} .
\end{aligned}
$$

In Appendix B, it is proved in general that the first term on the right-hand side of Eq. (63) is imaginary and negative as long as our perturbation assumption is satisfied. Therefore. since the wave energy is positive, we see that the continuum resonances at $r=r_{s, j}$ lead to damping of the wave. At the end of Appendix $\mathrm{C}$, we indicate that even when the perturbative assumption is not satisfied, no unstable roots can exist. 


\section{Analysis of the Cases of Two-Mode and Three-Mode Coupling}

In practice, only the coupling of a finite number of $m$-modes needs to be considered. For example, when $n=1$ and $q(a) \approx 3$, there is coupling between the $m=1$ and $m=2$ modes in the region where $q=1.5$, and coupling between the $m=2$ and $m=3$ modes in the region where $q=2.5$, so that the analysis can be limited to only three $m$-modes. In this section we will examine how the general formalism of Sec. IV reduces specifically for the simple cases of two-mode coupling and three-mode coupling.

When the number of coupled modes is truncated, it is possible to be quite explicit in the form of the cofactor matrix elements $D^{m n}$ and the determinant $\|D\|$. For the case when only two modes. say. $m$ and $m+1$, are coupled, we find the following expressions:

$$
\begin{gathered}
\|D(\omega, r)\|=\left(\frac{\omega^{2}}{v_{A}^{2}}-k_{\| m}^{2}\right)\left(\frac{\omega^{2}}{v_{A}^{2}}-k_{\| m+1}^{2}\right)-\hat{\xi}^{2}\left(\frac{\omega^{4}}{v_{A}^{4}}\right) \\
D^{m, m}=\left(\frac{\omega^{2}}{v_{A}^{2}}-k_{\| m+1}^{2}\right), D^{m+1, m+1}=\left(\frac{\omega^{2}}{v_{A}^{2}}-k_{\| m}^{2}\right) \\
D^{m, m+1}=D^{m+1, m}=-\hat{\varepsilon}\left(\frac{\omega^{2}}{v_{A}^{2}}\right)
\end{gathered}
$$

where the other elements $D^{m n}$ are zero. Similarly, for the case when only three modes, say, $m-1, m$, and $m+1$, are coupled, we find the following expressions:

$$
\begin{gathered}
\|D(\omega, r)\|=\left(\frac{\omega^{2}}{v_{A}^{2}}-k_{\| m-1}^{2}\right)\left(\frac{\omega^{2}}{v_{A}^{2}}-k_{\| m}^{2}\right)\left(\frac{\omega^{2}}{v_{A}^{2}}-k_{\| m+1}^{2}\right) \\
-\hat{\varepsilon}^{2}\left(\frac{\omega^{4}}{v_{A}^{4}}\right)\left(\frac{\omega^{2}}{v_{A}^{2}}-k_{\| m+1}^{2}\right)-\hat{\varepsilon}^{2}\left(\frac{\omega^{4}}{v_{A}^{4}}\right)\left(\frac{\omega^{2}}{v_{A}^{2}}-k_{\| m-1}^{2}\right) \\
D^{m, m}=\left(\frac{\omega^{2}}{v_{A}^{2}}-k_{i \mid m-1}^{2}\right)\left(\frac{\omega^{2}}{v_{A}^{2}}-k_{\| m+1}^{2}\right) \\
D^{m \pm 1, m \pm 1}=\left(\frac{\omega^{2}}{v_{A}^{2}}-k_{\| m}^{2}\right)\left(\frac{\omega^{2}}{v_{A}^{2}}-k_{\| m \mp 1}^{2}\right)-\hat{\varepsilon}^{2}\left(\frac{\omega^{4}}{v_{A}^{2}}\right) \\
D^{m, m \pm 1}=D^{m \pm 1, m}=-\left(\frac{\omega^{2}}{v_{A}^{2}}-k_{\| m \mp 1}^{2}\right) \frac{\omega^{2}}{v_{A}^{2}} \hat{\varepsilon} .
\end{gathered}
$$


Up to terms of $\mathcal{O}\left(\hat{\varepsilon}^{2}\right)$, one can ascertain that the cofactor elements normalized to the determinant, $D^{m n} /\|D\|$, agree for the two-mode and three-mode coupling cases.

Let us now consider a specific example of a two-mode interaction, namely, the case for the $n=1 \mathrm{TAE}$ mode with the $m=1$ and $m=2$ modes coupling, and with the density taken to be almost constant until near the edge of the plasma, where it decreases rapidly. This example is nearly tractable analytically. Based on our earlier discussion, we expect to have a TAE gap mode with the normalized frequency $\Omega=\left[2 q\left(r_{g}\right)\right]^{-1}$, where we define $\Omega=\omega R_{0} / v_{A}(0)$, whose amplitude has a rapid transition at the gap location $r=r_{g}$. determined by $q\left(r_{g}\right)=1.5$. Here we assume that the value of the plasma density at the gap location is nearly unchanged from its value at the axis. $N_{i}\left(r_{g}\right) \cong N_{i}(0)$, which we will designate as $N_{0}$. Near the edge of the plasma where the density falls rapidly, the $m=2$ mode will resonate with the shear Alfvén continuum at the singular point $r=r_{s}$, where $\Omega=\left[N_{0} / N\left(r_{s}\right)\right]^{1 / 2}\left[1-2 / q\left(r_{s}\right)\right]$.

We will first neglect the resonance and develop an analytic description for the zerothorder MHD gap mode. To the extent that $v_{A}^{2}$ is indeed constant, it follows from Eq. (56) that $d C_{1} / d r=0$, and since $C_{1}=0$ is required at the origin, we may take $C_{1}(r)=0$ everywhere. Then. from Eq. (54) and Eq. (64), we have

$$
\begin{aligned}
& \frac{d E_{1}}{d r}=-\frac{\widehat{\varepsilon} \Omega^{2} R_{0}^{2} C_{2}(r)}{r^{3}\left[\left(\Omega^{2}-\kappa_{1}^{2}\right)\left(\Omega^{2}-\kappa_{2}^{2}\right)-\widetilde{\varepsilon}^{2} \Omega^{4}\right]} \\
& \frac{d E_{2}}{d r}=\frac{\left(\Omega^{2}-\kappa_{1}^{2}\right) R_{0}^{2} C_{2}(r)}{r^{3}\left[\left(\Omega^{2}-\kappa_{1}^{2}\right)\left(\Omega^{2}-\kappa_{2}^{2}\right)-\widehat{\varepsilon}^{2} \Omega^{4}\right]}
\end{aligned}
$$

where $\kappa_{m}^{2}=[1-m / q(r)]^{2}$. For a constant density profile, the structure of the dispersion relation in the limit of small $\hat{\varepsilon}$ can be extracted from Eq. (66b). Integrating Eq. (66b) from the origin to $r=a$ and applying the boundary conditions $E_{2}(a)=0=E_{2}(0)$, we obtain

$$
E_{2}(a)-E_{2}(0)=0=\int_{0}^{a} d r \frac{\left(\Omega^{2}-\kappa_{1}^{2}\right) R_{0}^{2} C_{2}(r)}{r^{3}\left[\left(\Omega^{2}-\kappa_{1}^{2}\right)\left(\Omega^{2}-\kappa_{2}^{2}\right)-\widetilde{\varepsilon}^{2} \Omega^{4}\right]}=I_{1}+I_{2}
$$

where we have rewritten the integral in terms of two other integrals,

$$
I_{1}=\int_{0}^{a} d r \frac{R_{0}^{2} C_{2}(r)}{r^{3}}\left[\frac{\left(\Omega^{2}-\kappa_{1}^{2}\right)}{\left[\left(\Omega^{2}-\kappa_{1}^{2}\right)\left(\Omega^{2}-\kappa_{2}^{2}\right)-\hat{\varepsilon}^{2} \Omega^{4}\right]}-\frac{\left(\Omega_{0}^{2}-\kappa_{1}^{2}\right)}{\left[\left(\Omega_{0}^{2}-\kappa_{1}^{2}\right)\left(\Omega_{0}^{2}-\kappa_{2}^{2}\right)-\tilde{\varepsilon}^{2} \Omega_{0}^{4}\right]}\right]
$$




$$
I_{2}=\int_{0}^{a} d r \frac{R_{0}^{2} C_{2}(r)}{r^{3}}\left[\frac{\left(\Omega_{0}^{2}-\kappa_{1}^{2}\right)}{\left[\left(\Omega_{0}^{2}-\kappa_{1}^{2}\right)\left(\Omega_{0}^{2}-\kappa_{2}^{2}\right)-\tilde{\varepsilon}^{2} \Omega_{0}^{4}\right]}\right]
$$

with $\Omega_{0}=1 / 3$ being the normalized frequency at which the $m=1$ and 2 modes intersect in the cylindrical limit. This separation into two integrals is convenient for the purpose of isolating the behavior near the continuum resonance.

The principal contribution to the $I_{1}$ integral comes from the gap region near $q=3 / 2$, where the coupled mode behavior is still well described if we take $C_{2}(r), \widehat{\varepsilon}(r)$, and $r^{3}$ to be constant, evaluated at $r=r_{g}$. Away from the gap location, the integrand of $I_{1}$ tends to become small. Hence we can approximately evaluate the $I_{1}$ integral by expanding its integrand about the gap location: let $\Omega=\Omega_{0}+\delta \Omega$, with $\Omega_{0}=1 / 3$, and let $q(r)=1.5+\delta q$. Also, expand the parallel wave numbers as

$$
\kappa_{1}^{2} \cong\left(1-\frac{1}{\frac{3}{2}+\delta q}\right)^{2} \cong \frac{1}{9}+\frac{8}{2 \tau} \delta q \cdot \kappa_{2}^{2} \cong\left(1-\frac{2}{\frac{3}{2}+\delta q}\right)^{2} \cong \frac{1}{9}-\frac{16}{27} \delta q .
$$

Then we have

$$
\begin{aligned}
I_{1} & \cong \frac{2 \tau R_{0}^{2} C_{2}\left(r_{g}\right)}{8 r_{g}^{3} q^{\prime}\left(r_{g}\right)} \int_{-\infty}^{\infty} d x\left[\frac{(x-y)}{\left[(2 x+y)(x-y)+\left(\hat{\varepsilon}\left(r_{g}\right) \Omega_{0}^{2}\right)^{2}\right]}-\frac{x}{\left[2 x^{2}+\left(\hat{\varepsilon}\left(r_{g}\right) \Omega_{0}^{2}\right)^{2}\right]}\right] \\
& =-\frac{81 \sqrt{2} \pi \delta \Omega R_{0}^{2} C_{2}\left(r_{g}\right)}{32 \Omega_{0} \hat{\varepsilon}\left(r_{g}\right) r_{g}^{3} q^{\prime}\left(r_{g}\right)\left[1-\frac{9}{2}\left(\frac{\delta \Omega}{\Omega_{0} \hat{\varepsilon}\left(r_{g}\right)}\right)^{2}\right]^{1 / 2}}
\end{aligned}
$$

where $x=8(\delta q) / 2 \tau$ and $y=2(\delta \Omega) / 9 \Omega_{0}$.

The integral $I_{2}$ has hardly any contribution from the gap region. since in its integrand. the numerator is nearly odd about the point $r=r_{g}$, whereas the denominator is nearly even. The contribution to this integral occurs away from the gap region, i.e., where the coupling factor $\hat{\Xi}$ can be treated as small. Hence we can approximate $I_{2}$ as

$$
I_{2} \cong \mathcal{P} \int_{0}^{a} d r \frac{R_{0}^{2} C_{2}(r)}{r^{3}\left(\Omega_{0}^{2}-\kappa_{2}^{2}\right)}
$$

In order to evaluate Eq. (72) for $I_{2}$, we need to replace $C_{2}(r)$ by a function $\tilde{C}_{2}(r)$ that approximately satisfies the governing differential equation, Eq. (36) with $L_{k} \rightarrow 0$, as well 
as the proper boundary conditions. Away from the gap region where the modes become uncoupled (i.e., in the $\widehat{\varepsilon}=0$ limit), the appropriate function is given by

$$
\widetilde{C}_{2}(r)=\frac{r^{3}}{R_{0}^{2}}\left(\Omega_{0}^{2}-\kappa_{2}^{2}\right) \frac{d \tilde{E}_{2}}{d r} .
$$

The boundary conditions are taken to be $\tilde{E}_{2}(0)=\tilde{E}_{2}(a)=0$ and $\tilde{C}_{2}\left(r_{g}\right)=C_{2}\left(r_{g}\right)$. What makes it possible to have a solution $\tilde{E}_{2}(r)$ that satisfies the eigenmode equation and also satisfies these three boundary conditions is the fact that, since the equation has a singular point at $r=r_{g}$, it admits solutions with a discontinuity (the "continuum" solutions). The solution $\tilde{E}_{2}(r)$ may be constructed as a linear combination, $\tilde{E}_{2}(r)=E_{c}(r)+\alpha E_{d}(r)$, of a continuous solution, $E_{c}(r)$, and a discontinuous solution, $E_{d}(r)$. Even though $E_{c}(r)$ diverges logarithmically at $r=r_{g}$, it is "continuous" in the sense that $E_{c}\left(r_{g}-\delta\right)=E_{c}\left(r_{g}+\delta\right)$, as $\delta / r_{g} \rightarrow 0$. whereas for the discontinuous solution. $E_{d}\left(r_{g}-\delta\right) \neq E_{d}\left(r_{g}+\delta\right)$. Numerically, one obtains the continuous solution by integrating Eq. (36) with $\Omega=\Omega_{0}$ from $r=0$, with the boundary condition $E_{c}(0)=0$, up to $r=r_{g}^{-}$(with $r_{g}^{ \pm}=r_{g} \pm \delta$, for $\delta \ll 1$ ) and then continuing the integration from $r=r_{g}^{+}$with the continuity condition $E_{c}\left(r_{g}^{-}\right)=E_{c}\left(r_{g}^{+}\right)$out to the edge of the plasma at $r=a$. The discontinuous solution, which is set equal to zero for $0 \leqq r \leqq r_{g}$, is obtained by integrating from the point $r=r_{g}^{+}$, with the conditions $E_{d}\left(r_{3}^{+}\right)=1$ and $C_{d}\left(r_{g}^{+}\right)=0$. out to the plasma edge. After we apply the edge boundary condition $\tilde{E}_{2}(a)=0$, we find $\tilde{E}_{2}(r)=E_{c}(r)-\left[E_{c}(a) / E_{d}(a)\right] E_{d}(r)$. The solution $\tilde{E}_{2}(r)$ so obtained, and its corresponding $\widetilde{C}_{2}(r)$, will match, to within $\mathcal{O}(\varepsilon)$, the correct solution in the domains $0 \leq r<r_{g}^{-}$and $r_{g}^{+}<r \leq a$. In the narrow interval in the vicinity of $r_{g}$. the solution $\tilde{E}_{2}(r)$ will differ from the true solution $E_{2}(r)$, but the function $\widetilde{C}_{2}(r)$, which is continuous, will remain close to the exact $C_{2}(r)$, since the condition $\tilde{C}_{2}\left(r_{g}\right)=C_{2}\left(r_{g}\right)$ has been imposed. Finally, using this approximate solution, we reduce Eq. (72) for the integral $I_{2}$ to the following result:

$$
I_{2} \cong \tilde{E}_{2}\left(r_{g}^{-}\right)-\tilde{E}_{2}\left(r_{g}^{+}\right)=\frac{E_{c}(a)}{E_{d}(a)}
$$


The value of the ratio in Eq. (74) is obtained numerically.

Using Eqs. (67), (71), and (74), we are able to obtain the frequency shift

$$
\frac{\delta \Omega}{\Omega_{0}}=\frac{16 \sqrt{2} r_{g}^{3} q^{\prime}\left(r_{g}\right) \hat{\varepsilon}\left(r_{g}\right) \sigma}{81 \pi a^{2}\left[1+\left(\frac{16 r_{g}^{3} q^{\prime}\left(r_{g}\right) \sigma}{27 \pi a^{2}}\right)^{2}\right]^{1 / 2}}
$$

in terms of the dimensionless ratio $\sigma$, where

$$
\sigma \equiv\left[\frac{\tilde{E}_{2}\left(r_{g}^{-}\right)-\tilde{E}_{2}\left(r_{g}^{+}\right)}{\tilde{C}_{2}\left(r_{g}\right)}\right]\left(\frac{a}{R_{0}}\right)^{2} .
$$

A plot of this ratio $\sigma$ as a function of $q(a)$ for safety factor profiles of the form $q(r)=$ $1+[q(a)-1](r / a)^{2}$ is shown in Fig. 4 for a density profile that is constant out to the plasma edge. Since $\sigma<0$, the eigenfrequency is downshifted from the gap frequency, i.e., $\delta \Omega<0$. For these same quadratic $q(r)$ profiles. Eq. (75) reduces to the following simple formula. which exnibits the dependence of $\delta \Omega$ on the inverse aspect ratio $a / R_{0}$, the edge safety factor $q(a)$, and the discontinuity parameter $\sigma$ :

$$
\delta \Omega=\frac{a}{R_{0}} \frac{\frac{20}{\pi 3^{5}} \frac{a}{(q(a)-1]^{3 / 2}}}{\sqrt{1+\left[\frac{8}{27 \pi} \frac{\sigma}{(q(a)-1)}\right]^{2}}} .
$$

In Fig. 5. the numerically obtained values for the normalized frequency shift $\delta \Omega\left(R_{0} / a\right)$ are plotted as a function of the inverse aspect ratio, for several values of $q(a)$. As $a / R_{0} \rightarrow 0$, Fig. 5 shows that the values for $\delta \Omega\left(R_{0} / a\right)$ exactly approach the respective values predicted by the semi-analytical theory of Eq. (77).

Having obtained an analytic description for the zeroth-order MHD gap mode, we next consider the damping caused by the shear Alfven continuum resonance of the $m=2$ harmonic near the plasma edge. For the sake of definiteness, we take a density profile that is flat over most of the plasma and then decreases linearly near the edge:

$$
N_{i}(r)=\left\{\begin{array}{l}
N_{0}, \text { for } r<a(1-\Delta) \\
N_{0}\left(1-\frac{r-a}{\Delta}\right), \text { for } a(1-\Delta)<r<a .
\end{array}\right.
$$


For simplicity, we shall neglect the radial variation of the shear at the resonance point $r=r_{s}$. (Recall that we have assumed $q(a)<3$, and hence the natural resonance of the $m=2$ mode at $q \approx 3$ due to $q(r)$ variation when the density is flat has been eliminated from our example problem.) At the resonance point only the $m=2$ mode will resonate, and so we may neglect $\mathcal{O}(\varepsilon)$ coupling terms at resonance. Then Eq. (63) yields for the continuum damping rate

$$
\frac{\gamma}{\omega_{0}}=-\pi \frac{\Delta\left[v_{A}(0) C_{2}\left(r_{s}\right)\right]^{2}}{\left(r_{s} \omega_{0}\right)^{3} \frac{\partial G}{\partial \omega_{0}}}
$$

where

$$
\omega_{0} \frac{\partial G}{\partial \omega_{0}} \cong 2\left[\frac{\omega_{0}}{v_{A}(0)}\right]^{2} \int_{0}^{a} d r r^{3}\left[\left(\frac{d E_{1}}{d r}\right)^{2}+\left(\frac{d E_{2}}{d r}\right)^{2}\right] .
$$

Since the integrand in Eq. (80) tends to be localized about the gap location, we calculate its contribution by writing $\Omega=\Omega_{0}+\delta \Omega$, with $\Omega_{0}=1 / 3$, and $q(r)=1.5+\delta q$, as before, and then expanding Eqs. (66a) and (66b) for $d E_{1} / d r$ and $d E_{2} / d r$ :

$$
\begin{aligned}
& \frac{d E_{1}}{d r} \cong \frac{\hat{\varepsilon}\left(r_{g}\right) \Omega_{0}^{2} R_{0}^{2} C_{2}\left(r_{g}\right)}{r_{g}^{3}\left[\left(\frac{8 \delta q}{27}-\frac{2 \delta \Omega}{9 \Omega_{0}}\right)\left(\frac{16 \delta q}{27}+\frac{2 \delta \Omega}{9 \Omega_{0}}\right)+\widetilde{\varepsilon}^{2}\left(r_{g}\right) \Omega_{0}^{4}\right]} \\
& \frac{d E_{2}}{d r} \cong \frac{\left(\frac{8 \delta q}{27}-\frac{2 \delta \Omega}{9 \Omega_{0}}\right) R_{0}^{2} C_{2}\left(r_{g}\right)}{r_{g}^{3}\left[\left(\frac{8 \delta q}{27}-\frac{2 \delta \Omega}{9 \Omega_{0}}\right)\left(\frac{16 \delta q}{27}+\frac{2 \delta \Omega}{9 \Omega_{0}}\right)+\widehat{\varepsilon}^{2}\left(r_{g}\right) \Omega_{0}^{4}\right]} .
\end{aligned}
$$

Now change the variable of integration in Eq. (80) from $r$ to $\delta q$ and extend the range of integration to find

$$
\int_{0}^{a} d r r^{3}\left(\frac{d E_{1}}{d r}\right)^{2} \cong 2 \int_{0}^{a} d r r^{3}\left(\frac{d E_{2}}{d r}\right)^{2} \cong \frac{27 \pi\left[R_{0}^{2} C_{2}\left(r_{g}\right)\right]^{2}}{16 \sqrt{2} \Omega_{0}^{2} \hat{\varepsilon}\left(r_{g}\right) r_{g}^{3} q^{\prime}\left(r_{g}\right)\left[1-\frac{9}{2}\left(\frac{\delta \Omega}{\Omega_{0} \widehat{\varepsilon}\left(r_{g}\right)}\right)^{2}\right]^{3 / 2}}
$$

Therefore, since $\Omega_{0}^{2}=1 / 9$, using Eqs. (79) and (75) and the approximation $r_{s} \approx a$, we obtain

$$
\begin{aligned}
\frac{\gamma}{\omega_{0}} & =-\left(\frac{\Delta}{r_{s}}\right)\left(\frac{16 \sqrt{2} r_{g}^{3} q^{\prime}\left(r_{g}\right) \hat{\varepsilon}\left(r_{g}\right)}{81 \Omega_{0}^{2} r_{s}^{2}}\right)\left[\frac{C_{2}\left(r_{s}\right)}{C_{2}\left(r_{g}\right)}\right]^{2}\left[1-\frac{9}{2}\left(\frac{\delta \Omega}{\Omega_{0} \hat{\varepsilon}\left(r_{g}\right)}\right)^{2}\right]^{3 / 2} \\
& =-\frac{\left(\frac{\Delta}{a}\right)\left(\frac{8 \sqrt{2} \hat{\varepsilon}\left(r_{g}\right)}{9\left[q(a)-\Sigma_{:}\right.}\right)\left[\frac{C_{2}\left(r_{s}\right)}{C_{2}\left(r_{g}\right)}\right]^{2}}{\left[1+\left(\frac{8 \sigma}{27 \pi[q(a)-1]}\right)^{2}\right]^{3 / 2}}
\end{aligned}
$$


where $\hat{\varepsilon}\left(r_{g}\right)=\left(5 / 2^{3 / 2}\right)\left(a / R_{0}\right)[q(a)-1]^{-\frac{1}{2}}$ for the quadratic $q(r)$ profile being considered here. Numerically we find that $\left[C_{2}\left(r_{s}\right) / C_{2}\left(r_{g}\right)\right]^{2}$ assumes a value somewhat less than unity; its precise value is shown in Fig. 6 for various aspect ratios.

\section{Numerical Solution}

In this section we discuss the numerical solution of Eq. (35) for the case of three-mode coupling, specifically, for the $n=1 \mathrm{TAE}$ mode when the $m=1,2$, and 3 modes are taken into account. The coupled equations for these modes, Eqs. (54) and (56), with the determinant $\|D(\omega, r)\|$ and matrix elements $D^{m, n}$ given by Eq. (65), were numerically solved with a standard error-predictor integration routine.

In order to integrate the set of six first-order differential equations for $E_{m}(r)$ and $C_{m}(r)$, we need to impose the appropriate boundary conditions. At the edge of the plasma, since the field quantity $E_{m}(r)=\Phi_{m}(r) / r$ is essentially the plasma displacement and we consider fixed boundary perturbations, we have $E_{m}(a)=0$. The examination of boundary conditions near the magnetic axis $r=0$ is somewhat more complicated due to the mode coupling. Without any toroidicity coupling (and neglecting kinetic contributions), the various wave functions $E_{m}$ satisfy the following equation near the axis, where the density and safety factor may be taken to be approximately constant (with the density and safety factor both taken as unity at the origin in this discussion):

$$
\frac{d}{d r}\left(r^{3} \frac{d E_{m}}{d r}\right)-\left(m^{2}-1\right) r E_{m} \cong 0 .
$$

Equation (85) yields

$$
E_{m}(r) \propto r^{-1 \pm|m|}
$$

as the regular solutions near the origin. However, this result is slightly modified when toroidal coupling is included. Specifically, from Eq. (35) we see that $E_{m-1}$ in the toroidicity coupling term, when $E_{m-1}$ has the form given in Eq. (86) and as long as $d E_{m-1} / d r \neq 0$, will drive 
a finite $E_{m}$ that also has the form given in Eq. (86). Therefore, in this case, the regular solutions near the origin are not independent, and we need to include the driven response in the proper boundary conditions. This can be accomplished by modifying Eq. (85) to the following form:

$$
\frac{d}{d \rho}\left(\rho^{3} \frac{d E_{m}}{d \rho}\right)-\left(m^{2}-1\right) \rho E_{m}+\lambda \frac{d}{d \rho}\left(\rho^{3} \frac{d E_{m-1}}{d \rho}\right) \cong 0
$$

with $\rho=r / a \ll 1$ the normalized minor radius and $\lambda_{m}=\left(5 a / 2 R_{0}\right)\left[\Omega^{2} /\left(\Omega^{2}-\kappa_{m}^{2}\right)\right]$ a constant parameter, with $\kappa_{m}^{2}=(m-1)^{2}$. For $E_{m-1}=\rho^{-2+m}$ near the axis (with $m>2$ ), Eq. (8T) yields the driven response

$$
E_{m}(\rho)=-\frac{(m-1)(m+1)}{2 m} \lambda_{m} \rho^{2} \ln \rho .
$$

In the three-mode coupling problem whose numerical solution will be presented in this section. we will apply the result of Eq. (88) for the $m=3$ mode.

With this information about the behavior of the solutions near the magnetic axis, the six coupled equations for the dependent functions $E_{m}$ and $C_{m}$, for $m=1,2$, and 3 , can be integrated from near the axis at $r=0$ out to the plasma edge at $r=a$, where the $E_{m}$ must vanish. To satisfy the boundary condition at the edge requires knowledge of the solution for the eigenfrequency $w$. This can be achieved by separately performing three complete integrations and then combining their respective results. For the first integration, take $E_{1}$ near the axis (i.e., for $\rho \rightarrow 0$ ) to have the regular form given by Eq. (86), namely, $E_{1}(\rho)=1$. while taking $E_{2}(\rho)=E_{3}(\rho)=0$; denote the solutions obtained in this first case as $E_{m}^{(1)}(r)$ and $C_{m}^{(1)}(r)$. For the second integration, take $E_{2}(r)$ to have its regular form near the axis, viz., $E_{2}(\rho)=\rho$, while $E_{1}$ and $E_{3}$ are integrated so as to satisfy $E_{1}(\rho)=0$ and, from Eq. (88), $E_{3}(\rho)=-(2 \lambda / 3) \rho^{2} \ln \rho$ near the axis; denote the solutions obtained in this second case as $E_{m}^{(2)}(r)$ and $C_{m}^{(2)}(r)$. Finally, for the third integration, take $E_{3}$ to have its regular form $E_{3}(\rho)=\rho^{2}$ near the axis, and take $E_{1}(\rho)=E_{2}(\rho)=0$; denote the solutions obtained in this third case as $E_{m}^{(3)}(r)$ and $C_{m}^{(3)}(r)$. Now construct a linear combination of the separate 
solutions,

$$
E_{m}(r)=\sum_{i=1}^{3} e_{i} E_{m}^{(i)}(r) \quad, \quad C_{m}(r)=\sum_{i=1}^{3} e_{i} C_{m}^{(i)}(r)
$$

where the coefficients $e_{i}$ are to be determined by the boundary conditions $E_{m}(a)=0$. If a frequency $\omega$ can be found which have coefficients $e_{i}$ that make the wave functions $E_{m}$ vanish at the plasma edge, then that $\omega$ is the eigenfrequency and the resulting $E_{m}(r)$ are the eigenfunctions. We summarize the manifold of boundary conditions for $E_{m}^{(i)}$ and $C_{m}^{(i)}$ at $\rho=r / a \ll 1$ as follows:

$$
\begin{array}{lll}
E_{1}^{(1)}(\rho)=1 & E_{1}^{(2)}(\rho)=0 & E_{1}^{(3)}(\rho)=0 \\
E_{2}^{(1)}(\rho)=0 & E_{2}^{(2)}(\rho)=\rho & E_{2}^{(3)}(\rho)=0 \\
E_{3}^{(1)}(\rho)=0 & E_{3}^{(2)}(\rho)=-\left(2 \lambda_{3} / 3\right) \rho^{2} \ln \rho & E_{3}^{(3)}(\rho)=\rho \\
C_{1}^{(1)}(\rho)=0 & C_{1}^{(2)}(\rho)=0 & C_{1}^{(3)}(\rho)=0 \\
C_{2}^{(1)}(\rho)=0 & C_{2}^{(2)}(\rho)=\rho^{3}\left(\Omega^{2}-1\right) & C_{2}^{(3)}(\rho)=0 \\
C_{3}^{(1)}(\rho)=0 & C_{3}^{(2)}(\rho)=\left(\lambda_{3} / 3\right)\left(\Omega^{2}-4\right) \rho^{4}(1-4 \ln \rho) & C_{3}^{(3)}(\rho)=2\left(\Omega^{2}-4\right) \rho^{4} .
\end{array}
$$

In writing Eq. (90), we have assumed $q(0)=1$ at the magnetic axis.

In our numerical procedure. we first solve for the reduced ideal MHD (i.e., lowest-order) eigenfrequency $\omega_{0}$ for the TAE mode. We begin with the guess $\omega \approx \omega_{\text {gap }} \equiv\left|-m / q\left(r_{3}\right)+1\right|$ $v_{A}\left(r_{3}\right) / R_{0}$. Then we determine the continuum resonant points $r_{s, j}$ that satisfy the equation $\left\|D\left(\omega, r_{s, j}\right)\right\|=0$. Knowing these points, we integrate Eqs. (54) and (56), with the boundary conditions given by Eq. (90), from the origin to $r=r_{s}(1-\delta)$ with $\delta \ll 1$; i.e.. just before the first singular point is encountered. We jump to the other side of the singular point. demanding that both $E_{m}(r)$ and $C_{m}(r)$ be continuous at $r=r_{s}$, and. beginning at $r=$ $r_{s}(1+\delta)$, integrate farther, either to the next singular point or to the end of the integration domain at $r=a$. When the end of the integration is attained, we examine whether a superposition of the $E_{m}^{(i)}$ solutions will satisfy the condition

$$
\sum_{i=1}^{3} e_{i}(\omega) E_{m}^{(i)}(a)=0
$$


The condition of Eq. (91) is equivalent to requiring that the determinant of the matrix, whose elements are the values $E_{m}^{(i)}(a)$, be equal to zero:

$$
\|E(\omega)\|=\left|\begin{array}{lll}
E_{1}^{(1)}(a ; \omega) & E_{1}^{(2)}(a ; \omega) & E_{1}^{(3)}(a ; \omega) \\
E_{2}^{(1)}(a ; \omega) & E_{2}^{(2)}(a ; \omega) & E_{2}^{(3)}(a ; \omega) \\
E_{3}^{(1)}(a ; \omega) & E_{3}^{(2)}(a ; \omega) & E_{3}^{(3)}(a ; \omega)
\end{array}\right|=0 .
$$

If $\|E(w)\| \neq 0$, we make a new prediction $\omega_{\nu}$ for the eigenfrequency according to the prescription

$$
\omega_{\nu}=\frac{\omega_{\nu-1}\left\|E\left(\omega_{\nu-2}\right)\right\|-\omega_{\nu-2}\left\|E\left(\omega_{\nu-1}\right)\right\|}{\left\|E\left(\omega_{\nu-2}\right)\right\|-\left\|E\left(\omega_{\nu-1}\right)\right\|}
$$

where $\nu$ is the iteration index, with $\omega_{\nu-1}$ and $\omega_{\nu-2}$ being the previous two guesses for the eigenfrequency. When, to an appropriate iolerance, the condition $\|E(\omega)\|=0$ is satisfied. then the eigenfrequency $\omega_{0}$ has been attained, and the coefficients $e_{i}$ may be taken to be

$$
\begin{aligned}
& e_{1}=-E_{1}^{(2)}(a) E_{3}^{(3)}(a)+E_{1}^{(3)}(a) E_{3}^{(2)}(a) \\
& e_{2}=E_{1}^{(1)}(a) E_{3}^{(3)}(a)-E_{1}^{(3)}(a) E_{3}^{(1)}(a) \\
& e_{3}=-E_{1}^{(1)}(a) E_{3}^{(2)}(a)+E_{3}^{(1)}(a) E_{1}^{(2)}(a) .
\end{aligned}
$$

Equations (89) and (94) yield the corresponding TAE eigenfunction.

Next, the continuum damping rate is evaluated, with the use of Eq. (63). With the exception of the quantity $\partial G\left(\omega_{0}, E_{m}^{(0)}\right) / \partial \omega_{0}$, the numerical evaluation of the various terms in Eq. (63) is straightforward. The derivative $\partial\left\|D\left(\omega_{0} . r_{s, j}\right)\right\| / \partial r$ can be obtained by finite difference construction, and $C_{m}\left(r_{s, j}\right)$ can be obtained from the superposition $C_{m}\left(r_{s, J}\right)=$ $\Sigma_{1} e_{i} C_{m}^{(i)}\left(r_{s, j}\right)$ of Eq. (89), where the values $C_{m}^{(i)}\left(r_{s, j}\right)$ have been stored from the numerical integration of the differential equations. Also, neglecting the $m=0$ component for simplicity, from Eq. (58) we obtain

$$
\begin{aligned}
\omega \frac{\partial G\left(\omega \cdot E_{m}\right)}{\partial \omega}= & -2 \sum_{m} \omega^{2} \mathcal{P} \int_{0}^{a} \frac{d r r}{v_{A}^{2}}\left\{r^{2}\left(\frac{d E_{m}}{d r}\right)^{2}+\left(m^{2}-1\right)\left(E_{m}\right)^{2}-v_{A}^{2} r E_{m}^{2} \frac{d}{d r}\left(\frac{1}{v_{A}^{2}}\right)\right. \\
& \left.+r^{3} \hat{\varepsilon}(r) \frac{d E_{m}}{d r}\left(\frac{d E_{m+1}}{d r}+\frac{d E_{m-1}}{d r}\right)\right\} .
\end{aligned}
$$


In the limit of small $a / R_{0}$, this expression for $\omega_{0} \partial G\left(\omega_{0}, E_{m}^{(0)}\right) / \partial \omega_{0}$ in Eq. (95) is apparently positive definite for a decreasing density profile. However, near the singular points $r_{s, j}$, we have $\left(d E_{m} / d r\right)^{2} \rightarrow\left(r-r_{s, j}\right)^{-2}$, which lears to a divergent integral in Eq. (95). To obtain a finite value for the integral, a more careful construction of the frequency derivative of the principal value function is needed. The method for such a construction is discussed in Appendix C, which gives an explicit analysis for the case without toroidal coupling and indicates how the procedures developed there can be generalized to the case with toroidal coupling. We obtain a form for $\omega_{0} \partial G\left(\omega_{0}, E_{m}^{(0)}\right) / \partial \omega_{0}$ that is nondivergent, but which in a strict sense has lost its positive definiteness property [see Eq. (C8)]. However, the positive definiteness is still applicable as long as our basic perturbation assumption in fulfilled. Finally, we note that for the three-mode coupling problem, the most useful form for calculating $\partial G\left(\omega_{0} . E_{m}^{(0)}\right) / \partial \omega_{0}$ is given in Eq. (C11) of Appendix C, namely,

$$
\frac{\partial G\left(\omega_{0}, E_{m}^{(0)}\right)}{\partial \omega}=-\frac{\partial\left\|E\left(\omega_{0}\right)\right\|}{\partial \omega} C_{2}^{(0)}(a)
$$

where $\|E\|$ is the determinant defined in Eq. (92).

In Fig. $T$ we show the continuum damping rate. $\gamma_{\text {damp }}$, normalized to the TAE mode frequency $\omega_{0}$, as a function of $q(a)$. the edge safety factor value. for $a / R_{0}=0.25$. This survey was performed for a quadratic $q$-profile of the form

$$
q(r)=q(0)+[q(a)-q(0)]\left(\frac{r}{a}\right)^{2}
$$

with $q(0)=1$ and for three different density profiles: Fig. $7(a)$ is for the profile given by Eq. (78); Fig. 7 (b) is for density profile $N_{i}(r) /\left[1+(r / a)^{2}\right]$, where $N_{i}(r)$ is given by Eq. (78); and Fig. $T(c)$ is for the "smooth" profile

$$
N_{i}(r)=\frac{N_{0}\left[1+\exp \left(\frac{-1+2 \Delta_{1}}{2 \Delta_{2}}\right)\right]}{\left[1+\exp \left(\frac{\left(\frac{r}{a}\right)^{2}-1+2 \Delta_{1}}{2 \Delta_{2}}\right)\right]}
$$

with $\Delta_{1}=0.2$ and $\Delta_{2}=0.05$. The density profile of Eq. (98), normalized to unity at $r=0$, is shown in Fig. (8). When $q(a) \equiv 2.0$, which was the situation examined in Refs. 1-6, the 
$n=1$ TAE mode couples the $m=1$ and 2 poloidal harmonics and is peaked near the surface where $q(r)=1.5$. The damping is then due to the interaction of the $m=2$ component with the continuum near the edge of the plasma. When $q(a)$ is around 3.0 , there is another toroidal coupling, primarily between the $m=2$ and 3 harmonics and near $q(r)=2.5$, whose frequency "gap" is close to the original TAE frequency.

This results in considerable structure in the frequency and damping diagrams in curves of Fig. 7 . The $m=2,3$ structure at the edge can excite the $m=3$ mode, which then either produces large damping due to its excitation and resonance of the $m=2,3$ components, or very little damping, if the eigenfrequency of the components manages to "thread" the gaps. A more detailed study of the threading is exhibited in Figs. 9 and 10. In Fig. 9 the resonance diagram is shown for the profile of Eq. (98), with the parameters of Fig. 7 (c) and with $q(a)=3.165$. Two eigenfrequencies are found, $\Omega=0.389$ and $\Omega=0.313$, as indicated by the horizontal lines in Fig. 9. The upper frequency curve avoids all resonances and has zero damping. We see in Fig. 10(a) that a large $m=3$ component has been excited. The eigenstructure for the lower frequency mode has a negligible $m=3$ component, but the $m=2$ resonance at the edge gives considerable damping with $\gamma / \Omega=-0.14$. In this case the large damping is due to the intersection of the eigenfrequency at a resonance that is very close to the location of the outer gap at $r / a=0.841$. Our perturbation method is suspect here because the eigenfunction can have several singular points and our assumption that the eigenfunction is real can break down. An alternate method to calculate the damping rate in this case is discussed in Appendix D; in general, the modified calculation predicts an even larger damping rate (cf. Fig. 13).

For larger $q(a)$, there is a tendency for the magnitude of the damping rate, $|\gamma / \omega|$, to decrease. The damping rate is particularly small when the density profile is gradually decreasing, as seen in Fig. 7(b).

In all, we see that the magnitude of the damping is sensitive to detailed parameters. 
For the rather flat density profiles of Figs. $7(\mathrm{a})$ and $7(\mathrm{c})$, we typically find $|\gamma / \omega| \gtrsim 0.01$, although rather narrow parameter ranges can be found that avoid resonance and give either particularly low damping or particularly heavy damping. With a density profile that slowly decreases radially, somewhat lower damping rates are found with $|\gamma / \omega|<0.01$.

\section{Discussion}

We now wish to assess the importance of the continuum mode damping calculated in this paper. A description of how to calculate the alpha particle instability drive is contained in Appendix A, with the final formula for estimating the alpha-induced growth rate given in Eq. (A33).

In applying the formula of Eq. (A33) we note that the only significant parameter whose value is flexible in the various foreseen ignition experiments is $\rho_{\alpha} / \ell_{\alpha}$, the ratio of the alpha particle Larmor radius to the alpha particle scale length. The background plasma beta is usually chosen to be as high as possible, commensurate with global MHD stability. The electron temperature $T_{e}$ is usually chosen to be as low as possible while still satisfying the achievement of ignition conditions; for D-T ignition. the temperature is taken to be $10 \mathrm{keV}$. In Table I we list nominal parameters for the TFTR. CIT, and ITER experiments and observe that the central values of the background plasma beta are nearly the same in the various experiments. The ratio $x_{A} \equiv v_{A} / v_{\alpha 0} \propto T_{i}^{1 / 2} / v_{\alpha 0} \beta_{i}^{1 / 2}$ likewise tends to be fixed. for fixed beta and $T$. From Eq. (A26) we note that the alpha particle beta value $\beta_{\alpha}$ is specified as a given function of the temperature, the plasma beta. and the isotopic mix of deuterium and tritium (henceforth it is here assumed that $N_{D}=N_{T}=N_{e} / 2$ ). since the product $N_{e} \tau_{s}$ is only a function of the electron temperature and $\langle\sigma v\rangle$ is only a function of the ion temperature. For the sake of definiteness, we will take a central plasma beta value of $\beta=0.03$, along with $x_{A}=v_{A} / v_{\alpha 0}=0.55$ and $R_{0} / a=3$. The parameter that can vary from machine to machine, 
and from experiment to experiment in a given machine, is

$$
\frac{\tilde{\omega}_{\star_{\alpha}}}{\omega} \simeq \frac{3 R_{0} v_{\alpha 0} \rho_{\alpha 0}}{2 r_{g} v_{A} \ell_{\alpha}}
$$

with $\rho_{\alpha 0}=v_{\alpha 0} / \omega_{c \alpha}$ and $\ell_{\alpha}^{-1}=\frac{\partial}{\partial r}\left|\ln \tilde{\beta}_{\alpha}\right|[($ see Eq. (A26)]. For the purposes of our modelling, we will use the $q$-profile used earlier in this paper, $q(r)=1+[q(a)-1] r^{2} / a^{2}$, so that $r_{g} / a=\left[\frac{1}{2(q(a)-1)}\right]^{1 / 2}$, and we take $q(a) \approx 3$, so that $r_{g} / a=1 / 2$. Then the quantity $\frac{\tilde{\omega}_{*_{\alpha}}}{\omega}$ for a $10 \mathrm{keV}$ plasma can be written as

$$
\frac{\tilde{\omega} *_{\alpha}}{\omega} \approx \frac{16 \rho_{\alpha}}{a} \frac{a}{\ell_{\alpha}}= \begin{cases}1.5 a / \ell_{\alpha} & \text { (TFTR) } \\ 0.8 a / \ell_{\alpha} & \text { (CIT) } \\ 0.6 a / \ell_{\alpha} & \text { (ITER) }\end{cases}
$$

The value of the parameter $a / \ell_{\alpha}$ depends on the temperature profile that is actually achieved in the experiment.

In Fig. 11 we plot the ratio of the growth rate to the oscillation frequency, $\gamma_{\alpha} / \omega$, predicted from Eq. (A33) for a plasma at various temperatures. First, focus on the $\mathrm{T}=10 \mathrm{keV}$ curve in Fig. 11. Note that a $1 \%$ value for $\gamma_{\alpha} / \omega$ requires a relatively steep alpha particle scale length compared to the minor radius $a$ : namely, $\ell_{\alpha} / a \simeq 0.24,0.1 \dot{u}$, and 0.095 for TFTR, CIT and ITER, respectively. A one-percent growth rate can usually be counter-balanced by the Alfvén resonance damping which, as we have observed in Sec. VI, is typically of this magnitude (see Fig. 7). If instability of the $n=1$ mode does arise. the $\ell_{\alpha}$ scale length and also the value of $\beta_{\alpha}$ should change due to instability, with $\beta_{\alpha} \ell_{\alpha}^{2}$ remaining roughly constant. This relaxation should stabilize the mode, and since $\ell_{\alpha} / a$ is small, the alpha particles should still be contained. Perhaps the only difficulty in such a scenario is that the alpha particle heating would become more diffuse, which might influence various ignition scenarios.

After ignition, the plasma temperature should rise. Two extreme possibilities are that either the beta will remain constant or the density will remain constant. The former situation may be plausible when the plasma is near its MHD beta limits. The values of $\gamma_{\alpha} / \omega$ for various values of $T$ are shown in Fig. 11. The solid curves correspond to constant plasma 
density with $T=10,15$, and $20 \mathrm{keV}$. The dashed and dotted curves correspond to the case of a fixed plasma beta of $3 \%$, with $T=15$ and $20 \mathrm{keV}$, and the case of fixed density, respectively. Substantially increased growth rates relative to the real frequency occur at higher temperature in both the constant-density and constant-beta cases.

The most important conclusion to be drawn from the work described in the present paper is that the interaction of the low-mode-number toroidal Alfven eigenmode with the continuum, which is especially like to occur near the plasma periphery due to density variation, leads to dissipation and wave damping. Such continuum dissipation can significantly reduce the growth rate of the alpha particle-destabilized TAE modes. A weakened TAE instability would imply lower wave saturation amplitude levels, which in turn would imply better confinement of the hot alpha particles that fuel the thermonuclear plasma ignition process.

To date. the only estimate of the nonlinear saturation level of the TAE wave amplitude is the analytical prediction that was derived by Berk and Breizman ${ }^{11}$ with the use of wave trapping arguments. Their theory indicates that the saturation amplitude of the perturbed magnetic field, $\delta B$, scales as a fractional power of the ratio of the linear growth $\gamma_{L}$ to the dissipative damping rate $\gamma_{d}$ : viz., for typical parameters, $\delta B / B \approx 1 \times 10^{-5}\left(\gamma_{L} / \gamma_{d}\right)^{2 / 3}$. Clearly, it may be expected that the significant dissipation produced by the continuum resonance interaction would tend to reduce the saturation amplitude and, consequently, the threatening nature of the TAE instability. More detailed comparison with theory and also numerical simulations of the TAE nonlinear behavior are called for. Already, computational studies $^{10}$ of alpha particle orbit confinement in the presence of a TAE wave, albeit nonselfconsistent, are exploring how the alpha particle confinement time varies with the specified amplitude of the wave.

We emphasize that in the present paper we have carefully studied only the $n=1 \mathrm{TAE}$ instability. Higher $n$-numbers may cause more severe instability, particularly if the gap structure is such as to avoid any Alfvén continuum resonance damping, as indicated in 
Ref. 22.

Further investigations are needed with realistic MHD codes for proper calculation of the continuum resonance and the frequency gap structure, which we have observed to be sensitive to details of the profiles. When only a single resonance point is present, the Alfvén continuum resonance damping contribution can readily be incorporated into these large codes by means of the theory developed in the present paper.

\section{Acknowledgments}

The authors acknowledge helpful discussions with Prof. D.E. Baldwin, Dr. B. Breizman, Dr. S.C. Guo, Dr. C.Z. Cheng, Dr. L. Chen, Dr. M. Chu, and Prof. M.N. Rosenbluth. In particular, Dr. G.Y. Fu supplied us with his code for the two-mode coupling case and provided valuable comments.

This work was supported by the U.S. Department of Energy under contract DE-FG0.580ET-53088. One of the authors (ZG) was also partially supported by the Texas Advanced Research Program. 


\section{Appendix A: Hot Particle Response}

Here we solve for the alpha particle kinetic response and show how to obtain an analytic estimate of the alpha particle-induced TAE growth rate.

The alpha particle kinetic response enters the eigenmode equation through the pressure perturbation, as may be seen in Eq. (7). For an anisotropic plasma, the equilibrium pressure can be written in the form

$$
P=p_{\|} \hat{b} \hat{b}+p_{\perp}(1-\hat{b} \hat{b})
$$

where $p_{\|}$and $p_{\perp}$ are the components of the pressure tensor $\mathrm{P}$ along and across the direction (specified by the unit vector $\hat{b}$ ) of the equilibrium magnetic field $\mathbf{B}=\widehat{b} B$. The perturbed pressure is then given by

$$
\delta \mathrm{P}=\delta p_{\|} \hat{\mathbf{b}} \hat{\mathbf{b}}+\delta p_{\perp}(1-\hat{\mathbf{b}} \hat{\mathbf{b}})+\delta B_{\|} \frac{\partial}{\partial B} \mathbf{P}+\frac{\left(p_{\|}-p_{\perp}\right)}{B^{2}}\left(\mathbf{B} \delta \mathbf{B}_{\perp}+\delta \mathbf{B}_{\perp} \mathbf{B}\right)
$$

where the perturbed pressure components $\delta p_{\|}$and $\delta p_{\perp}$ are given as kinetic integrals over the perturbed distribution function $\delta f$ :

$$
\delta p_{\|}=M \int d^{3} v v_{\|}^{2} \delta f, \quad \delta p_{\perp}=M \int d^{3} v \mu B \delta f
$$

From Eq. (A2) we can then obtain

$$
\begin{aligned}
{[1+} & \left.\frac{4 \pi}{B^{2}}\left(p_{\perp}-p_{\|}\right)\right] \frac{\hat{\mathbf{b}}}{B} \times \nabla \cdot \delta \mathrm{P}=\frac{1}{B}\left(\delta p_{\|}-\delta p_{\perp}\right)(\hat{\mathbf{b}} \times \kappa)+\frac{1}{B}\left(\hat{\mathbf{b}} \times \nabla \delta p_{\perp}\right) \\
& +\frac{\hat{\mathbf{b}}}{B} \times \nabla \cdot\left(\delta B_{\|} \frac{\partial}{\partial B} \mathrm{P}\right)+(\hat{\mathbf{b}} \times \nabla B) \hat{\mathbf{b}} \cdot \nabla\left(\frac{p_{\|}-p_{\perp}}{B^{2}}\right) \\
& -2 \delta B_{\|}\left(\frac{p_{\|}-p_{\perp}}{B^{2}}\right)(\hat{\mathbf{b}} \times \boldsymbol{\kappa})+\left(\frac{p_{\|}-p_{\perp}}{B^{2}}\right) \frac{\hat{\mathbf{b}}}{B} \times\left[\nabla\left(B \delta B_{\|}\right)-4 \pi i \omega \delta \mathbf{v}_{E}\right] .
\end{aligned}
$$

In the low-beta limit of interest here, we neglect the terms $4 \pi p_{\|, \perp} / B^{2} \ll 1$ and set $\delta B_{\|} \rightarrow 0$ in Eq. (A4), so that we have

$$
\nabla \cdot\left(\frac{\hat{\mathrm{b}}}{B} \times \nabla \cdot \delta \mathrm{P}\right) \cong \nabla \cdot\left(\frac{(\nabla \times \hat{\mathrm{b}})}{B} \delta p_{\|}+\frac{(\hat{\mathrm{b}} \times \nabla B)}{B^{2}} \delta p_{\perp}+\frac{\hat{\mathrm{b}} \hat{\mathrm{b}} \cdot \nabla \times \hat{\mathrm{b}}}{B}\left(\delta p_{\perp}-\delta p_{\|}\right)\right]
$$


On the other hand, using $\mathbf{v}_{D}=\left(v_{\|} / \omega_{c}\right) \nabla \times\left(\hat{\mathbf{b}} v_{\|}\right)$where the gradient is performed with the energy and magnetic moment held fixed, we find the exact relationship

$$
e \int d^{3} v \mathbf{v}_{D} \cdot \nabla \delta f=c \nabla \cdot\left[\frac{(\nabla \times \hat{\mathrm{b}})}{B} \delta p_{\|}+\frac{(\hat{\mathrm{b}} \times \nabla B)}{B^{2}} \delta p_{\perp}\right] .
$$

Hence, neglecting the parallel gradient in the last term on the right-hand side of Eq. (A5) by virtue of our large-aspect-ratio ordering, we obtain Eq. (8) of the text, which relates the macroscopic pressure perturbation to a kinetic integral over the perturbed distribution function. A sum over particle species is implicitly understood on the left-hand side of Eq. (A6), although in this appendix we will focus on the kinetic response of the alpha particles.

We first consider the equilibrium distribution function $F$, which satisfies the steady-state. zero-electric-field form of Eq. (3), viz.,

$$
\left(v_{\|} \hat{\mathbf{b}} \cdot \nabla+\mathbf{v}_{D} \cdot \nabla\right) F=0
$$

For small gyroradius, the solution for $F$ can be expanded as $F=F_{0}+F_{1}$, where $\hat{\mathrm{b}} \cdot \nabla F_{0}=0$, so that $F_{0}=F_{0}(\psi, \Sigma, \mu)$ has spatial dependence only through the poloidal flux $\psi$, and where $F_{1}$ satisfies the equation

$$
v_{\|} \hat{\mathrm{b}} \cdot \nabla F_{1}+\mathrm{v}_{D} \cdot \nabla F_{0}=0
$$

The importance of the toroidal correction $F_{1}$ has been pointed out in Ref. 11, where it was shown how, in large-aspect-ratio tokamak geometry, Eq. (A8) can be integrated for a low-beta plasma to give

$$
F_{1}=-\frac{v_{D 0}}{v_{\|}} q R_{0} \frac{\partial F_{0}}{\partial r}, \quad \text { with } \quad v_{D 0}=\frac{\left(v_{\|}^{2}+\mu B_{0}\right)}{\omega_{c} R_{0}} .
$$

Actually. Eq. (A8) has an exact solution. Introduce flux coordinates $(\psi, \theta, \zeta)$, in terms of which the magnetic field is given as $B=\nabla \zeta \times \nabla \psi+(G / \sigma) \nabla \zeta$, where $\zeta$ is the toroidal angle and $v$ the poloidal flux; recall that in an anisotropic plasma, the quantity $G(\psi)=\sigma R B_{t}$ is a flux function with $\sigma=1+4 \pi\left(p_{\perp}-p_{\|}\right) / B^{2}$. In these coordinates, since $\mathbf{B} \cdot \nabla F_{1}=J^{-1}\left(\partial F_{1} / \partial \theta\right)$. 
with $J=(\mathbf{B} \cdot \nabla \theta)^{-1}=[\nabla \psi \times \nabla \theta \cdot \nabla \zeta]^{-1}$ the Jacobian, and since $\nabla \cdot\left[v_{\|}\left(\hat{\mathbf{b}} \times \nabla F_{0}\right]=\right.$ $J^{-1}(\partial / \partial \theta)\left[\left(v_{\|} G(\psi) / \sigma B\right)\left(\partial F_{0} / \partial \psi\right)\right]$, we can immediately integrate Eq. (A8) to obtain the solution:

$$
F_{1}=-\left(\frac{M c}{e}\right) G(\psi)\left[\frac{v_{\|}}{\sigma B}-\frac{\oint_{0}^{2 \pi} J d \theta\left(\frac{1}{\sigma}\right)}{\oint_{0}^{2 \pi} J d \theta\left(\frac{B}{v_{\|}}\right)}\right]\left(\frac{\partial F_{0}}{\partial \psi}\right) .
$$

In Eq. (A10), the constant (with respect to $\theta$ ) of the integration has been determined in terms of flux surface averages from the condition that $F_{1}$ contribute to no alpha particle accumulation on any flux surface. (Stated more fully, the alpha particle fusion source depends only on flux surface, not on poloidal angle. Because the alpha particles that can reach and thus are counted on a given flux surface are, to lowest order and for given $E, \mu$, and sign of $v_{\|}$, born with equal probability on equidistant inner and outer flux surfaces, the change of the flux-surface averaged alpha particle distribution function due to finite-orbit width effects must be second order. Hence the poloidal average of the first-order change, $F_{1}$, must be zero.) In the limit of low beta and large aspect ratio, with the use of Eq. (21), it is clear that Eq. (A10) reduces to Eq. (A9). The form of Eq. (A10) shows that the total equilibrium distribution function, $F_{0}+F_{1}$, is a function of the toroidal canonical angular momentum $p_{\zeta} \propto \psi-(M c / e) R v_{\|}$; hence the part designated $F_{1}$ describes radial excursion and finite banana width effects.

Next, the solution for the perturbed distribution function is required. Linearize the drift kinetic equation of Eq. (3) with $f=F+\delta f, \mathbf{E}=\delta \mathbf{E}$, and $\mathbf{B}=\mathbf{B}_{0}+\delta \mathbf{B}$ (henceforth we suppress the subscript on the equilibrium magnetic field), where the perturbed magnetic field $\delta \mathbf{B}$ is related to the perturbed electric field $\delta \mathbf{E}$ by Ampére's law as

$$
\begin{aligned}
& i \omega \delta \mathbf{B}=c \nabla \times\left(\hat{\mathbf{b}} \delta E_{\|}+\delta \mathbf{E}_{\perp}\right) \\
& =\left(\delta \mathbf{v}_{E} \cdot \nabla\right) \mathbf{B}+\mathbf{B}\left(\nabla \cdot \delta \mathbf{v}_{E}\right)-(\mathbf{B} \cdot \nabla) \delta \mathbf{v}_{E}-\hat{\mathbf{b}} \times \nabla \delta E_{\|}+\delta E_{\|}(\nabla \times \hat{\mathbf{b}}) .
\end{aligned}
$$


Using the vector identity

$$
\mathbf{v}_{D} \cdot \nabla\left(\delta \mathbf{v}_{E} \cdot \nabla F\right)-\delta \mathbf{v}_{E} \cdot \nabla\left(\mathbf{v}_{D} \cdot \nabla F\right)=\left[\nabla \times\left(\delta \mathbf{v}_{E} \times \mathbf{v}_{D}\right)-\delta \mathbf{v}_{E}\left(\nabla \cdot \mathbf{v}_{D}\right)+\mathbf{v}_{D}\left(\nabla \cdot \delta \mathbf{v}_{E}\right)\right] \cdot \nabla F
$$

we can write the linearized drift kinetic equation in the form

$$
\left(-i \omega+v_{\|} \hat{\mathbf{b}} \cdot \nabla+\mathbf{v}_{D} \cdot \nabla\right) \delta h+\frac{e}{M} \frac{\partial F_{0}}{\partial \varepsilon}\left(1-\frac{\omega_{*}}{\omega}\right)\left(v_{\|} \delta E_{\|}+\mathbf{v}_{D} \cdot \delta \mathbf{E}_{\perp}\right)+R=0
$$

where $\delta h=\delta f-\delta \mathbf{v}_{E} \cdot \nabla F_{0} / i \omega$ and where the diamagnetic frequency $\omega_{*}$ is defined as

$$
\omega_{*}=i\left(\frac{M c}{e B}\right)\left(\frac{\partial F_{0} / \partial \psi}{\partial F_{0} / \partial \varepsilon}\right)(\hat{\mathbf{b}} \times \nabla \psi) \cdot \nabla .
$$

Equation (A8) was used in the derivation of Eq. (A13), and, as a consequence, the gradient operator in $\omega_{*}$ does not operate only on the electric field. $\delta E_{\perp}$, in Eq. (A13) - contrary to the assertion in Ref. $2 \tau$ - but also on the drift velocity $v_{D}$. The quantity $R$ in Eq. (A13) consists of several terms:

$$
\begin{aligned}
R & =\frac{1}{i \omega}\left\{\left(\mathbf{v}_{D} \cdot \delta \mathbf{E}_{\perp}\right)\left[\left(\nabla \times \frac{\hat{\mathrm{b}}}{B}\right) \cdot \nabla F_{0}\right]-\left(\nabla \cdot \mathrm{v}_{D}\right)\left(\delta \mathbf{v}_{E} \cdot \nabla F_{0}\right)\right. \\
& \left.+\left[\left(\frac{\mu \delta B_{\|}}{v_{\|}^{2}}\right)-\left(\delta \mathrm{v}_{E} \cdot \nabla\right) \ln \left(\frac{B}{v_{\|}}\right)\right]\left(\mathrm{v}_{D} \cdot \nabla F_{0}\right)+\left(\delta \mathbf{v}_{D} \cdot \nabla F_{0}\right)\right\}
\end{aligned}
$$

If we adopt the frequency ordering $\omega \sim v_{\|} \hat{\mathrm{b}} \cdot \nabla \gg \mathrm{v}_{D} \cdot \nabla$, then all the terms in $R$ are small, on the order of $\left|v_{D} \cdot \nabla\right| / \omega \ll 1$, and may be neglected in Eq. (A13). Finally, go to the large-aspect-ratio limit, for which $v_{\|}$can be taken to be approximately constant and for which, with the assumption of low beta, we have

$$
\mathrm{v}_{D} \cong-v_{D 0}(\hat{\mathrm{r}} \sin \theta+\hat{\theta} \cos \theta), \quad \text { with } \quad v_{D 0}=\frac{\left(v_{\|}^{2}+\frac{1}{2} v_{\perp}^{2}\right)}{R_{0} \omega_{c}}
$$

In this limit, we introduce the Fourier representation $\delta h(r, \theta, \zeta, t)=\sum_{m} \delta h_{m}(r) \exp [i(-m \theta+$ $n \zeta-\omega t)]$ and obtain the solution for the poloidal components $\delta h_{m}$ of the perturbed distri- 
bution function as follows:

$$
\begin{gathered}
{\left[\omega-\frac{v_{\|}}{R_{0}}\left(-\frac{m}{q(r)}+n\right)\right] \delta h_{m}=\frac{e v_{D 0}}{2 M}\left(\frac{\partial F_{0}}{\partial \varepsilon}-\frac{m}{\omega \omega_{c}} \frac{\partial F_{0}}{r \partial r}\right)\left[\left(\frac{\partial}{\partial r}-\frac{m-1}{r}\right) \delta \Phi_{m-1}\right.} \\
\left.-\left(\frac{\partial}{\partial r}+\frac{m+1}{r}\right) \delta \Phi_{m+1}\right] .
\end{gathered}
$$

Now use this solution for the perturbed distribution function to evaluate the kinetic contribution to the quadratic form, viz., the second term on the right-hand side of Eq. (57):

$$
\begin{gathered}
i \sum_{m} \int_{0}^{a} d r E_{m}^{*}(\omega) L_{k} E_{m}=-\frac{4 \pi i \omega e_{\alpha}}{c^{2}} \int \frac{d^{3} r}{4 \pi^{2} R_{0}} \int d^{3} v \delta f \mathbf{v}_{D} \cdot \delta \mathbf{E}^{*} \\
=\frac{2 \pi \omega e_{\alpha}}{c^{2}} \sum_{m} \int_{0}^{a} r d r \int d^{3} v v_{D 0} \delta f_{m}\left[\left(\frac{\partial}{\partial r}-\frac{m-1}{r}\right) \delta \Phi_{m-1}^{*}-\left(\frac{\partial}{\partial r}+\frac{m+1}{r}\right) \delta \Phi_{m+1}^{*}\right] \\
\cong i\left(\frac{\pi^{2} \omega e_{\alpha}^{2}}{M_{\alpha} c^{2}}\right) \sum_{m} \int_{0}^{a} r d r \int d^{3} v v_{D 0}^{2} W_{m}
\end{gathered}
$$

with

$$
\begin{aligned}
W_{m} & =\left(\frac{\partial F_{0}}{\partial \varepsilon}-\frac{(m+1)}{\omega \omega_{c \alpha}} \frac{\partial F_{0}}{r \partial r}\right)\left|\frac{\partial}{\partial r}\left(r E_{m}\right)-m E_{m}\right|^{2} \delta\left[\omega-\frac{v_{\|}}{R_{0}}\left(-\frac{m+1}{q(r)}+n\right)\right] \\
& +\left(\frac{\partial F_{0}}{\partial \varepsilon}-\frac{(m-1)}{\omega \omega_{c \alpha}} \frac{\partial F_{0}}{r \partial r}\right)\left|\frac{\partial}{\partial r}\left(r E_{m}\right)+m E_{m}\right|^{2} \delta\left[\omega-\frac{v_{\|}}{R_{0}}\left(-\frac{m-1}{q(r)}+n\right)\right]
\end{aligned}
$$

In the last step of Eq. (A18), we dropped the coupling to the $m \pm 2$ Fourier components: kept only the resonant part of the kinetic integral, which will contribute to growth or damping of the wave: and replaced $\delta f$ by $\delta h$, since the difference does not participate in the wave-particle resonance. Equations (A18) and (A19) agree with the result of Ref. 11 when $\omega \ll \omega_{*_{\alpha}}$.

For the alpha particle distribution function $F_{0}$ in Eq. (A18), we will use the classical steady-state "slowing down" distribution, ${ }^{43}$ isotropic in pitch angle, which is given by

$$
F_{0}(r, v)=\frac{S(r) \tau_{s}}{4 \pi\left(v^{3}+v_{c}^{3}\right)} H\left(v_{\alpha 0}-v\right) .
$$


Here $v_{\alpha 0}=\left(2 \mathcal{E}_{\alpha 0} / M_{\alpha}\right)^{1 / 2}$ is the velocity that corresponds to the alpha particle birth energy $\mathcal{E}_{\alpha 0}$, and $H\left(v_{\alpha 0}-v\right)$ is the Heaviside function. The velocity $v_{c}$ is given by

$$
v_{c}=\left[\frac{3 \sqrt{\pi}}{4}\left(\frac{M_{e}}{M_{p}}\right) \frac{1}{N_{e} \ln \Lambda_{e}} \sum_{i} \frac{Z_{i}^{2} N_{i} \ln \Lambda_{i}}{A_{i}}\right]^{1 / 3} v_{e}
$$

with $v_{e}=\left(2 T_{e} / M_{e}\right)^{1 / 2}$ the electron thermal velocity, $M_{e}$ the electron mass, $M_{p}$ the proton mass, $A_{i}$ the ion atomic number, $Z_{i}$ the ion charge state, $N_{i}$ and $N_{e}$ the ion and electron densities, and the Coulomb logarithms

$$
\begin{gathered}
\ln \Lambda_{e}=23.9+\ln \left(\frac{T_{e}}{\sqrt{N}_{e}}\right) \\
\ln \Lambda_{i}=14.2+\ln \left[\left(\frac{T_{e}}{N_{e}}\right)^{1 / 2} \frac{A_{\alpha} A_{i} v_{\alpha 0}}{\left(A_{\alpha}+A_{i}\right)}\right]
\end{gathered}
$$

with $T_{e}$ in units of $e V$ and $N_{e}$ in $\mathrm{cm}^{-3}$. The slowing-down time $\tau_{s}$ is given by

$$
\tau_{s}=\frac{3 M_{e} M_{\alpha} v_{e}^{3}}{16 \sqrt{\pi} Z_{\alpha}^{2} e^{4} N_{e} \ln \Lambda_{e}}=6.30 \times 10^{8} \frac{T_{e}^{3 / 2}}{N_{e} \ln \Lambda_{e}}\left(\text { for } Z_{\alpha}=2\right)
$$

and can be rewritten in terms of the velocity $v_{c}$ as follows:

$$
\tau_{s}^{-1}=\sum_{i} \frac{4 \pi e_{\alpha}^{2} e_{i}^{2} N_{i} \ln \Lambda_{i}}{M_{i} M_{\alpha} r_{c}^{3}}
$$

Note that the number density of the slowing-down, non-thermalized alpha particles is given by

$$
N_{\alpha}(r)=\int_{0}^{\infty} d^{3} v F_{0}(r, v)=\frac{S \tau_{s}}{3} \ln \left[1+\left(\frac{v_{\alpha 0}}{v_{c}}\right)^{3}\right] \cong S \tau_{s} \ln \left(\frac{v_{\alpha 0}}{v_{c}}\right) .
$$

For an equal mixture D-T ignition plasma (for which $\mathcal{E}_{\alpha 0}=3.5 \mathrm{MeV}$ and hence $v_{\alpha 0}=$ $1.3 \times 10^{9} \mathrm{~cm} / \mathrm{s}$ ), the alpha particle source rate $S$ is given by $S=N_{D} N_{T}\langle\sigma v\rangle_{D T}$, with $\langle\sigma v\rangle_{D T}$ the reaction rate ${ }^{44}$ for the production of alpha particles and $N_{D}$ and $N_{T}$ the deuterium and tritium densities. Some useful values for the reaction rate ${ }^{44}\langle\sigma v\rangle_{D T}=1.1 \times 10^{-16} \mathrm{~cm}^{3} / \mathrm{s}$ for $T_{i}=10 \mathrm{keV}, 2.6 \times 10^{-16} \mathrm{~cm}^{3} / \mathrm{s}$ for $T_{i}=15 \mathrm{keV}$, and $4.2 \times 10^{-16} \mathrm{~cm}^{3} / \mathrm{s}$ for $T_{i}=20 \mathrm{keV}$. Also, when $T_{e} \cdot=10 \mathrm{keV}$ and $N_{e}=10^{14} \mathrm{~cm}^{-3}$, we find $\ln \Lambda_{e}=17, \ln \Lambda_{D}=\ln \Lambda_{T}=24$, 
$v_{e}=5.9 \times 10^{9} \mathrm{~cm} / \mathrm{s}, v_{c}=4.5 \times 10^{8} \mathrm{~cm} / \mathrm{s}$, and $\tau_{s}=0.37 \mathrm{~s}$. For the sake of comparison, when $B=5 \mathrm{~T}$, we have $v_{A}=6.9 \times 10^{8} \mathrm{~cm} / \mathrm{s}$.

Let us now approximately evaluate the alpha particle-induced growth rate from Eq. (A18) for the TAE mode with $n=1$ that couples $m=1$ and $m=2$, using the slowing-down distribution function of Eq. (A20). We simplify Eq. (A18) by assuming that the major contribution to the radial integral arises from the vicinity of the gap region, at $q=3 / 2$, where $\left|\partial E_{m} / \partial r\right|$ is peaked, and by evaluating all slowly varying quantities at the gap location $r=r_{g}$, such as the Alfvén velocity $v_{A}(r) \rightarrow v_{A}\left(r_{g}\right) \equiv v_{A g}$ for short. In the same spirit. we take $\omega \approx v_{A g} / 3 R_{0}$, which is the frequency at which $\left|k_{\|, m=1}\right|^{2}=\left|k_{\|, m=2}\right|^{2}$, where the cylindrical continua cross each other. Then, we note that the $m=1$ mode contributes to the wave-particle resonance delta functions in Eq. (A19) when either $v_{\|}=-v_{A g}$ or $v_{\|}=v_{A g} / 3$, whereas the $m=2$ mode resonates when $v_{\|}=v_{A_{g}}$ or $v_{\|}=-v_{A_{g}} / 3$. For simplicity, we will neglect the lower velocity resonances at $v_{\|}= \pm v_{A g} / 3$, since when $v_{\alpha 0}>v_{A g}$, the particles at. velocities $\left|v_{\|}\right| \approx v_{A g} / 3$ tend to be toroidally trapped, whereas Eq. (A19) accounts for only' the circulating particle effects. Now, define a measure of the alpha particle beta value as follows,

$$
\tilde{\beta}_{\alpha}=\frac{4 \pi M_{\alpha} v_{\alpha 0}^{2} S \tau_{s}}{3 B_{0}^{2}}=\frac{\beta}{3}\left(\frac{M_{\alpha} v_{\alpha 0}^{2}}{2\left(T_{e}+T_{i}\right)}\right) \frac{N_{D} N_{T}}{N_{e}^{2}} N_{e} \tau_{s}\langle\sigma v\rangle
$$

where $\beta=8 \pi N_{e}\left(T_{e}+T_{i}\right) / B_{0}^{2}$ is the beta of the background plasma at $r=r_{3}$. Note that when $v_{\alpha 0} \gg v_{\varepsilon}, \tilde{\beta}_{\alpha}$ approximates the exact expression for the alpha particle beta value.

$$
\beta_{\alpha}=\frac{8 \pi}{3 B_{0}^{2}} \int_{0}^{\infty} d^{3} v M_{\alpha} v^{2} F_{0}(r, v)
$$

Also. define the alpha particle diamagnetic frequency in the same limit as follows:

$$
\tilde{\omega}_{* \alpha}=\frac{v_{\alpha 0}^{2}}{2 r \omega_{c \alpha}}\left|\frac{\partial}{\partial r}\left(\ln \tilde{\beta}_{\alpha}\right)\right|
$$

Then, with the simplifications noted previously, we reduce Eq. (A19) to the following form:

$$
i \sum_{m} \int d r E_{m}^{*}\left(\omega^{\prime}\right) L_{k} E_{m}=i \frac{9 \pi}{4} \frac{\omega}{v_{\alpha 0} R_{0}} \int_{0}^{a} d r r^{3} \tilde{\beta}_{\alpha}\left\{-P_{\alpha}\left(\left|\frac{\partial E_{1}}{\partial r}\right|^{2}+\left|\frac{\partial E_{2}}{\partial r}+\frac{3 E_{2}}{r}\right|^{2}\right)\right. \text {. }
$$




$$
\left.+2\left(\frac{\tilde{\omega}_{*_{\alpha}}}{\omega}\right) Q_{\alpha}\left(2\left|\frac{\partial E_{1}}{\partial r}\right|^{2}+\left|\frac{\partial E_{2}}{\partial r}+\frac{3 E_{2}}{r}\right|^{2}\right)\right\} .
$$

The velocity integrals $P_{\alpha}$ and $Q_{\alpha}$ are given. by

$$
\begin{aligned}
P_{\alpha}= & \frac{1}{v_{\alpha 0} S \tau_{s}} \int_{0}^{\infty} d^{3} v\left(\frac{1}{2} v_{\perp}^{2}+v_{\|}^{2}\right)^{2} \delta\left(\left|v_{\|}\right|-v_{A g}\right)\left(-\frac{\partial F_{0}}{v \partial v}\right) \\
= & \frac{1}{8}\left[\frac{\left(1+x_{A}^{2}\right)^{2}}{1+x_{c}^{3}}+3 \int_{x_{A}}^{1} d x \frac{x^{6}\left(1+\frac{x_{A}^{2}}{x^{2}}\right)^{2}}{\left(x^{3}+x_{c}^{3}\right)^{2}}\right] H\left(1-x_{A}\right) \\
= & \frac{1}{2}\left[\frac{x_{A}^{4}}{x_{A}^{3}+x_{c}^{3}}+1-x_{A}-x_{c}^{3} I_{0}+x_{A}^{2} I_{1}\right] H\left(1-x_{A}\right) \\
& \longrightarrow \frac{1}{2}\left[1+x_{A}\left(1-x_{A}\right)\right] H\left(1-x_{A}\right) \text { for } x_{c}^{3} \ll x_{A}^{3}
\end{aligned}
$$

and

$$
\begin{aligned}
Q_{\alpha}= & \frac{1}{v_{\alpha 0}^{3} S \tau_{s}} \int_{0}^{\infty} d^{3} v\left(\frac{1}{2} v_{\perp}^{2}+v_{\|}^{2}\right)^{2} \delta\left(\left|v_{\|}\right|-v_{A g}\right) F_{0} \\
= & \frac{1}{8} H\left(1-x_{A}\right) \int_{x_{A}}^{1} d x \frac{x^{5}\left(1+\frac{x_{A}^{2}}{x^{2}}\right)^{2}}{x^{3}+x_{c}^{3}} \\
= & \frac{1}{24}\left[1-x_{A}^{3}+6 x_{A}^{2}\left(1-x_{A}\right)-x_{c}^{3} \ln \left(\frac{1+x_{c}^{3}}{x_{A}^{3}+x_{c}^{3}}\right)-6 x_{c}^{2} x_{A}^{2} I_{0}+3 x_{A}^{4} I_{1}\right] H\left(1-x_{A}\right) \\
& \longrightarrow \frac{1}{24}\left[1+x_{A}^{2}\left(6-4 x_{A}-3 x_{A}^{2}\right)\right] H\left(1-x_{A}\right) \text { for } x_{c}^{3} \ll x_{A}^{3}
\end{aligned}
$$

with $H(\xi)$ the Heaviside step function and with

$$
\begin{gathered}
I_{\ell=0,1}=\int_{x_{A}}^{1} d x \frac{x^{\ell}}{x^{3}+x_{c}^{3}} \\
=\frac{x_{c}^{\ell-2}}{3}\left\{\sqrt{3} \arctan \left[\frac{2 \sqrt{3} x_{c}\left(1-x_{A}\right)}{3 x_{c}^{2}+\left(2-x_{c}\right)\left(2 x_{A}-x_{c}\right)}\right]+\frac{1}{2}(-1)^{\ell} \ln \left[\left(\frac{1+x_{c}}{x_{A}+x_{c}}\right)^{2}\left(\frac{x_{A}^{2}-x_{A} x_{c}+x^{2}}{1-x_{c}+x_{c}^{2}}\right)\right]\right\} \\
\longrightarrow 2^{\ell-1}\left(x_{A}^{\ell-2}-1\right) \quad \text { for } x_{c}^{3} \ll x_{A}^{3}
\end{gathered}
$$


and $x=v / v_{\alpha 0}, x_{A}=v_{A} / v_{\alpha 0}$ and $x_{c}=v_{c} / v_{\alpha 0}$. Finally, we insert the result of Eq. (A29) into Eq. (63), neglecting the continuum damping term on the right-hand side of Eq. (63), which has already been perturbatively evaluated in Sec. IV. We use the peakedness approximation $\left|\partial E_{m} / \partial r\right| \gg\left|E_{m} / r\right|$ and also use Eqs. (80) and (83) to obtain the following result for the alpha-particle-induced growth rate of the $n=1, m=1 / m=2$ TAE mode:

$$
\begin{aligned}
& \frac{\gamma_{\text {alpha }}}{\omega} \cong\left(\frac{9 \pi}{8}\right) \tilde{\beta}_{\alpha}\left[-3 x_{A} P_{\alpha}+10 x_{A}\left(\frac{\tilde{\omega}_{* \alpha}}{\omega}\right) Q_{\alpha}\right] \\
& \longrightarrow\left(\frac{2 \tau \pi}{16}\right) \tilde{\beta}_{\alpha} x_{A} H\left(1-x_{A}\right)\left\{-\left[1+x_{A}\left(1-x_{A}\right)\right]+\frac{5}{18}\left[1+x_{A}^{2}\left(6-4 x_{A}-3 x_{A}^{2}\right)\right] \frac{\tilde{\omega}_{*_{\alpha}}}{\omega^{\prime}}\right\}
\end{aligned}
$$

for $x_{c}^{3} \ll x_{A}^{3}$. Recall that $\tilde{3}_{\alpha}$ can be exprcssed in terms of local plasma parameters given by Eq. (A26). Plots of the functions $3 x_{A} P_{\alpha}\left(x_{A}\right)$ and $10 x_{A} Q_{\alpha}\left(x_{A}\right)$ are given in Figs. 12(a) and $12(b)$. 


\section{Appendix B: Proof of the Positivity of the Continuum Damping Rate}

In order to show from Eq. (63) that the Alfvén continuum resonance always leads to a damping contribution, since we already know from Eq. $(60)$ that $\omega_{0}^{-1} \partial G\left(\omega_{0}, E_{m}^{(0)}\right) / \partial \omega_{0}>0$ holds, it will suffice to prove the following inequality:

$$
\frac{1}{\omega} \frac{\partial\left\|D\left(\omega, r_{s, j}\right)\right\|}{\partial \omega} \sum_{m, n} D^{m n}\left(\omega, r_{s, j}\right) C_{m}^{(0)}\left(r_{s, j}\right) C_{n}^{(0)}\left(r_{s, j}\right)>0 .
$$

The proof is as follows. Consider the following quantity:

$$
K(\omega, r)=\sum_{n, m} A_{m}(r) \frac{\partial}{\partial \omega}\left[\frac{D_{m n}(\omega, r)}{\omega}\right] A_{n}(r)
$$

for arbitrary real. nonsingular functions $A_{m}(r)$ and arbitrary real frequency $w$. with $D_{m n}$ the matrix elements defined in Eq. (53). Since $K(\omega, r)$ can be explicitly written in quadratic form as

$$
K^{\prime}(\omega \cdot r)=\oint_{0}^{2 \pi} \frac{d \theta}{2 \pi}\left\{\frac{1}{v_{A}^{2}}(1+2 \hat{\xi} \cos \theta)\left[\sum_{m} A_{m} \exp (-i m \theta)\right]^{2}+\sum_{m} \frac{k_{\| m}^{2}}{\omega^{2}}\left(. A_{m}\right)^{2}\right\}
$$

we immediately observe that $K^{\prime}(\omega, r)$ is positive definite for $\hat{\varepsilon}<1 / 2$.

For the functions $A_{m}(r)$, it is allowable to take the specific choice of

$$
A_{m}(r)=\sum_{p} D^{m p}(\omega \cdot r) C_{p}^{(0)}(r)
$$

where $\omega$ is the real, discrete eigenfrequency of a TAE mode and the corresponding "flux" functions $C_{p}^{(0)}(r)$ are related to the real TAE radial eigenfunctions through Eq. (52). Here $D^{m p}$ denotes the cofactor matrix elements, which satisfy Eq. (5.5). We proceed to substitute Eq. (B4) into Eq. (B2) and then manipulate the expression for $K(\omega, r)$ as follows:

$$
\begin{gathered}
0 \leq K(\omega, r)=\sum_{m, n} A_{m}(r) \frac{\partial}{\partial \omega}\left[\frac{D_{m n}(\omega, r)}{\omega}\right] A_{n}(r) \\
=\sum_{m, n} \sum_{p, q}\left[D^{m p}(\omega, r) C_{p}^{(0)}(r)\right]\left[D^{n q}(\omega \cdot r) C_{q}^{(0)}(r)\right] \frac{\partial}{\partial \omega}\left[\frac{D_{m n}(\omega, r)}{\omega}\right]
\end{gathered}
$$




$$
\begin{gathered}
=\sum_{m, n} \sum_{p, q} D^{m p}(\omega, r) C_{p}^{(0)}(r) C_{q}^{(0)}(r)\left\{\frac{\partial}{\partial \omega}\left[\frac{D_{m n}(\omega, r) D^{n q}(\omega, r)}{\omega}\right]-\frac{D_{m n}(\omega, r)}{\omega} \frac{\partial}{\partial \omega}\left[D^{n q}(\omega, r)\right]\right\} \\
=\frac{\partial}{\partial \omega}\left[\frac{\|D(\omega, r)\|}{\omega}\right] \sum_{p, q} D^{q p}(\omega, r) C_{p}^{(0)}(r) C_{q}^{(0)}(r)-\frac{\|D(\omega, r)\|}{\omega} \sum_{p, q}\left[\frac{\partial}{\partial \omega} D^{q p}(\omega, r)\right] C_{p}^{(0)}(r) C_{q}^{(0)}(r) \\
=\left[\frac{1}{\omega} \frac{\partial}{\partial \omega}\|D(\omega, r)\|\right] \sum_{p, q} D^{q p}(\omega, r) C_{q}^{(0)}(r) C_{q}^{(0)}(r) \\
-\frac{\|D(\omega, r)\|}{\omega_{0}^{2}} \sum_{p, q}\left\{\frac{\partial}{\partial \omega}\left[\omega D^{q \rho}(\omega, r)\right]\right\} C_{q}^{(0)}(r) C_{p}^{(0)}(r) .
\end{gathered}
$$

In the preceding, we have used the symmetry of the matrix elements $\left(D_{m n}=D_{n m}\right)$ and contracted the $D_{m n}$ and cofactor $D^{p q}$ matrix elements in accordance with Eq. (54), with $\|D(\omega, r)\|$ being the determinant of the matrix whose elements are $D_{m n}$.

Finally, we evaluate the final expression for $K(\omega, r)$ given on the last line of Eq. (B5). at any continuum resonance point $r=r_{s, j}(\omega)$ for the frequency $\omega$. At these points the determinant vanishes by definition: $\left\|D\left(\omega, r_{s, j}\right)\right\|=0$. Consequently the second term on the last line of Eq. (B5) disappears. whereas the first term when evaluated at $r=r_{s, j}$ is nothing more than the quantity on the left-hand side of Eq. (B1). The inequality in Eq. (B1) is thus demonstrated, which establisices our assertion that Alfvén continuum resonances always contribute to wave damping. 


\section{Appendix C: Evaluation of $\partial G / \partial \omega$}

Here, a method is described for modifying the frequency derivative of the principal value function $G\left(\omega, E_{m}\right)$, so that the apparently divergent integral in Eq. (95) is avoided. The result for the case without toroidal coupling is explicitly presented, and the appropriate generalization to the case with toroidal coupling is indicated. In particular, a useful form for the three-mode coupling problem is given.

The essence of this improved treatment is the retention of higher-order terms in the discontinuity in the wave function at each continuum resonance point. To illustrate the method, we neglect toroidal coupling and assume the existence of only one continuum resonance point, at $r=r_{s}$, where $\omega^{2}=k_{\|}^{2}\left(r_{s}\right) v_{A}^{2}\left(r_{s}\right)$ is satisfied. From Eq. (39) without the alpha particle kinetic destabilization term $L_{k}$, the quadratic form for the cylindrical problem is given by the following expression:

$$
\begin{gathered}
\left(\int_{0}^{r_{s}-\eta_{1}} d r+\int_{\tau_{s}+\eta_{2}}^{a} d r\right)\left\{\left(\frac{\omega^{2}}{v_{A}^{2}}-k_{\| m}^{2}\right)\left[r^{3}\left(\frac{d E_{m}}{d r}\right)^{2}+r\left(m^{2}-1\right) E_{m}^{2}\right]-\omega^{2} r^{2} E_{m}^{2} \frac{d}{d r}\left(\frac{1}{v_{A}^{2}}\right)\right\} \\
=-\left[E_{m}\left(r_{s}+\eta_{2}\right)-E_{m}\left(r_{s}-\eta_{1}\right)\right] C_{m}\left(r_{s}\right)+E_{m}(a) C_{m}(a)
\end{gathered}
$$

in the limit where $\eta_{1}$ and $\eta_{2} \rightarrow 0$, but with the ratio $\eta_{1} / \eta_{2}$ arbitrary. The expression on the left-hand side of Eq. (C1) is the quantity $G\left(\omega, E_{m}\right)$ that was previously defined in Eq. (42) for the case when $\eta_{1}=\eta_{2}$, in which case the standard definition of the principal value was employed. However, we note that it is not necessary to have $\eta_{1}=\eta_{2}$ in order for $G\left(\omega, E_{m}\right)$ to be well defined, and indeed in the discussion that follows it will be convenient to exploit this freedom. The eigermode $E_{m}$ obeys the boundary conditions that $E_{m}(r=0)$ is regular, that $E_{m}(r=a)=0$, and that $C_{m}(r)$ is continuous at the resonance location $r=r_{s}$. However $E_{m}(r)$ is discontinuous at the resonance $r_{s}$. In Sec. IV we found, for the case when $\eta_{1}=\eta_{2}=\eta$, that the discontinuity in $E_{m}(r)$ at the resonance $r_{s}$ is determined from causality 
to be

$$
E_{m}\left(r_{s}+\eta\right)-E_{m}\left(r_{s}-\eta\right)=-i \pi \operatorname{sgn}(\omega) \frac{C_{m}\left(r_{s}\right)}{r_{s}^{3}\left|\frac{d}{d r}\left(\frac{\omega^{2}}{v_{A}^{2}}-k_{\| m}^{2}\right)\right|_{r=r_{s}}} .
$$

This result was obtained by integrating the equation for $E_{m}(r)$ in the complex plane from $r_{s}-\eta$ to $r_{s}+\eta$ along a contour on which $\operatorname{Im}\left(\omega / v_{A}^{2}-k_{\| m}^{2} / \omega\right)>0$.

As discussed in Sec. IV, the solution proceeds perturbatively by writing $\omega=\omega_{0}+\delta \omega$ and $E_{m}=E_{m}^{(0)}+\delta E_{m}$. The zeroth-order eigenfrequency $\omega_{0}$ and the lowest-order eigenmode $E_{m}^{(0)}$ are obtained by integrating the mode equation from the magnetic axis to the edge of the plasma, with $E_{m}^{(0)}$ regular at the origin and vanishing at the edge, but with $E_{m}^{(0)}\left(r_{s 0}+\eta_{0}\right)=$ $E_{m}^{(0)}\left(r_{s 0}-\eta_{0}\right)$ for $\eta_{0} / r_{s 0} \ll 1$, where $r=r_{s 0}$ is the resonance location corresponding to the frequency $\omega_{0}$. The jump in the true eigenfunction $E_{m}$ near the resonance point is contained in $\delta E_{m}$. We then evaluate Eq. (C1) for $\omega=\omega_{0}+\delta \omega$ and $E_{m}=E_{m}^{(0)}+\delta E_{m}$, neglecting terms that are quadratic in the perturbation quantities. In the same manner as described in the text, we integrate the terms linear in $\delta E_{m}$ by parts and use the fact that $E_{m}^{(0)}$ satisfies the eigenmode equation for $\omega=\omega_{0}$. We then obtain

$$
\delta \omega^{\prime} \frac{\partial G\left(\omega_{0}, E_{m}^{(0)}\right)}{\delta \omega_{0}}=\left[\delta E_{m}\left(r_{s 0}+\eta_{0}: \omega_{0}+\delta \omega\right)-\delta E_{m}\left(r_{s 0}-\eta_{0}: \omega_{0}+\delta \omega\right)\right] C_{m}^{(0)}\left(r_{s 0}\right)
$$

where the sign of the discontinuity term on the right-hand side of Eq. (C2) has switched sign relative to that on the right-hand side of Eq. (C1). The notation $\delta E_{m}\left(r_{s 0}+\eta_{0} ; \omega_{0}+\delta \omega\right)$ is used to emphasize the implicit frequency dependence of the solution. The quantity $\partial G / \partial \omega_{0}$ is given by the following expression [cf. Eq. (45)]:

$$
\begin{aligned}
& \frac{\partial G\left(\omega_{0}, E_{m}^{(0)}\right)}{\partial \omega_{0}}=\left(\frac{2}{\omega_{0}}\right) \cdot\left(\int_{0}^{r_{s 0}-\eta_{0}} d r+\int_{r_{s 0}+\eta_{0}}^{a} d r\right)\left\{\left(\frac{\omega_{0}^{2}}{v_{A}^{2}}\right)\left[r^{3}\left(\frac{d E_{m}^{(0)}}{d r}\right)^{2}+r\left(m^{2}-1\right)\left(E_{m}^{(0)}\right)^{2}\right]\right. \\
& \left.-\omega_{0}^{2} r^{2}\left(E_{m}^{(0)}\right)^{2} \frac{d}{d r}\left(\frac{1}{v_{A}^{2}}\right)\right\} .
\end{aligned}
$$

Notice that in Eqs. (C3) and (C4), we have exercised the freedom associated with $\eta_{1} \neq \eta_{2}$ by choosing $\eta_{1}=\eta_{0}+\delta \omega\left(\partial r_{s 0} / \partial \omega_{0}\right)$ and $\eta_{2}=\eta_{0}-\delta \omega\left(\partial r_{s 0} / \partial \omega_{0}\right)$. As was pointed out in 
Sec. VI, there is a difficulty in that the quantity $\partial G / \partial \omega_{0}$ of Eq. (C4) is a divergent integral in the limit when $\eta_{0} \rightarrow 0$, since $\left[d E_{m}^{(0)} / d r\right]^{2} \propto\left(r-r_{s 0}\right)^{-2}$ near the resonance point. However, we now show that this apparent divergence is removed when corrections to the discontinuity in the wave function are retained.

Near the resonance point $r=r_{s 0}$, the differential equation for $E_{m}(r)$ is approximately given by

$$
\frac{\omega_{0}^{2} r_{s 0}^{3}}{v_{A}^{2}\left(r_{s 0}\right)} \frac{d}{d r}\left\{\left[\frac{2 \delta \omega}{\omega_{0}}+\left(r-r_{s 0}\right) \frac{v_{A}^{2}\left(r_{s 0}\right)}{\omega_{0}^{2}}\left[\frac{d}{d r}\left(\frac{\omega_{0}^{2}}{v_{A}^{2}}-k_{\| m}^{2}\right)\right]_{r=r_{s 0}}\right] \frac{d E_{m}}{d r}\right\} \cong 0 .
$$

The solution of Eq. (C5) allows a "large" solution that is logarithmically divergent at $r=$ $r_{s} \cong r_{s 0}+\delta \omega\left(\partial r_{s 0} / \partial \omega_{0}\right)$ and also a "small" solution that is constant. Note that $\partial r_{s 0} / \partial \omega_{0}=$ $-\left(2 \omega_{0} / v_{A}^{2}\right)\left[\frac{d}{d r}\left(\frac{\omega_{0}^{2}}{v_{A}^{2}}-k_{\| m}^{2}\right)\right]_{r=r_{s 0}}^{-1}=\left(2 / \omega_{0}\right)\left[\frac{d}{d r} \ln \left(k_{\| m}^{2} v_{A}^{2}\right)\right]_{r=r_{s 0}}^{-1}$. The mix of the two solutions is implicitly determined from satisfying the boundary conditions at $r=0$ and $r=a$. Near $r=r_{s}$, the solutions are specifically given as

$$
E_{m}(r)=\frac{C_{m}\left(r_{s 0}\right)}{r_{s 0}^{3}\left[\frac{d}{d r}\left(\frac{\omega^{2}}{v_{A}^{2}}-k_{\| m}^{2}\right)\right]_{r_{s 0}}}\left\{\ln \left(r-r_{s}\right)+\Delta^{+}\left(\omega_{0}+\delta \omega\right)\right\}, \text { for } r>r_{s}
$$

and

$$
E_{m}(r)=\frac{C_{m}\left(r_{s 0}\right)}{r_{s 0}^{3}\left[\frac{d}{d r}\left(\frac{\omega^{2}}{v_{A}^{2}}-k_{\| m}^{2}\right)\right]_{r_{s 0}}}\left\{\ln \left(r_{s}-r\right)+\Delta^{-}\left(\omega_{0}+\delta \omega\right)\right\}, \text { for } r<r_{s}
$$

By virtue of the construction of the lowest-order eigenfunction $E_{m}^{(0)}$, we have $\Delta^{+}\left(\omega_{0}\right)=$ $\Delta^{-}\left(\omega_{0}\right)$. In Eq. (C.3) we need to evaluate. for $\omega=\omega_{0}+\delta \omega$. the following discontinuity:

$$
\begin{aligned}
& \delta E_{m}\left(r_{s 0}+\eta_{0}\right)-\delta E_{m}\left(r_{s 0}-\eta_{0}\right)=E_{m}\left(r_{s 0}+\eta_{0}\right)-E_{m}\left(r_{s 0}-\eta_{0}\right) \\
& \quad=E_{m}\left(r_{s}+\eta_{0}-\delta \omega \frac{\partial r_{s 0}}{\partial \omega_{0}}\right)-E_{m}\left(r_{s}-\eta_{0}-\delta \omega \frac{\partial r_{s 0}}{\partial \omega_{0}}\right) \\
& \cong\left[E_{m}\left(r_{s}+\eta_{0}\right)-E_{m}\left(r_{s}-\eta_{0}\right)\right]-\delta \omega\left(\frac{\partial r_{s 0}}{\partial \omega_{0}}\right)\left[\frac{\partial E_{m}\left(r_{s}+\eta_{0}\right)}{\partial r}-\frac{\partial E_{m}\left(r_{s}-\eta_{0}\right)}{\partial r}\right](\mathrm{CS})
\end{aligned}
$$


where we assumed $\left|\delta \omega\left(\frac{\partial r_{30}}{\partial \omega_{0}}\right) / \eta_{0}\right| \ll 1$. Then from Eqs. (C2) and (C4), we obtain

$$
\begin{aligned}
& \delta E_{m}\left(r_{s 0}+\eta_{0}\right)-\delta E_{m}\left(r_{s 0}-\eta_{0}\right) \\
& \quad=-i \pi \frac{\operatorname{sgn}\left(\omega_{0}\right) C_{m}^{(0)}\left(r_{s 0}\right)}{r_{s 0}^{3}\left|\frac{d}{d r}\left(\frac{\omega^{2}}{v_{A}^{2}}-k_{\| m}^{2}\right)\right|_{r_{s 0}}}+\frac{4 \delta \omega}{\eta_{0}}\left(\frac{\omega_{0}}{r_{s 0}^{3} v_{A}^{2}\left(r_{s 0}\right)}\right) \frac{C_{m}^{(0)}\left(r_{s 0}\right)}{\left[\frac{d}{d r}\left(\frac{\omega_{0}^{2}}{v_{A}^{2}}-k_{\| m}^{2}\right)\right]_{r_{s 0}}} .
\end{aligned}
$$

The $i \pi$ term in Eq. (C9) has already been exhibited in the text [cf. Eq. (50)], and the term with $4 \delta \omega / \eta_{0}$ is an additional contribution that is needed to cancel the divergence in $\partial G / \partial \omega_{0}$.

When we introduce the result of Eq. (C9) into Eq. (C3), we obtain

$$
\delta \omega \frac{\partial \tilde{G}\left(\omega_{0}, E_{m}^{(0)}\right)}{\partial \omega_{0}}=-i \pi \sum_{j} \operatorname{sgn}\left(\omega_{0}\right) \frac{\left[C_{m}^{(0)}\left(r_{s 0, j}\right)\right]^{2}}{r_{s 0, j}^{3}\left|\frac{d}{d \tau}\left(\frac{\omega_{0}^{2}}{v_{A}^{2}}-k_{\| m}^{2}\right)\right|_{r_{s 0, j}}} .
$$

Equation $(\mathrm{C} 10)$ yields the same result for the continuum damping rate $\gamma=\operatorname{Im}(\delta \omega)$ as that given in Eq. (51) in the text, except that $\partial G / \partial \omega_{0}$ is now replaced by the quantity $\partial \tilde{G} / \partial \omega_{0}$, defined as follows:

$$
\frac{\partial \tilde{G}\left(\omega_{0}, E_{m}^{(0)}\right)}{\partial \omega_{0}}=\frac{\partial G\left(\omega_{0}, E_{m}^{(0)}\right)}{\partial \omega_{0}}-\sum_{j}\left(\frac{4}{\eta_{0 j}}\right)\left(\frac{\omega_{0}}{r_{s 0, j}^{3} v_{A}^{2}\left(r_{s 0, j}\right)}\right) \frac{\left[C_{m}^{(0)}\left(r_{s 0, j}\right)\right]^{2}}{\left[\frac{d}{d r}\left(\frac{\omega_{0}^{2}}{v_{A}^{2}}-k_{\| m}^{2}\right)\right]_{r=r_{s 0, j}}^{2}}
$$

where we have added the possibility of having multiple resonance points $r_{s 0, j}$.

We can readily verify that $\lim _{\eta_{0} \rightarrow 0}\left[\partial \tilde{G}\left(\omega_{0}, E_{m}^{(0)}\right) / \partial \omega_{0}\right]$ is well behaved, albeit no longer intrinsically positive definite. By using the identity

$$
\frac{2}{\eta_{0 j}}=\int_{0}^{r_{s 0}-\eta_{0},} \frac{d r}{\left(r-r_{s 0, j}^{2}\right)}+\int_{r_{s 0}+\eta_{0},}^{a} \frac{d r}{\left(r-r_{s 0, j}\right)^{2}}+\frac{a}{r_{s 0, j}\left(a-r_{s 0, j}\right)}
$$

we can write the quantity $\partial \widetilde{G}\left(\omega_{0}, E_{m}^{(0)}\right) / \partial \omega_{0}$ in the following form:

$$
\begin{aligned}
& \frac{\partial \tilde{G}\left(\omega_{0}, E_{m}^{(0)}\right)}{\partial \omega_{0}}=\frac{2}{\omega_{0}}\left(\int_{0}^{r_{s 0}-\eta_{0}} d r+\int_{r_{s 0}+\eta_{0}}^{a} d r\right)\left\{\left[\left(\frac{\omega_{0}^{2} r^{3}}{v_{A}^{2}}\right)\left(\frac{d E_{m}^{(0)}}{d r}\right)^{2}\right.\right. \\
& \left.\quad-\sum_{j} \frac{1}{\left(r-r_{s 0, j}\right)^{2}}\left(\frac{\omega_{0}}{r_{s 0, j}^{3} v_{A}^{2}\left(r_{s 0, j}\right)}\right) \frac{\left[C_{m}^{(0)}\left(r_{s 0, j}\right)\right]^{2}}{\left[\frac{d}{d r}\left(\frac{\omega_{0}^{2}}{v_{A}^{2}}-k_{\| m}^{2}\right)\right]_{r s 0, j}^{2}}\right]
\end{aligned}
$$




$$
\begin{aligned}
& \left.+r\left(\frac{\omega_{0}^{2}}{v_{A}^{2}}\right)\left(m^{2}-1\right)\left(E_{m}^{(0)}\right)^{2}-\omega_{0}^{2} r^{2}\left(E_{m}^{(0)}\right)^{2} \frac{d}{d r}\left(\frac{1}{v_{A}^{2}}\right)\right\} \\
& -\sum_{j} \frac{2 a}{r_{s 0, j}\left(a-r_{s 0, j}\right)}\left(\frac{\omega_{0}}{r_{s 0, j}^{3} v_{A}^{2}\left(r_{s 0, j}\right)}\right) \frac{\left[C_{m}^{(0)}\left(r_{s 0, j}\right)\right]^{2}}{\left[\frac{d}{d r}\left(\frac{\omega_{0}^{2}}{v_{A}^{2}}-k_{\| m}^{2}\right)\right]_{r s, j}^{2}} .
\end{aligned}
$$

In Eq. (C13), note that the $\left(r-r_{s 0, j}\right)^{-2}$ divergence arising from the $\left[d E_{m}^{(0)} / d r\right]^{2}$ term in the integrand is exactly cancelled by the second term, leaving only a principal value integral, which is well defined. Therefore, the quantity $\partial G\left(\omega_{0}, E_{m}^{(0)} / \partial \omega_{0}\right.$ in Eq. (4T) should more accurately be replaced by the quantity $\partial \widetilde{G}\left(\omega_{0}, E_{m}^{(0)}\right) / \partial \omega_{0}$ of Eq. (C13), which is not divergent as $r \rightarrow r_{s 0, j}$. In fact, it is important that in Eq. (C13) the contribution from the resonant region be small compared to that from the region around $r=r_{g}$ (where the gap mode is localized). The reason for this is that we have developed a perturbation theory in which the contribution from the integral near the resonant region is assumed to be small.

Incidentally, we note that by expressing the discontinuity of $E_{m}$ about $r_{s}$ in terms of $\Delta^{+}$ and $\Delta^{-}$with Eqs. (C6) and ( $\mathrm{C} 7$ ), we obtain the identity

$$
\begin{gathered}
\frac{\partial \tilde{G}\left(\omega_{0}, E_{m}^{(0)}\right)}{\partial \omega_{0}}=\lim _{\delta \omega \rightarrow 0}\left[\frac{\Delta^{+}\left(\omega_{0}+\delta \omega\right)-\Delta^{-}\left(\omega_{0}+\delta \omega\right)}{\delta \omega}\right] \frac{\left[C_{m}^{(0)}\left(r_{s 0}\right)\right]^{2}}{r_{s 0}^{3}\left[\frac{d}{d r}\left(\frac{\omega_{0}^{2}}{v_{A}^{2}}-k_{\| m}^{2}\right)\right]_{r_{s 0}}} \\
=\frac{\left[C_{m}^{(0)}\left(r_{s 0}\right)\right]^{2}}{r_{s 0}^{3}\left[\frac{d}{d r}\left(\frac{\omega_{0}^{2}}{v_{A}^{2}}-k_{\| m}^{2}\right)\right]_{r_{s 0}}} \frac{\partial}{\partial \omega_{0}}\left[\Delta^{+}\left(\omega_{0}\right)-\Delta^{-}\left(\omega_{0}\right)\right] .
\end{gathered}
$$

In practice, the principal value integral in the expression of Eq. (C13) for $\partial \widetilde{G}\left(\omega_{0}, E_{m}^{(0)}\right) / \partial \omega_{0}$ is difficult to integrate numerically because it contains relatively delicate cancellations. Also. the expression given by the right-hand side of Eq. (C14) is not convenient to evaluate. because of the singular nature of the differential equation near the resonance. A more convenient way to evaluate $\partial \tilde{G} / \partial \omega_{0}$ is given by another approach, which is based on our numerical method for searching for the eigenvalue as described in Sec. VI. In our method of solution, we solve the eigenmode equation given by Eq. (36) with the requirement that 
$E_{m}\left[r_{s}(\omega)+\eta\right]=E_{m}\left[r_{s}(\omega)-\eta\right]$, so that $E_{m}(a ; \omega)$ is nonzero, unless $\omega=\omega_{0}$ for which $E_{m}(a ; \omega)=0$. Here, the notation $E_{m}(a ; \omega)$ is introduced in order to emphasize the dependence of the solution on the (real) frequency shift. Ușing Eq. (C1) and taking into account the nonzero endpoint contribution at the edge, one can straightforwardly show that

$$
\frac{\partial \tilde{G}\left(\omega_{0}, E_{m}^{(0)}\right)}{\partial \omega_{0}}=-\lim _{\delta \omega_{r} \rightarrow 0} \frac{E_{m}\left(a ; \omega_{0}+\delta \omega_{r}\right) C_{m}\left(a ; \omega_{0}+\delta \omega_{r}\right)}{\delta \omega_{r}} .
$$

This result is extremely convenient, since our search routine for $\omega_{0}$ solves for $E_{m}(a ; \omega)$.

When toroidal mode coupling is present, Eqs. (C13) and (C15) have appropriate generalizations. It can be shown that

$$
\begin{aligned}
& \frac{\partial}{\partial \omega_{0}} \tilde{G}\left(\omega_{0} . E^{(0)}\right) \equiv \mathcal{P} \int_{0}^{a} \frac{d r}{r^{3}}\left\{\left(\sum_{m, n s, t} \frac{C_{s}^{(0)}(r) D^{s m}\left(\omega_{0}, r\right) \frac{\partial}{\partial \omega_{0}} D_{m n}\left(\omega_{0}, r\right) D^{n t}\left(\omega_{0}, r\right) C_{t}^{(0)}(r)}{\left\|D\left(\omega_{0}, r\right)\right\|^{2}}\right)+\cdots\right. \\
&\left.-\sum_{n, m, j} \frac{C_{n}^{(0)}\left(r_{s j}\right) D^{n m}\left(\omega_{0}, r_{s j}\right) C_{m}^{(0)}\left(r_{s j}\right) \frac{\partial}{\partial \omega_{0}}\left\|D\left(\omega_{0}, r_{s j}\right)\right\|}{\left[\left(r-r_{s j}\right) \frac{\partial}{\partial r}\left\|D\left(\omega_{0}, r_{s j}\right)\right\|\right]^{2}}\right\} \\
&-\sum_{n, m, j} \frac{a}{r_{s j}\left(a-r_{s j}\right)} C_{n}^{(0)}\left(r_{s j}\right) D^{n m}\left(\omega_{0}, r_{s j}\right) C_{m}^{(0)}\left(r_{s i}\right) \frac{\partial}{\partial \omega_{0}}\left\|D\left(\omega_{0}, r_{s j}\right)\right\| \\
&=-\sum_{m} \frac{\partial E_{m}(a: \omega)}{\partial \omega} C_{m}^{(0)}(a) .
\end{aligned}
$$

We also note that the quadratic singularity cancels at $r=r_{s}$ for, since $\left\|D\left(\omega_{0}, r_{s j}\right)\right\|=0$. we have

$$
\sum_{m, n} D^{s m}\left(\omega_{0}, r_{s j}\right) \frac{\partial}{\partial \omega_{0}} D_{m n}\left(\omega_{0}, r_{s j}\right) D^{n t}\left(\omega_{0}, r_{s j}\right)=D^{s t}\left(\omega_{0}, r_{s j}\right) \frac{\partial}{\partial \omega_{0}}\left\|D\left(\omega_{0} . r_{s j}\right)\right\| .
$$

For the three-mode coupling problem, we represented $E_{m}$ and $C_{m}$ by Eq. (89). The $e_{i}$ were so chosen that $E_{1}(a)=E_{3}(a)=0$, and $E_{2}(a) \equiv\|E(\omega)\|$, with $\| E(\omega \|$ given by Eq. (92). Thus. Eq. (C16) becomes

$$
\frac{\partial}{\partial \omega_{0}} \tilde{G}\left(\omega_{0}, E^{(0)}\right)=-\frac{\partial\|E(\omega)\|}{\partial \omega} C_{2}^{(0)}(a)
$$


and $\frac{\partial}{\partial \omega}\|E(\omega)\|$ is readily determined as a numerical difference.

Finally, we note that, although the amended form of $\partial G\left(\omega_{0}, E_{m}^{(0)}\right) / \partial \omega_{0}$ as given by $\partial \widetilde{G}\left(\omega_{0}, E_{m}^{(0)}\right) / \partial \omega_{0}$ either in Eq. (C13) or in Eq. (C16) is non-divergent, it is no longer positive definite. However, we note from Eqs. (15) and (16) in the text that the imaginary part of the energy functional $Q(\omega, \delta \Phi)$ is positive definite for $\omega$ in the upper half-plane, as long as $\delta W>0$. For an unstable root to exist, $G$ must vanish in the upper half $\omega$-plane; however, since $G=\omega Q$ and $\operatorname{Im} Q>0$ in the upper half plane, there are no unstable roots. Therefore, the conclusion that continuum resonance leads to damping when the system is MHD stable must hold. 


\section{Appendix D: Alternate Method for Estimating the Continuum Damping Rate}

The method described in the text for estimating the damping rate assumes that the eigenfunction is approximately real. This assumption can fail particularly when there are multiple points along the real axis where an Alfvén continuum resonance occurs for real $\omega$. Then, at each such point, a complex discontinuity in $E_{m}$ is induced, and it can arise that the accumulated imaginary part of the eigenfunction may not be ignorable between singular points. To deal with this possibility, we now suggest an alternate method for estimating the damping rate.

If $\omega$ is real, we can integrate Eq. (35), ignoring $L_{k}(\omega)$, with the assumption $E_{m}(r)$ and $C_{m}(r)$ are complex. At the origin we take regular boundary conditions as given by Eq. (10). with $E_{m}^{(i)}(r)$ and $C_{m}^{(i)}(r)$ real. We then integrate to just before the first singular point $r=r_{s 1}$. where $\left\|D\left(\omega, r_{s 1}\right)\right\|=0$. We now use the causality condition given by Eq. (62) to connect the amplitudes at $r_{s 1}+\eta$ to those at $r_{s 1}-\eta$ :

$$
\begin{aligned}
& C_{m}^{(i)}\left(r_{s 1}+\eta\right)=C_{m}^{(i)}\left(r_{s 1}-\eta\right) \\
& E_{m}^{(i)}\left(r_{s 1}+\eta\right)=E_{m}^{(i)}\left(r_{s 1}-\eta\right)
\end{aligned}
$$

$$
\frac{-i \pi}{r_{s 1}^{3}} \sum_{n} \frac{\operatorname{sgn}\left(\frac{\partial\|D\|}{\partial \omega}\right) D^{m n}\left(\omega, r_{s 1}\right) C_{n}^{(i)}\left(r_{s 1}\right)}{\left|\frac{\partial}{\partial r_{s 1}}\left\|D\left(\omega, r_{s 1}\right)\right\|\right|} .
$$

We can thus continue the integration, with $E$ and $C$ now complex. Note that subsequent integration of the differential equation induces an imaginary part in $C$ from the imaginary discontinuity that is given in Eq. (D2). At each successive singularity, a discontinuity is induced as given by Eqs. (D1) and (D2) (with $r_{s j}$ replacing $r_{s 1}$ ), but now with $C_{m}^{(i)}\left(r_{s j}\right)$ and $E_{m}^{(i)}\left(r_{s j}-\eta\right)$ being complex. We integrate these equations to the boundary $r=a$ where we attempt to satisfy the boundary condition $E_{m}(a)=0$. An appropriate solution should have 
the form of Eq. (91) (but now with $e_{i}(\omega)$ complex), which leads to the dispersion relation given by Eq. (92)

$$
\|E(\omega)\|=0
$$

Now in general $\|E(\omega)\|$ is not real for real $\omega$, and it will not have real eigenvalues. However, $\|E(\omega)\|$ is an analytic function of $\omega$, and its zeros are in the complex plane where $\operatorname{Im} \omega \equiv \gamma<0$. If $\gamma$ is sufficiently small, we can obtain an estimate of $\gamma$ as follows. Let

$$
\|E(\omega)\|=\varepsilon_{R}(\omega)+i \varepsilon_{I}(\omega)
$$

where $\varepsilon_{R}(\omega)$ and $\varepsilon_{I}(\omega)$ are both real functions of $\omega$. Let $\omega_{0}$ be defined as the real frequency for which

$$
\varepsilon_{R}\left(\omega_{0}\right)=0
$$

Then, if we take $\omega=\omega_{0}+i \gamma$, by expanding in a power series and neglecting higher order terms, we obtain

$$
\gamma=-\frac{\varepsilon_{I}\left(\omega_{0}\right)}{\partial \varepsilon_{R}\left(\omega_{0}\right) / \partial \omega_{0}}
$$

The conditions for neglecting higher-order terms of the power series are

$$
\frac{\left|\varepsilon_{I}\left(\omega_{0}\right) \frac{\partial^{2} \varepsilon_{R}\left(\omega_{0}\right)}{\partial \omega_{0}}\right|}{\left(\frac{\partial \varepsilon_{R}\left(\omega_{0}\right)}{\partial \omega_{0}}\right)^{2}} \cdot\left|\frac{\frac{\partial \varepsilon_{I}\left(\omega_{0}\right)}{\partial \omega_{0}}}{\frac{\partial \varepsilon_{R}\left(\omega_{0}\right)}{\partial \omega_{0}}}\right|<1 .
$$

In Fig. 13 we compare the damping rates computed using both the method of the text (solid curve) and the method described in this appendix, for the density profile given in Eq. (98) and for the parameters stated thereafter. There is very good agreement between the predictions of the two methods except in the vicinity of $q(a) \approx 3$. In this case the alternate method generally gives a larger damping rate and is more credible.

We also tabulate in Table II the parameter

$$
\delta \equiv\left|\frac{\varepsilon_{I}\left(\omega_{0}\right) \frac{\partial^{2} \varepsilon_{R}\left(\omega_{0}\right)}{\partial \omega^{2}}}{\frac{\partial \varepsilon_{R}\left(\omega_{0}\right)}{\partial \omega}}\right|
$$


as a function of $q(a)$. According to Eq. (D7), This quantity needs to be much less than unity in order to justify the damping rate estimale. We see there are regions in which the validity of our estimates appears to be marginal. In these regions the damping rate seems to have a larger value than in regions where the theory is valid, and hence destabilization from alpha particles is less of a concern. However, if the correct damping rate is desired in these regions, one should either develop a code that can integrate in the complex $r$-plane along a contour on which $\operatorname{Im} \frac{1}{\omega}\|D(\omega, r)\|>0$, or use a code with additional physics included, such as resistivity. Such an extension of this problem is not investigated in the present work. 


\section{References}

1. C.Z. Cheng and M.S. Chance, Phys. Fluids 29, 3695 (1986).

2. G.Y. Fu, Ph.D Thesis, The University of Texas (1988); Institute for Fusion Studies Report, No. IFSR-325 (1988).

3. G.Y. Fu and J.W. Van Dam, Phys. Fluids B 1, 1949 (1989).

4. C.Z. Cheng, G.Y. Fu, and J.W. Van Dam, in Theory of Fusion Plasmas, ed. J. Vaclavik, F. Troyon, and E. Sindoni (Societa Italiana di Fisica/Editrice Compositori, Bologna. 1989), p. 259.

5. G.Y. Fu et al.. in Plasma Physics and Controlled Nuclear Fusion Research 1988 (International Atomic Energy Agency, Vienna, 1989), Vol. 2, p. 291.

6. J.W. Van Dam, G.Y. Fu, and C.Z. Cheng, Fusion Technology 18, 461 (1990).

i. C.Z. Cheng, Fusion Technology 18, 443 (1990).

8. C.Z. Cheng, Phys. Fluids B 2. 1427 (1990).

9. C.Z. Cheng, R.B. White, G.Y. Fu, L. Chen, D.J. Sigmar, C.T. Hsu, A. Beklemishev. H.L. Berk. B. Breizman, S.C. Guo, Z. Guo, D. Lindberg, and J.W. Van Dam. in Thirteenth International Conference on Plasma Physics and Controlled Nuclear Fusion Research (Washington, D.C., 1-6 October 1990), paper IAEA-CN-53/D-3-6, to be published.

10. D.J. Sigmar, C.T. Hsu, R.B. White, and C.Z. Cheng, MIT Report, No. PFC/JA-89-58 (1989).

11. H.L. Berk and B.N. Breizman, Phys. Fluids B 2, 2246 (1990). 
12. D.M. Meade et al., in Thirteenth International Conference on Plasma Physics and Controlled Nuclear Fusion Research (Washington, D.C., 1-6 October 1990), paper IAEACN-53/A-1-1, to be published.

13. W.W. Heidbrink, E.J. Strait, E. Doyle, G. Sager, and R. Snider, "An Investigation of Beam-Driven Alfven Instabilities in the DIII-D Tokamak," submitted to Nucl. Fusion.

14. K.L. Wong et al., Phys. Rev. Lett. 66, 1874 (1991).

15. D.E. Baldwin and D.W. Ignat, Phys. Fluids 12. 697 (1969).

16. W. Grossman and J. Tataronis, Z. Phys. 261, 203, 217 (1973).

17. J.A. Tataronis, J. Plasma Phys. 13, 87 (1975).

1S. J.M. Kappraff and J.A. Tataronis, J. Plasma Phys. 18, 209 (197T).

19. A. Hasegawa and L. Chen. Phys. Rev. Lett. 32. 454 (1974).

20. L. Chen and A. Hasegawa, Phys. Fluids 17, 1399 (1974).

21. H.L. Berk and T.B. Kaiser. Phys. Fluids 28, 345 (1985).

22. F. Zonca and L. Chen. Bull. Am. Phys. Soc. 35. 2069 (1990).

23. D.W. Ross, G.L. Chen. and S.M. Mahajan, Phys. Fluids 25. 652 (1982).

24. K. Appert, R. Gruber, F. Troyon. and J. Vaclavik, in Proceedings of the 3rd Joint Varenna-Grenoble International Symposium. Grenoble. 1982 (ECSC-EEC-EAEC Euratom. Brussels, 1982), Vol. 1, pp. 203-212 (1982).

25. K. Appert, R. Gruber, F. Troyon, and J. Vaclavik, Plasma Phys. 24, 1147 (1982).

26. S.M. Mahajan, D.W. Ross, and G.L. Chen, Phys. Fluids 26, 2195 (1983). 
27. Y.M. Li, S.M. Mahajan, and D.W. Ross, Phys. Fluids 30, 1466 (1987).

28. G.Y. Fu and J.W. Van Dam, Phys. Fluids B 1, 2404 (1989).

29. O.P. Pogutse and E.I. Yurchenko, Nucl. Fusion 18, 1629 (1978).

30. D.A. D'Ippolito and J.P. Goedbloed, Plasma Phys. 22, 1091 (1980).

31. C.E. Kieras and J.A. Tataronis, J. Plasma Phys. 28, 395 (1982).

32. S. Riyopoulos and S. Mahajan, Phys. Fluid 29, 731 (1986).

33. C.Z. Cheng, L. Chen, and M.S. Chance, Ann. Phys. (NY) 161, 21 (1984).

34. G.Y. Fu and C.Z. Cheng, Phys. Fluids B 2, 985 (1990).

35. K. Hain and R. Lüst. Z. Naturforsch. 13a, 936 (1958).

36. J.P. Goedbloed and H.J.L. Hagebeuk, Phys. Fluids 15, 1090 (1972).

37. K. Appert. R. Gruber, and J. Vaclavik, Phys. Fluids 17, 1471 (1974).

38. M... Rosenbluth and P.H. Rutherford, Phys. Rev. Lett. 34. 1428 (1975).

39. K.T. Tsang, D.J. Sigmar, and J.C. Whitson, Phys. Fluids 24. 1508 (1981).

40. B. Coppi, A. Ferreira, and J.J. Ramos, Nucl. Fusion 19, 715 (1979); Phys. Rer. Lett. $44,990(1980)$.

41. J.D. Meiss and R.D. Hazeltine, The University of Texas, Institute for Fusion Studies Report, IFSR-399 (1989).

42. V.D. Shafranov, in Reviews of Plasma Physics, ed. M.A. Leontovich (Consultants Bureau, New York, 1966), Vol. 2, p. 103.

43. J.G. Cordey, R.J. Goldston, and D.R. Mikkelsen, Nucl. Fusion 21, 581 (1981). 
44. S. Glasstone and R.H. Lovberg, Controlled Thermonuclear Reactions (Robert E. Krieger, Huntington, N.Y., 1975). 


\section{Table I}

Nominal Parameters of Experimental Machines

$\begin{array}{cccc} & \text { TFTR } & \text { CIT } & \text { ITER } \\ R(\mathrm{~m}) & 2.5 & 2.1 & 6.0 \\ a(\mathrm{~m}) & 0.8 & 0.65 & 2.15 \\ B(\mathrm{~T}) & 5 & 11 & 4.85 \\ n_{e}\left(\mathrm{~m}^{-3}\right) & 1 \times 10^{20} & 4 \times 10^{20} & 1 \times 10^{20} \\ T(\mathrm{keV}) & 10 & 10 & 10 \\ \beta & 0.032 & 0.026 & 0.034 \\ a / R & 0.32 & 0.31 & 0.35 \\ \beta_{\alpha} & 1.9 \times 10^{-3} & 1.5 \times 10^{-3} & 2.0 \times 10^{-3} \\ \rho_{\alpha} / a & 0.094 & 0.31 & 0.036 \\ x_{A}=\frac{v_{A}}{v_{\alpha 0}} & 0.53 & 0.58 & 0.51 \\ v_{A} / 3 R_{0}(\mathrm{kHz}) & 9.2 \times 10^{2} & 1.2 \times 10^{3} & 3.7 \times 10^{2}\end{array}$




\section{Figure Captions}

1. (a) Formation of Gap Due to Toroidicity. The dotted curve represents the Alfvén resonance frequency as a function of radius for the $n=1, m=1$ and $n=1, m=2$ modes, which intersect where $1-1 / q(r)=-1+2 / q(r)$. The solid curve represents the frequency spectrum with the effect of toroidicity included. With toroidicity there is no intersection; instead a gap arises between the $\omega^{+}$and $\omega^{-}$branches. Away from the gap region, the predominant $m$-number of the resonance can be identified. Here $\Omega=\omega R_{0} / v_{A}(0), a / R_{0}=0.25$, and $q(r)=1+r^{2} / a^{2}$.

(b) Eigenmode Structure of the TAE Mode. The structure of the global mode amplitude as a function of radius, for the parameters given in Fig. 1(a). The eigenfrequency is $\Omega=0.31$.

2. Gap Structure with Satellite Resonance. The density decrease at the edge of the plasma causes an additional resonance to arise near the plasma edge. The solid line is the global TAE eigenfrequency. The resonance at the edge can cause intrinsic damping. as described in this paper. In this case $q(r)=1+1.3 r^{2} / a^{2}$.

3. Multiple Gap Structure. Two gaps can line up, which can cause multiple mode structure. For this case $q=1+2 r^{2} / a^{2}$. The solid curves indicate the global eigenfrequencies that were found.

4. Discontinuity Parameter. Plot of the discontinuity parameter $\sigma$ as a function of edge $q(a)$ for flat density profile, with $\Omega=1 / 3$.

5. Comparison of Analytic to Numerical Eigenfrequencies. The short solid lines indicate the predictions of analytic theory for $\left(\Omega-\Omega_{0}\right) R_{0} / a$. The numerical curves, with the indicated edge- $q$ values, approach the analytic results as $a / R_{\mathrm{J}} \rightarrow 0$.

6. Flux Factor Ratio $\left(C\left(r_{s}\right) / C\left(r_{g}\right)\right)^{2}$. The ratio of $\left[C_{2}\left(r_{s}\right) / C_{2}\left(r_{g}\right)\right]^{2}$ is plotted as a function 
of edge $q$ for the density profile of Eq. (78). The dashed curves are numerical results for different values of the aspect ratio, whereas the solid curve is the prediction of analytic theory for $a / R \rightarrow 0$.

7. Eigenfrequency and Damping Rate. Eigenfrequency, $\Omega$, and damping rate, $\gamma$, as a function of edge $q$, with $a / R_{0}=0.25$, for three density profiles: (a) the density profile of Eq. (78); (b) the density profile that decreases slowly in the center and rapidly at edge, as described in the text prior to Eq. (98); and (c) the density profile given in Eq. (98).

8. "Smooth" Density Profile. The normalized density profile $g(r)$ of Eq. (98), with $\Delta_{1}=$ 0.2 and $\Delta_{2}=0.05$.

9. Double Gap Alignment. Plot of the Alfvén resonance frequency for the density profile of Eq. (98), the parameters of Fig. $7(\mathrm{c})$, and $q(a)=3.165$. The gaps for $m=1.2$ and $m=2,3$ align, and two global TAE modes are found at $\Omega=0.389$ and $\Omega=0.313$. Here $\Delta_{1}=0.2$ and $\Delta_{2}=0.05$.

10. Eigenfunctions for the Double Gap Alignment of Fig. 9. (a) The amplitudes $E_{m}$ and $C_{m}$ for $\Omega=0.389$; (b) $E_{m}$ and $C_{m}$ for $\Omega=0.313$. Note that the $C_{m}(r)$ plotted in these graphs is actually the $C_{m}(r)$ of the paper multiplied by $R_{0}^{2}$.

11. Sensitivity of Alpha Particle Growth Rate to Scale Length. The alpha particle growth as a function of $\omega_{*_{\alpha}} / \omega$ for different temperatures. For typical reactor parameters (described in the text) $\omega_{*_{\alpha}} / \omega=\lambda / \ell_{\alpha}$, where $\ell_{\alpha}$ is the alpha particle scale length at the gap position $r=r_{g}$, and the value of $\lambda$ is device-dependent, as given in Eq. (99). The solid curve is for constant density, whereas the dashed and dotted curves are for a constant plasma beta of $3 \%$. 
12. Plots of functions appearing in alpha particle drive to growth rate: (a) $3 x_{A} P_{\alpha}\left(x_{A}\right)$; (b) $10 x_{A} Q_{\alpha}\left(x_{A}\right)$.

13. Comparison of the damping rates calculated with the modified method of Appendix D (dashed curve) and with the method described in the text, (solid curve), for the parameters given in Fig. 7. The two results agree extremely well if only one resonant point is present, whereas there is significant deviation when more than one resonance point occurs. 


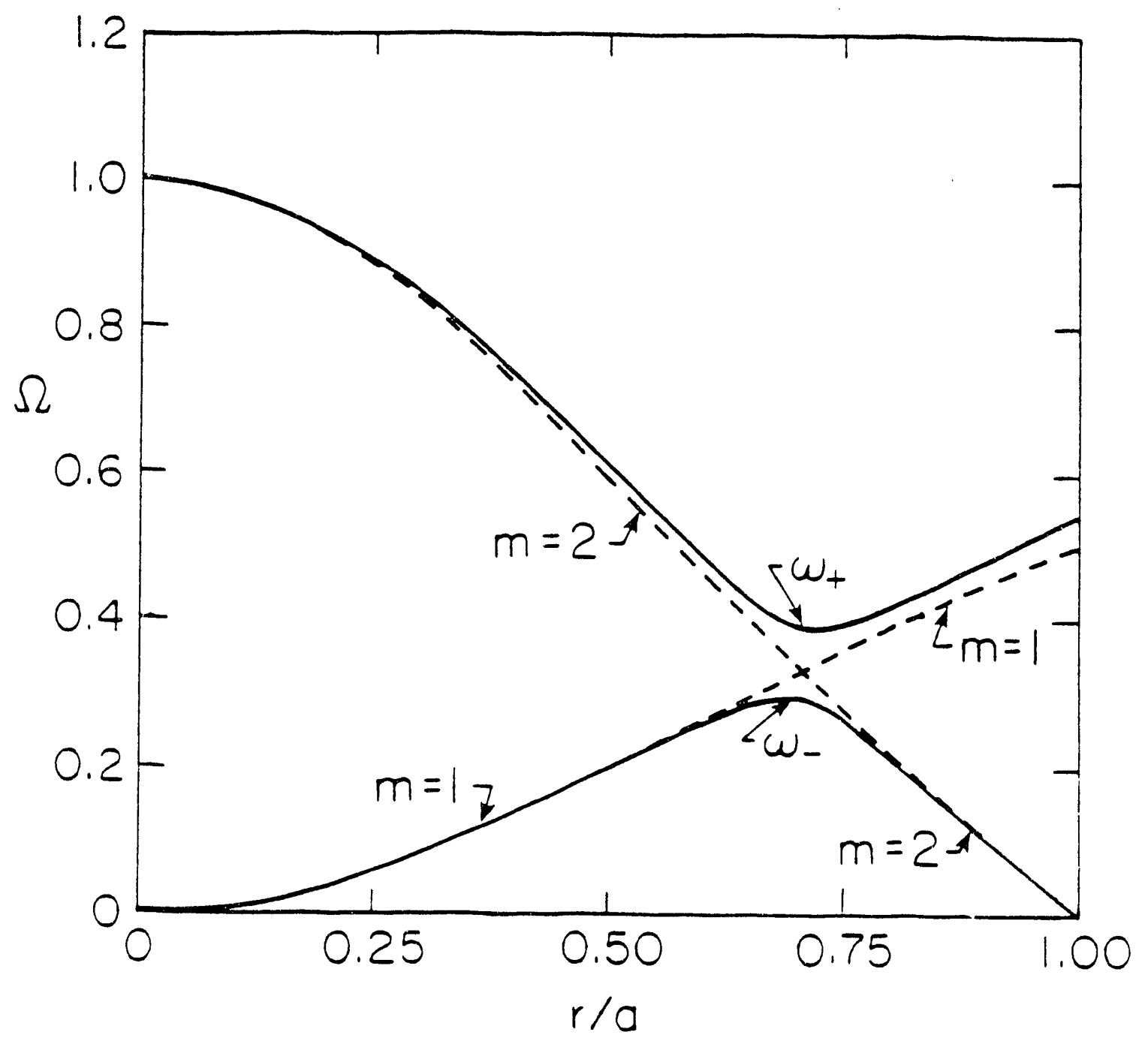

Fig. la 


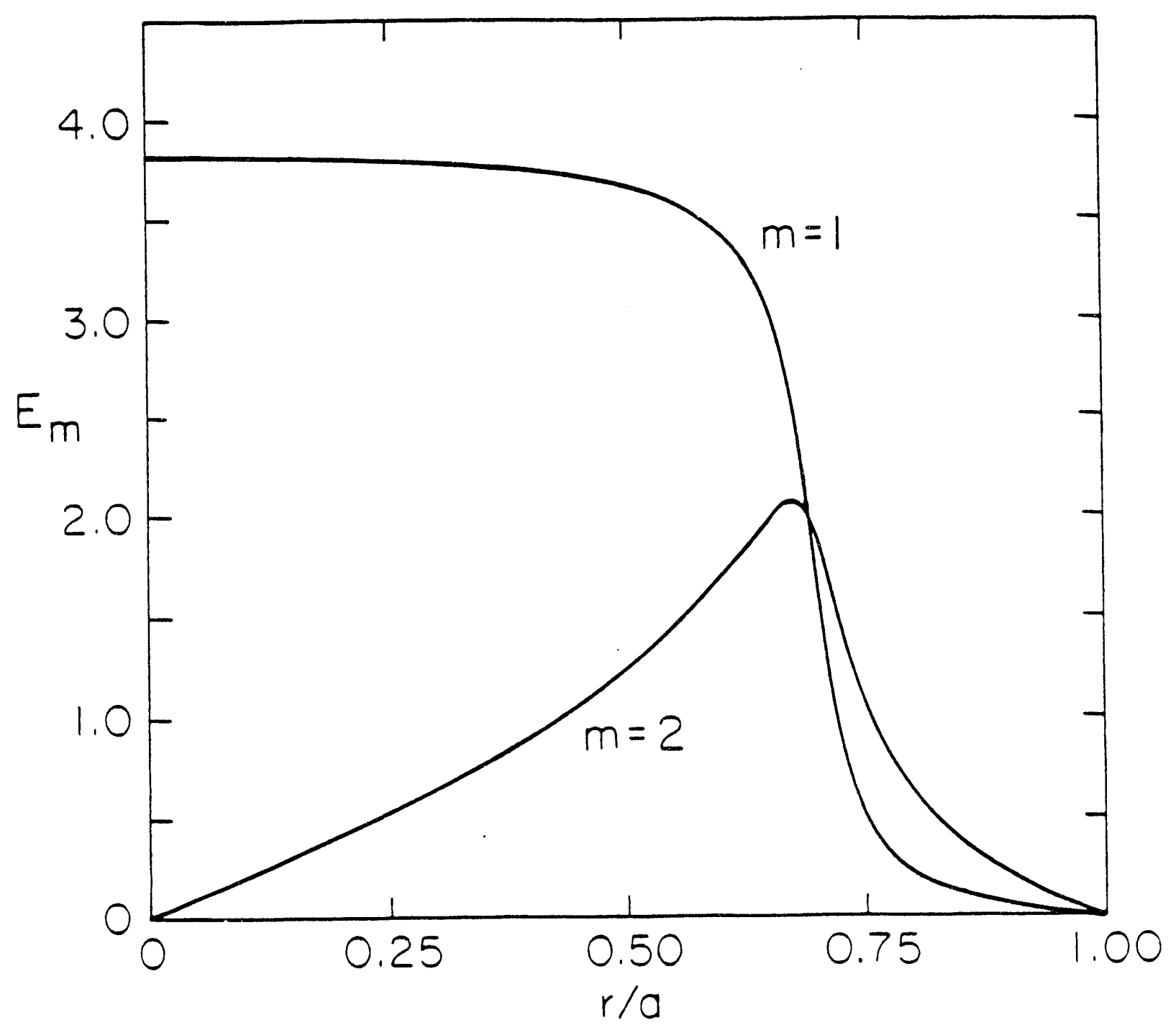

Fig. Ib 


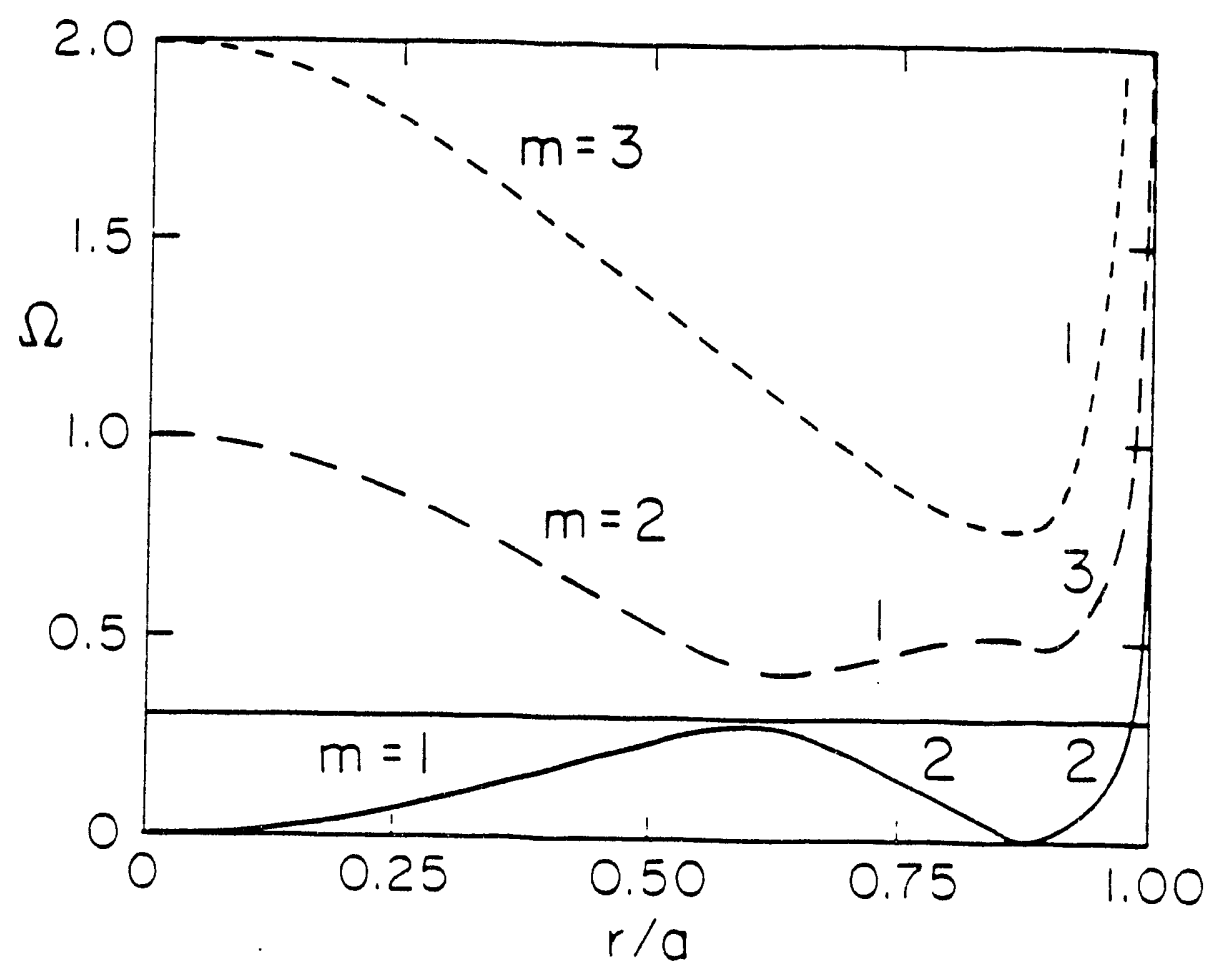

Fig. 2 


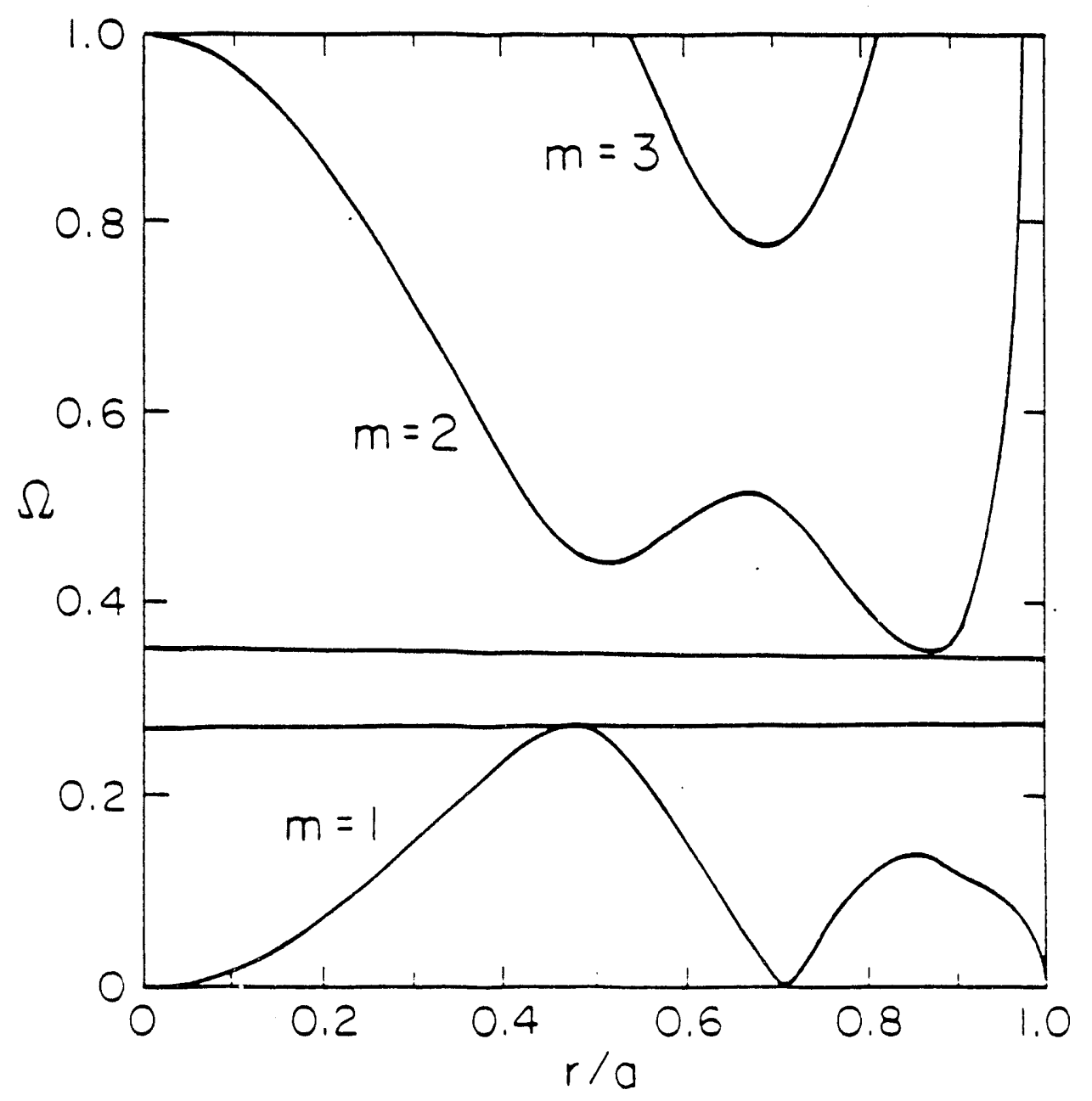

41 


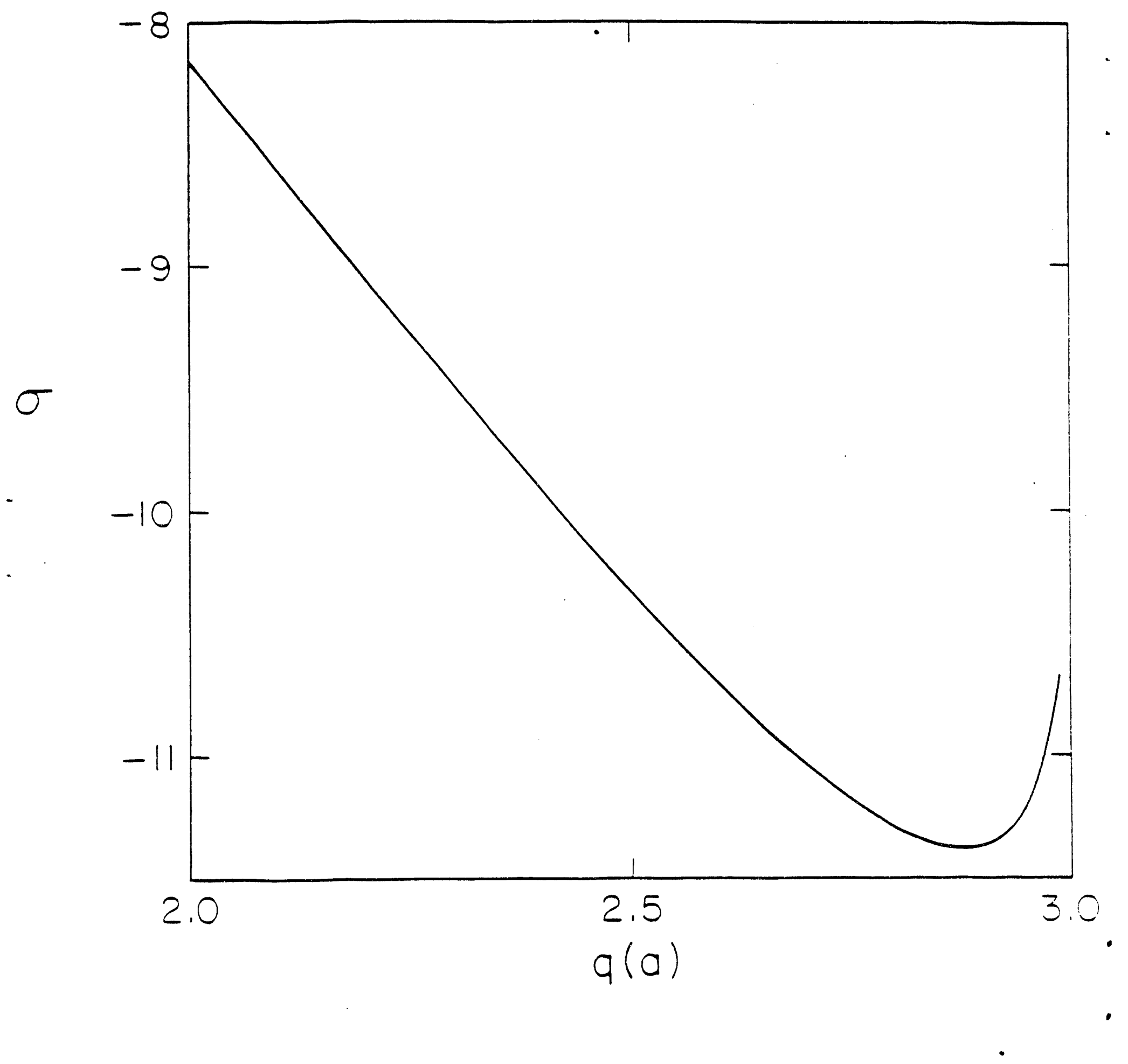

Fig. 4 


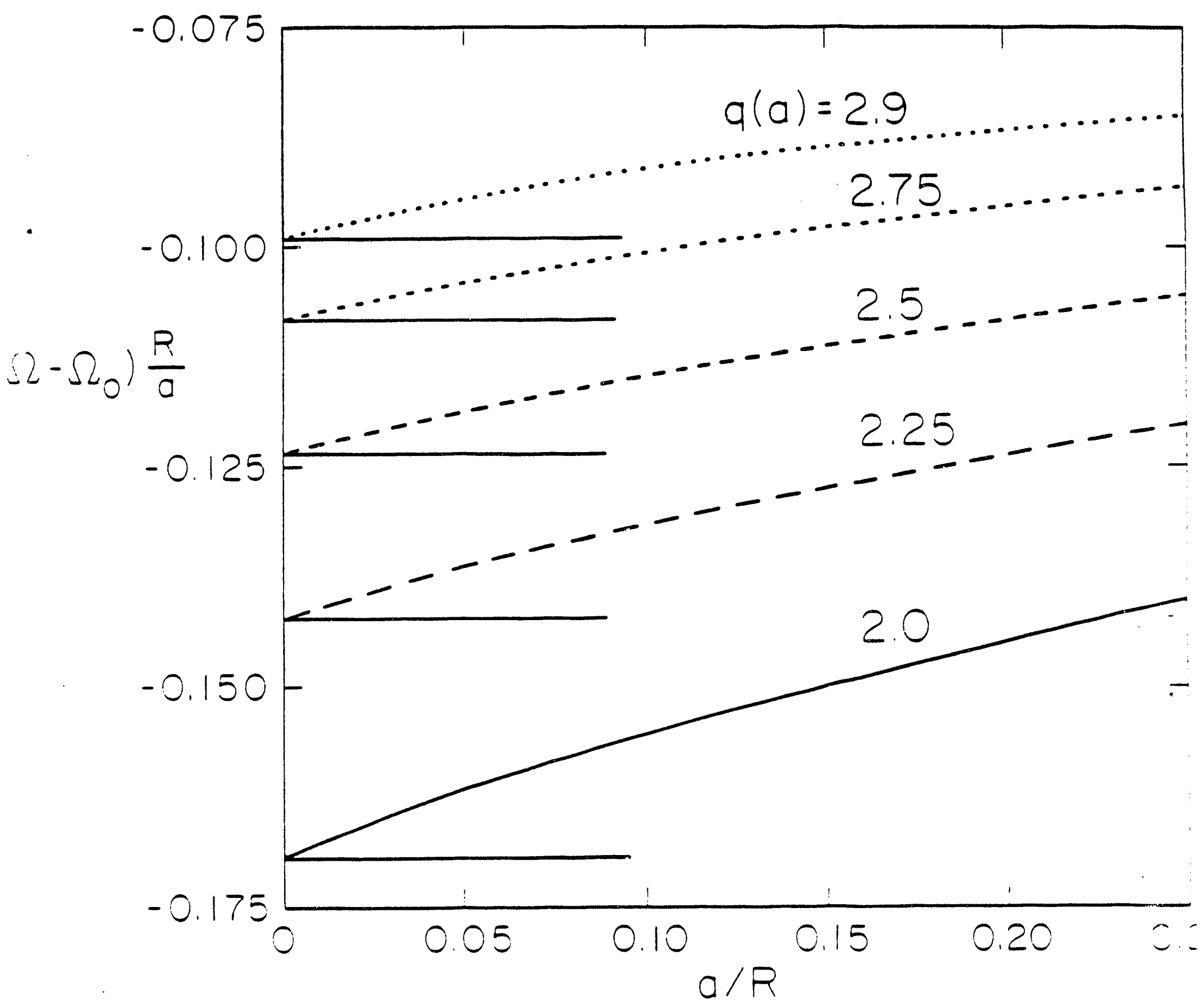




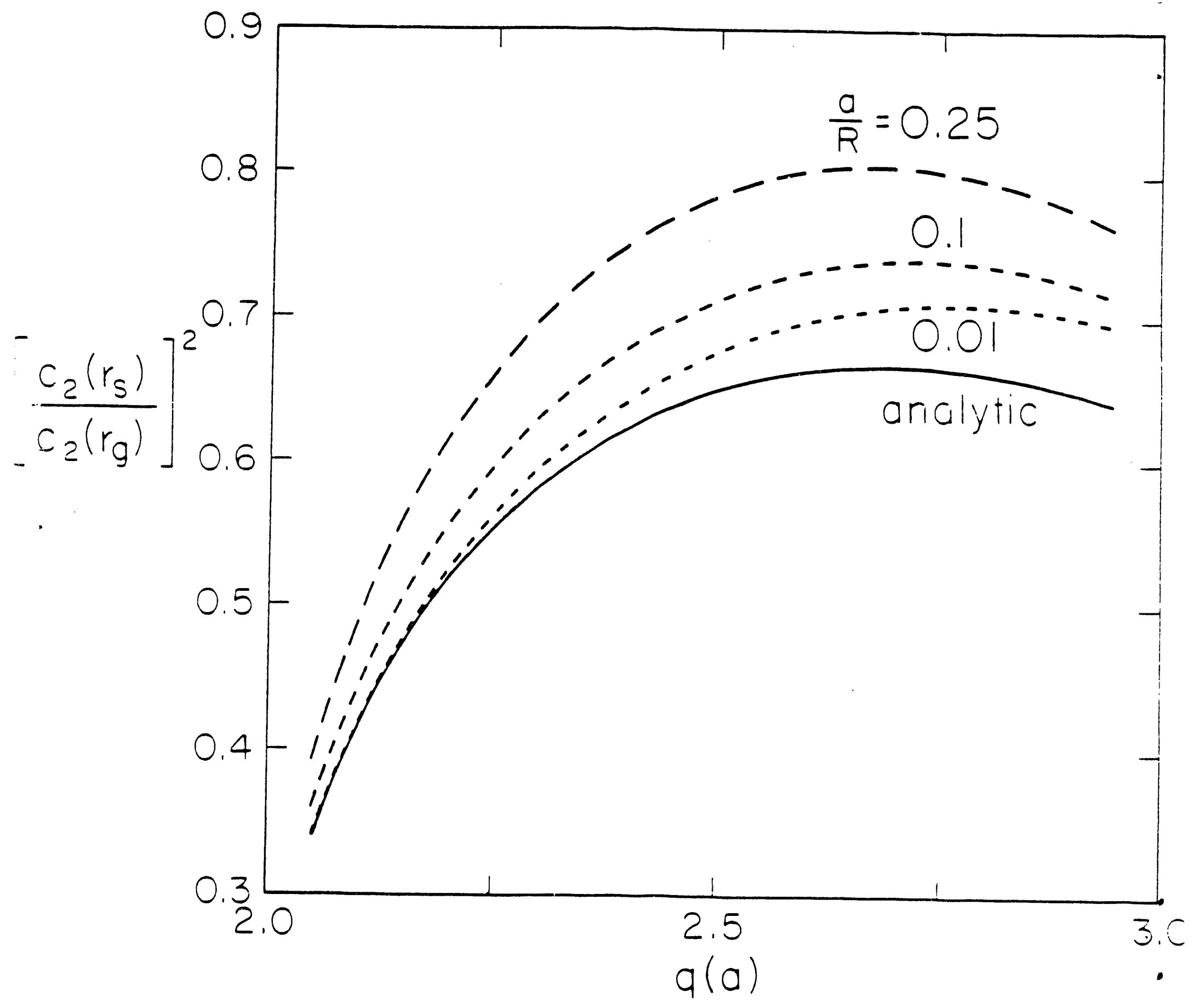




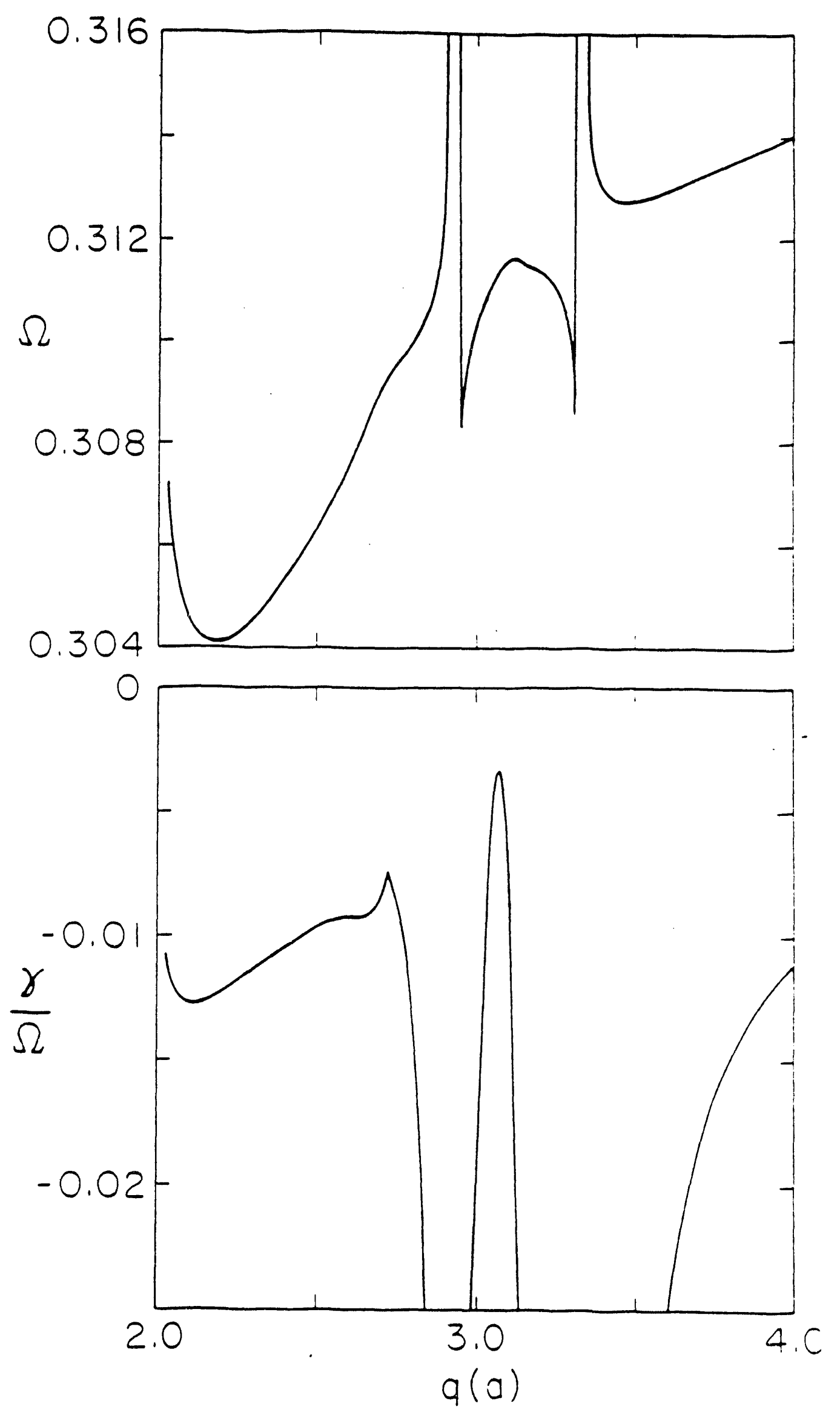

Fig. $7(a)$ 


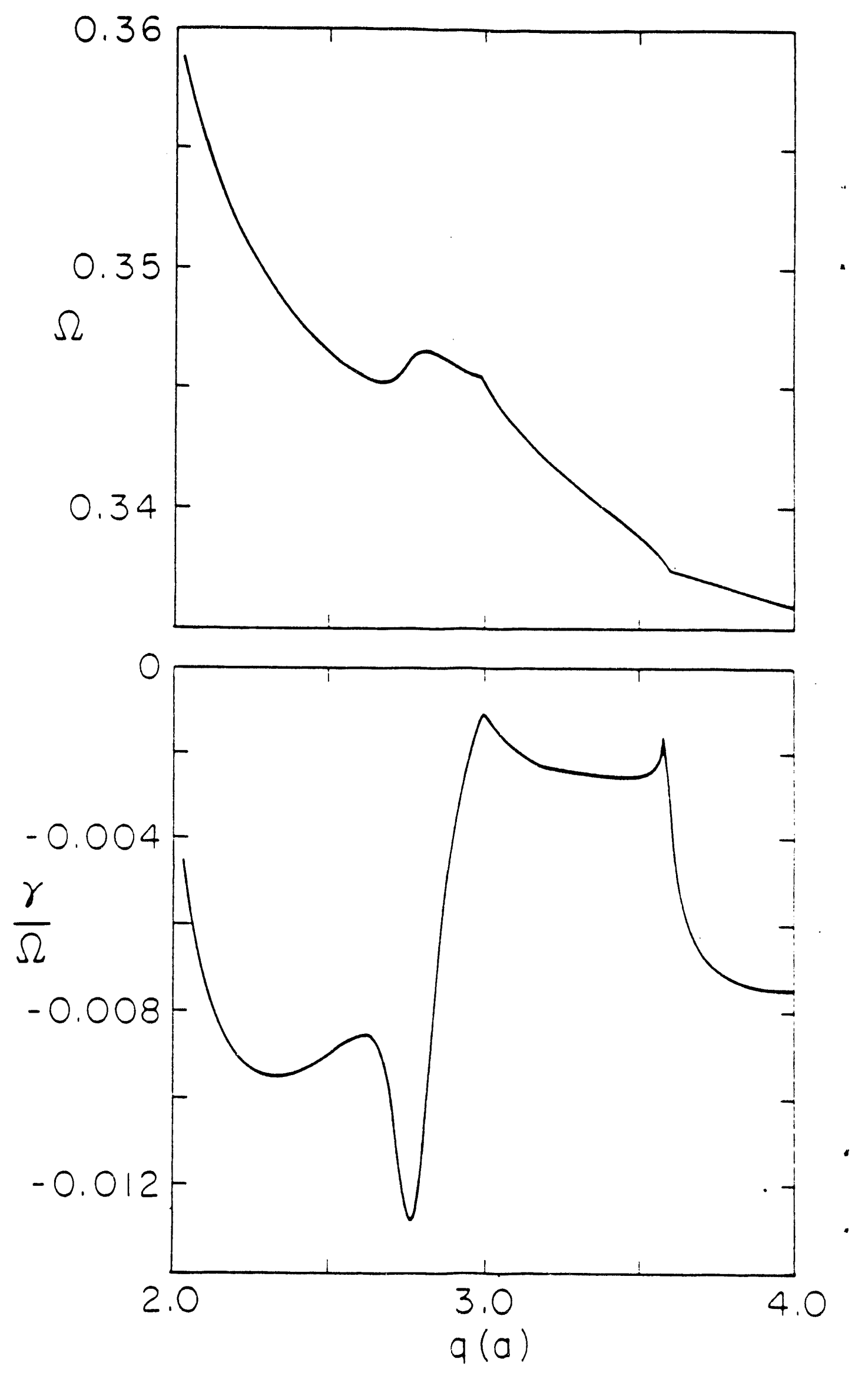

Fig. 7b 


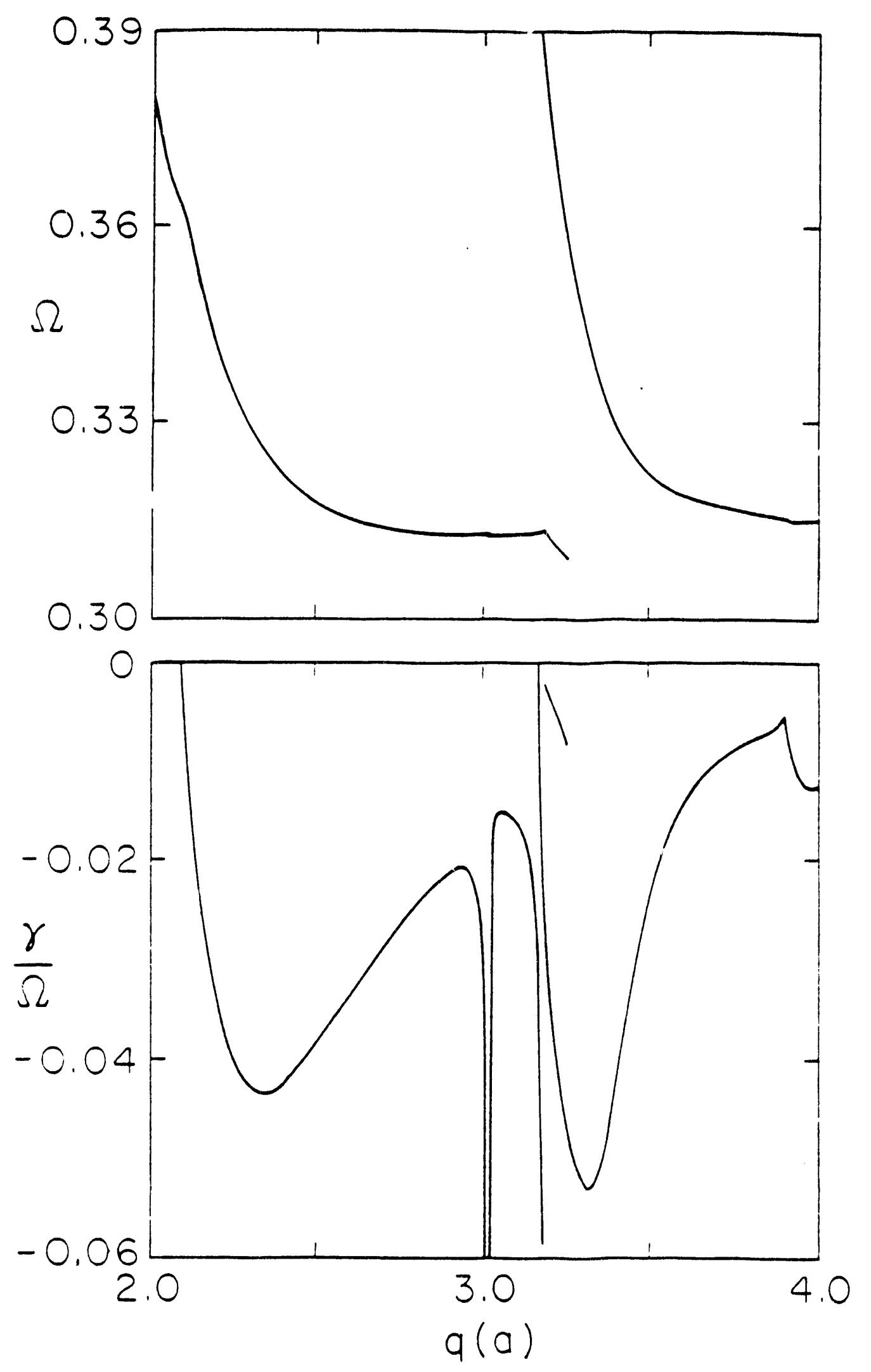

Fig. 7 (c) 


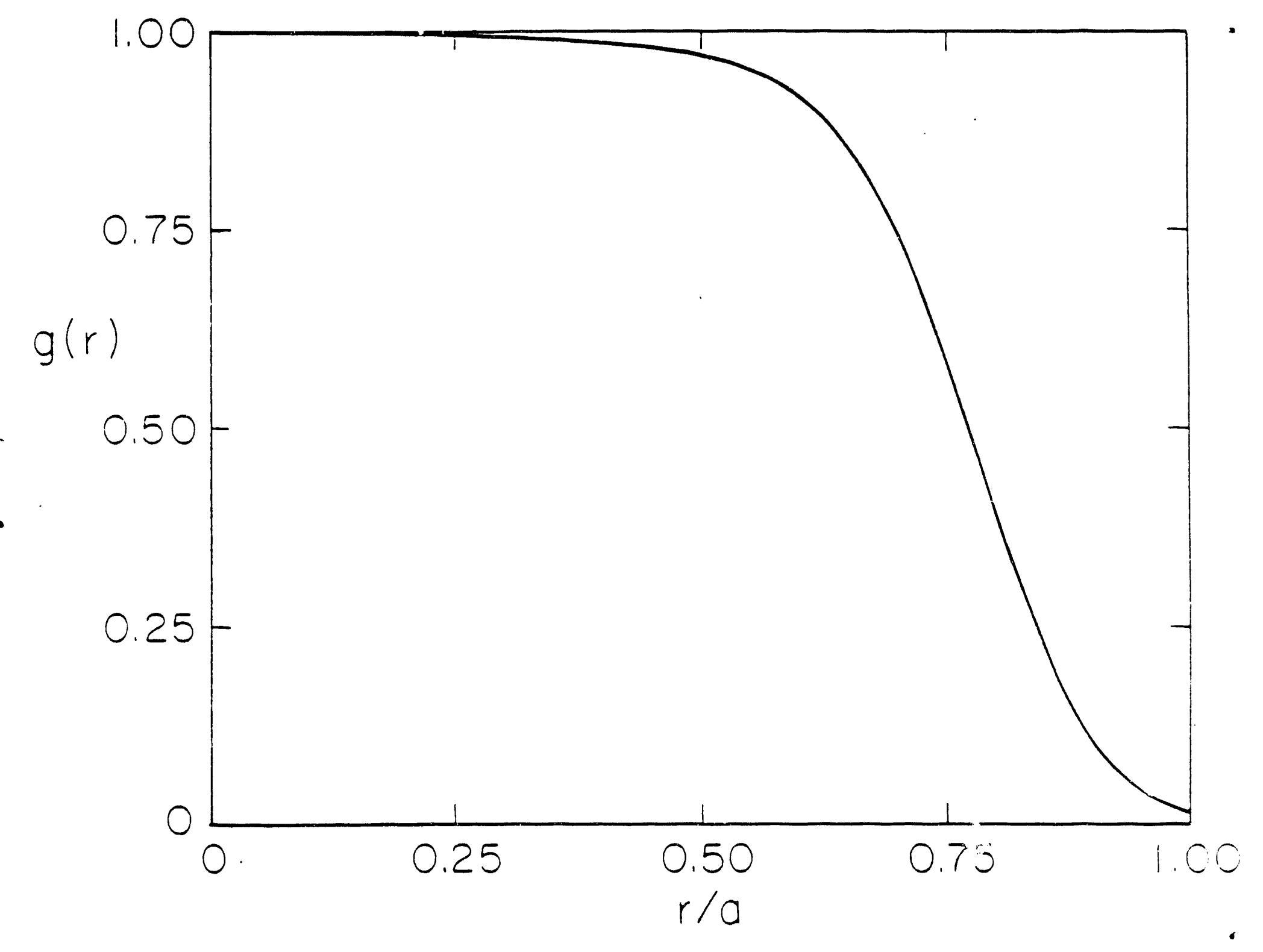

Fig. 8 


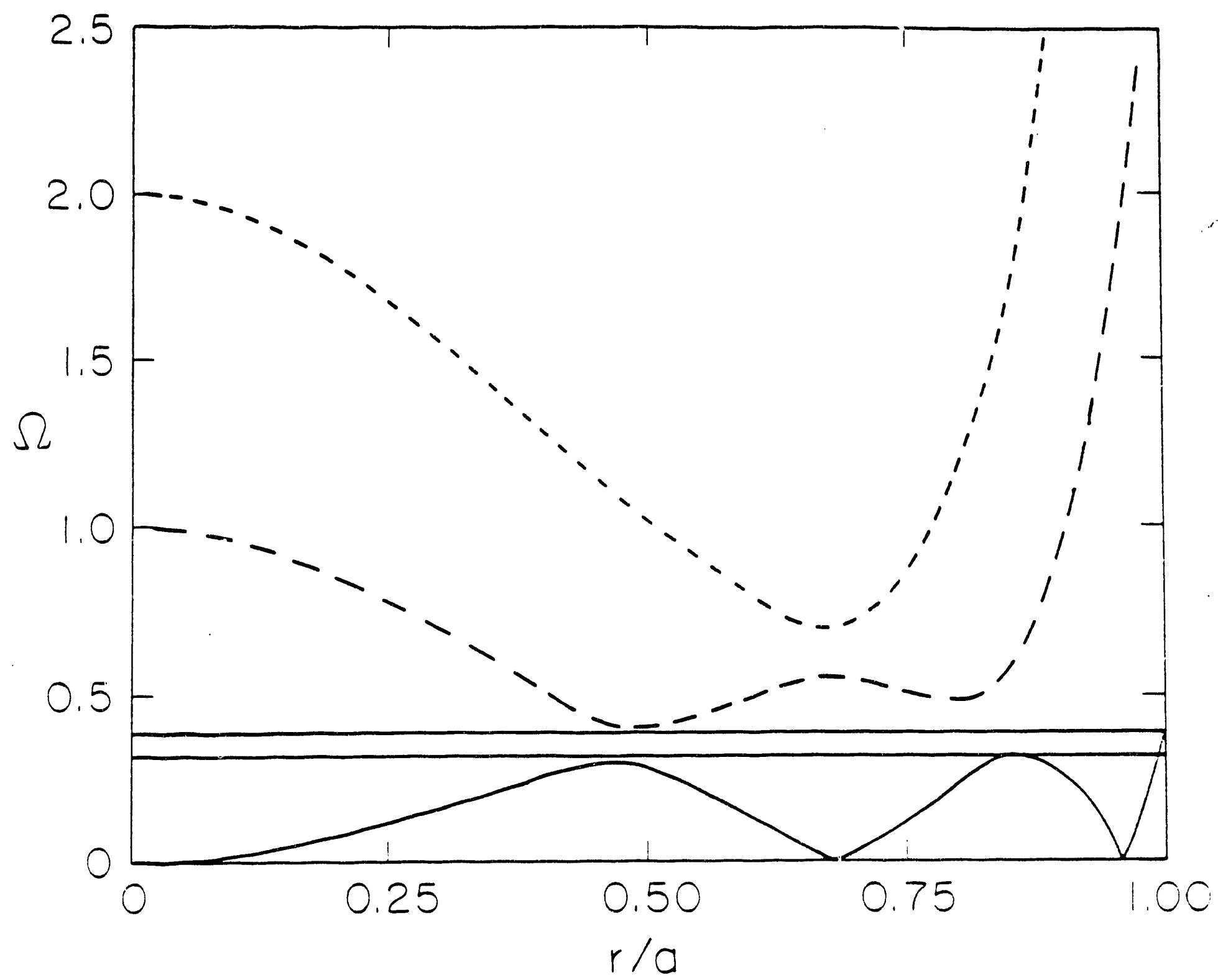

Fig. 9 

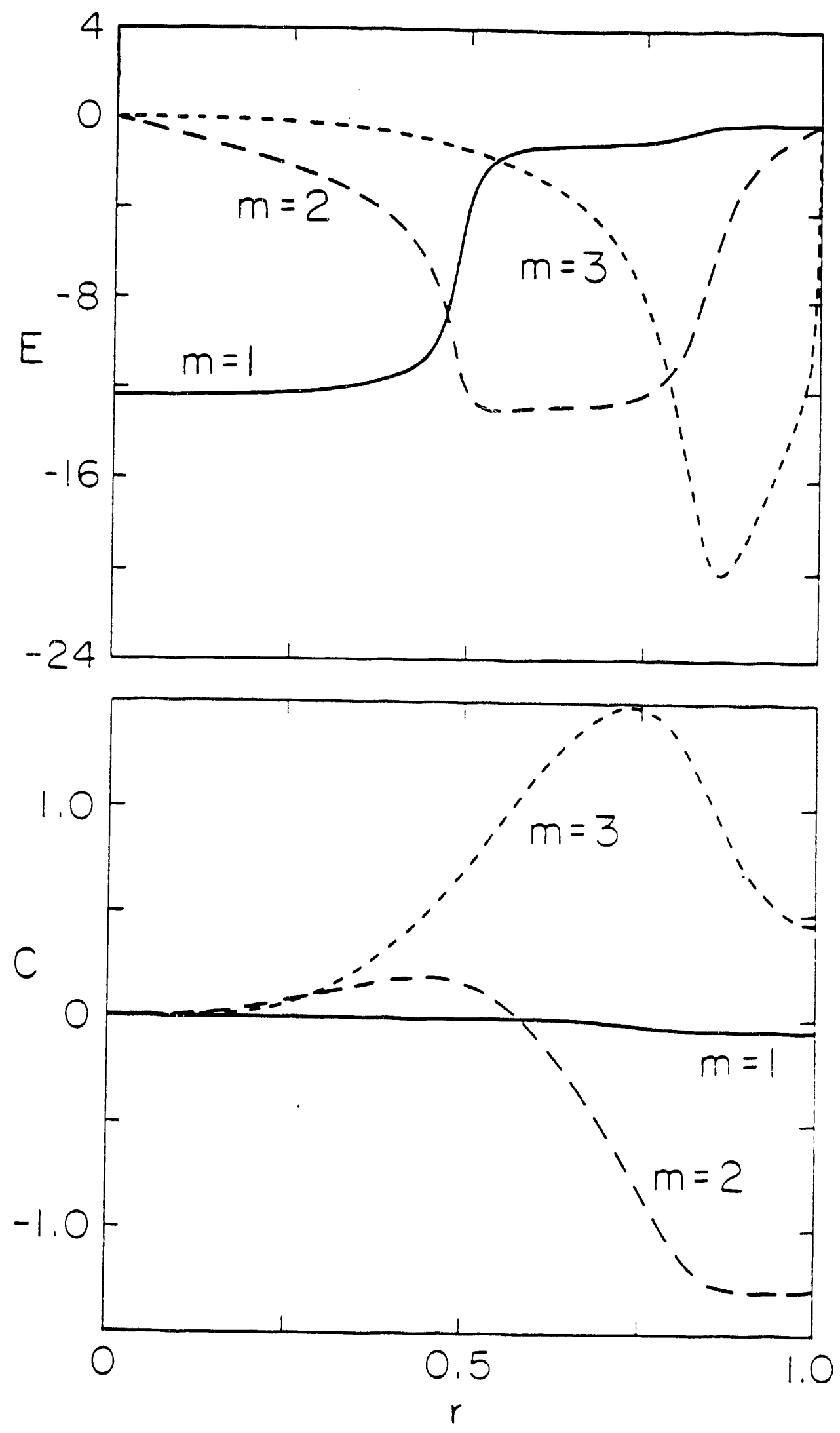

Fig. 10a 

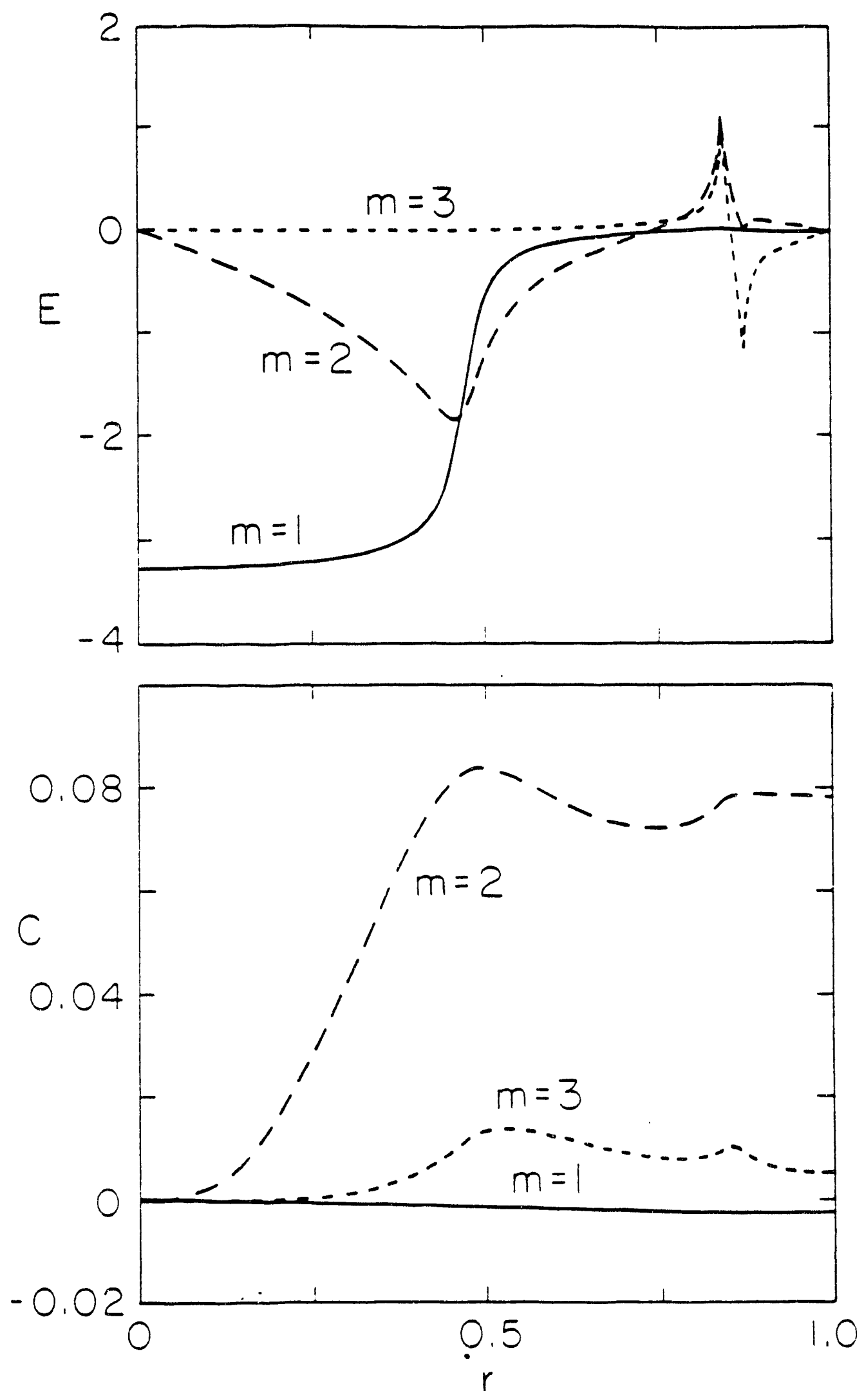

Fig. 10b 


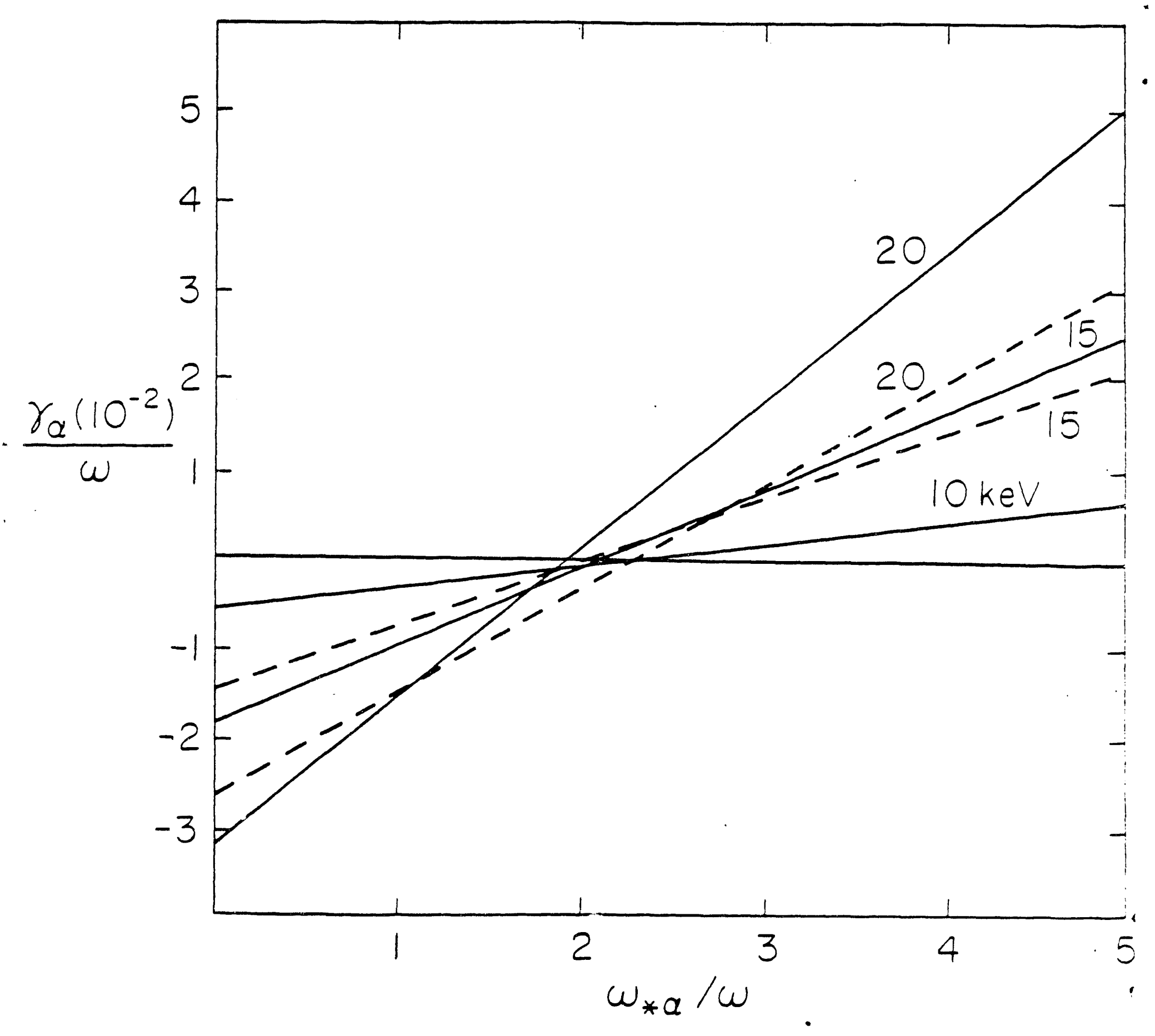

Fig. 11 


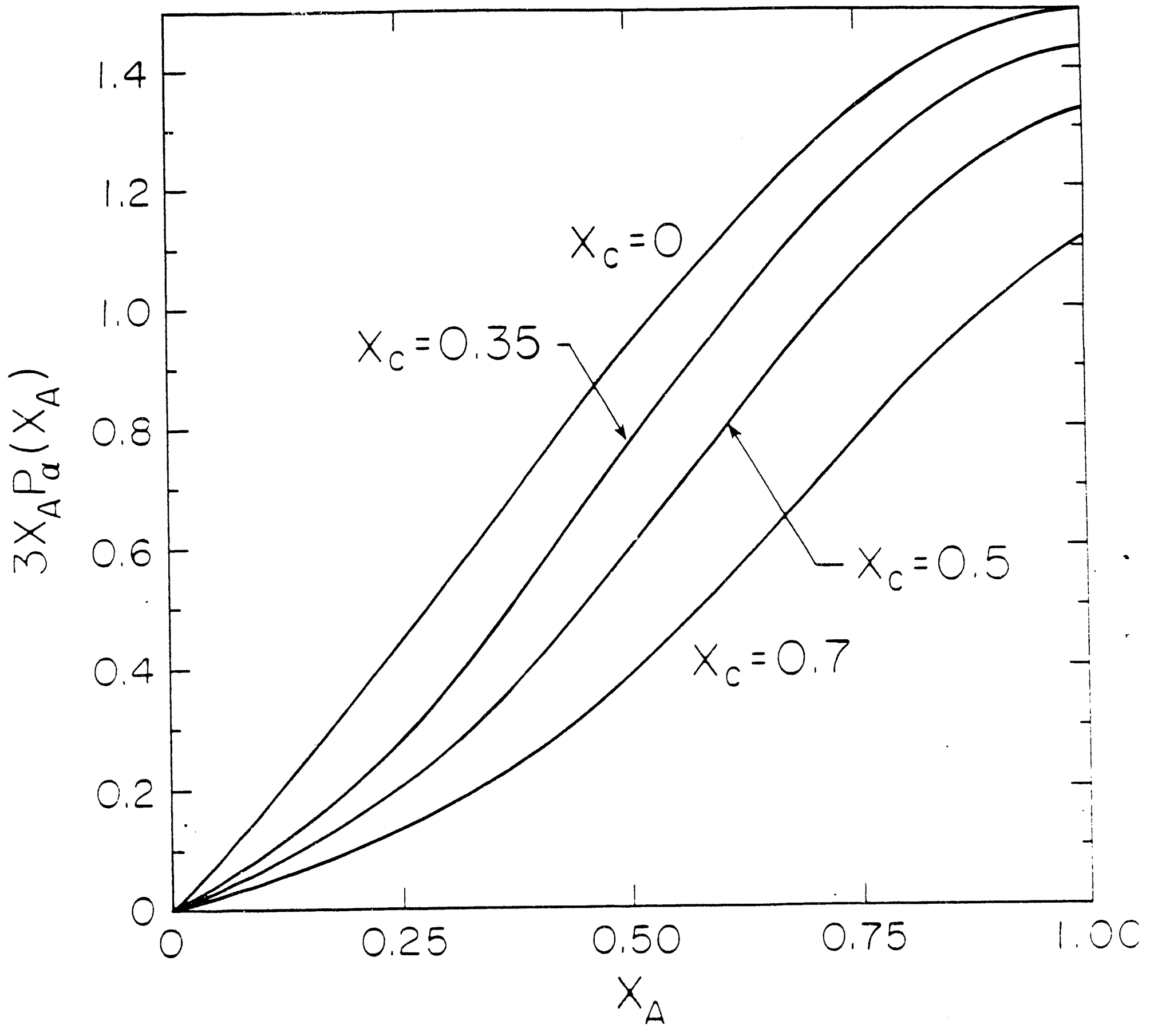

Fig. 12a 


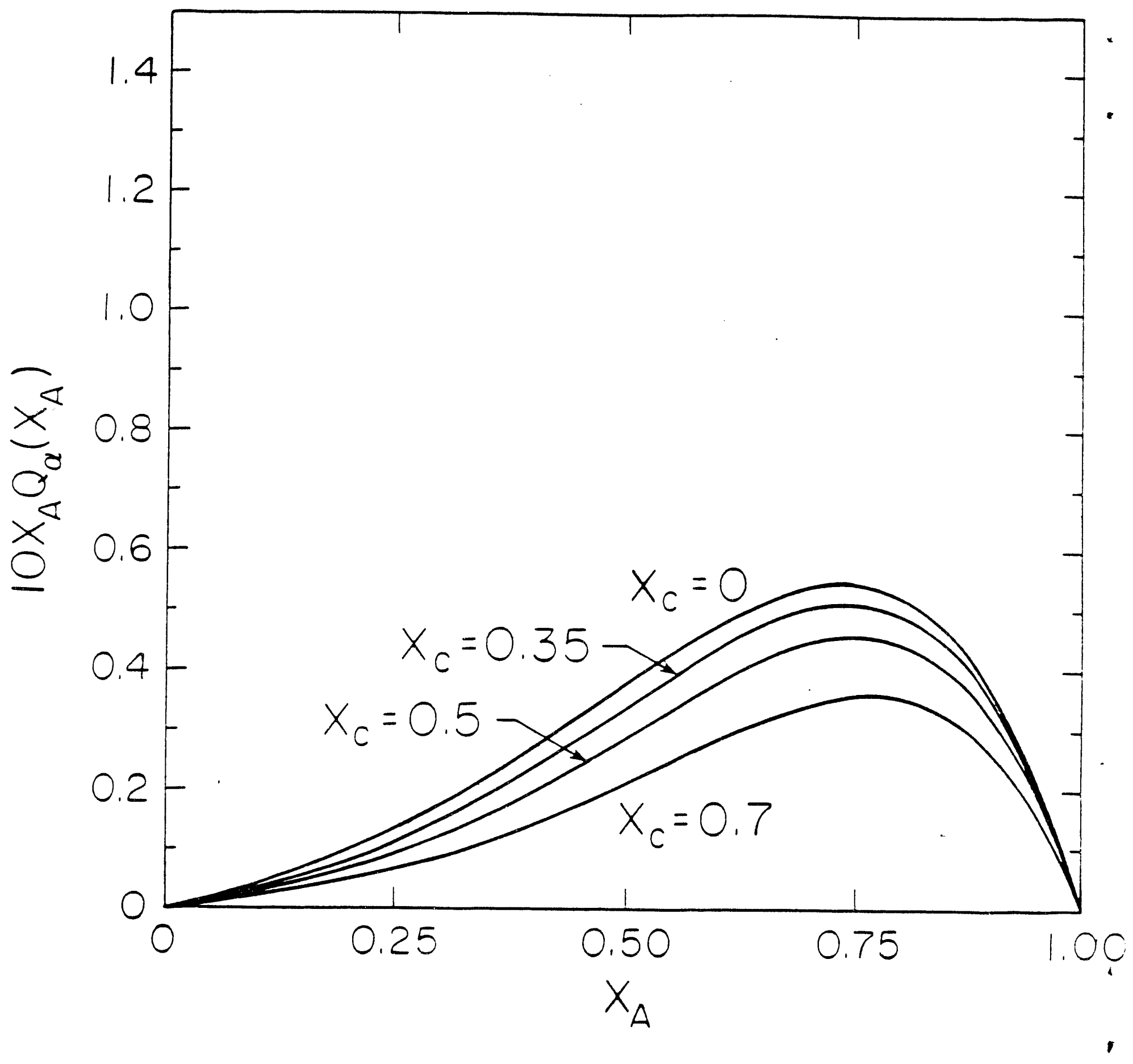




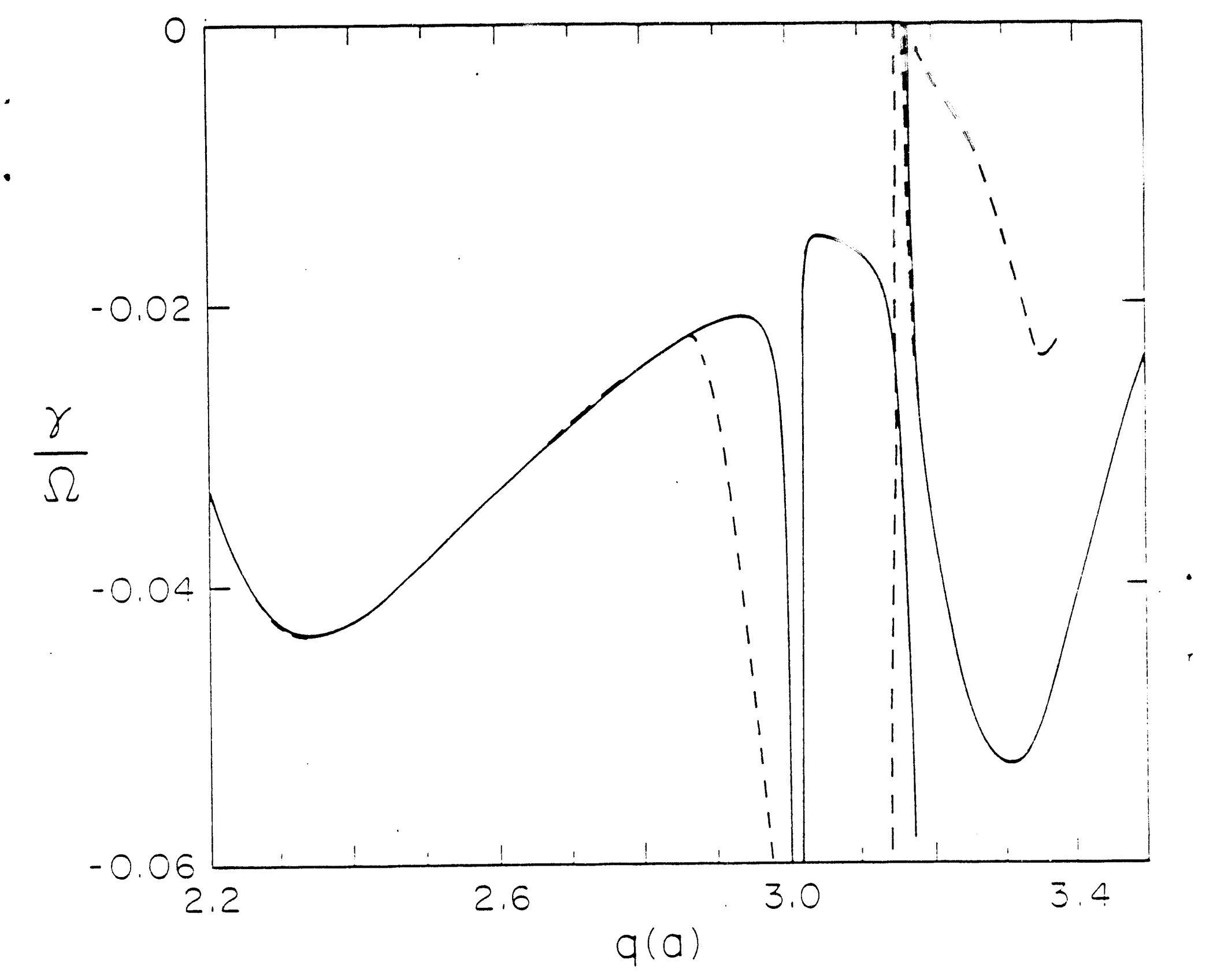

Fig. 13 

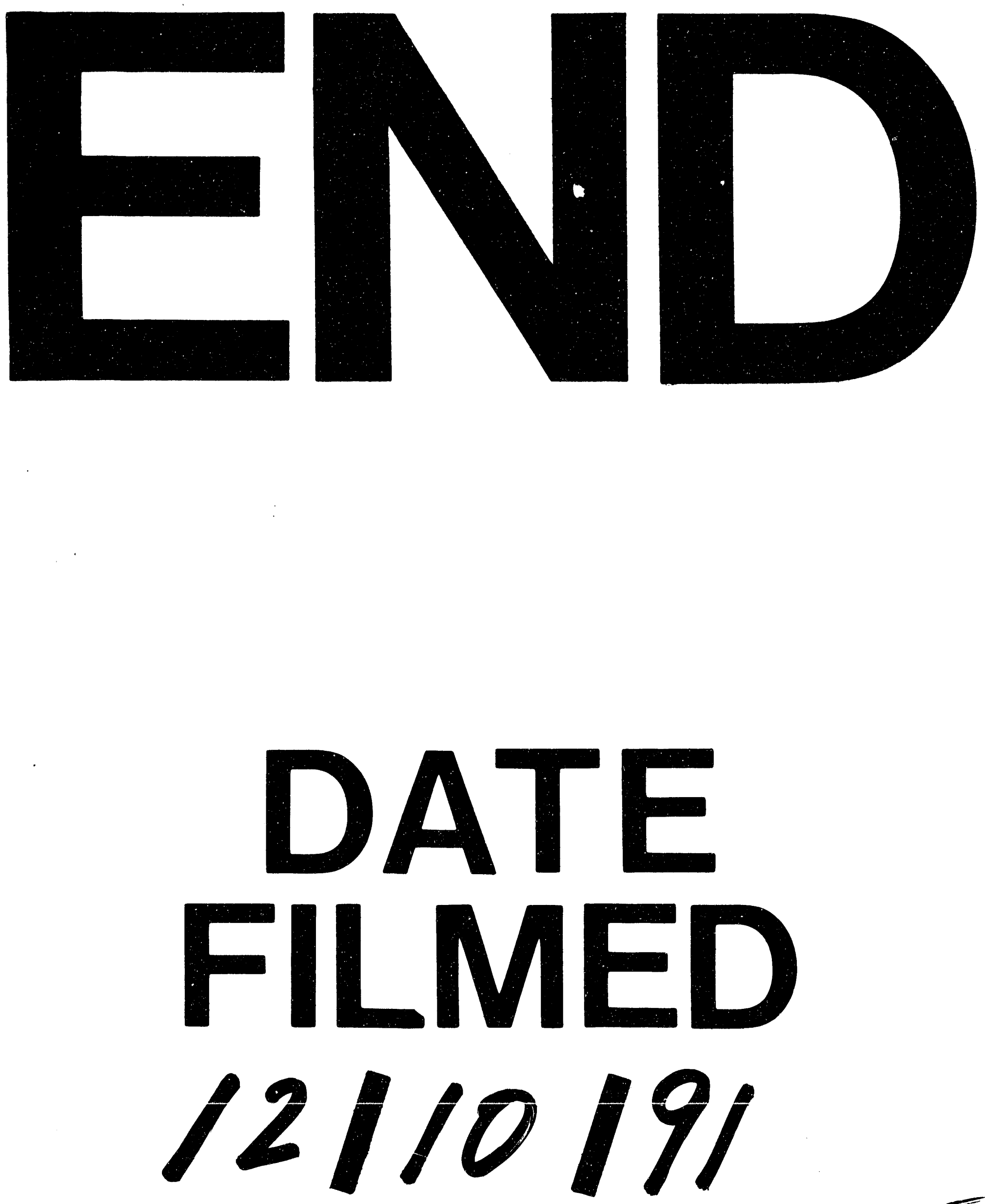
